Indium-mediated Asymmetric Barbier-type Allylation of Aldimines in Alcoholic Solvents: Synthesis of Optically Active Homoallylic Amines

Tirayut Vilaivan, ${ }^{* \dagger}$ Chutima Winotapan,,$^{\dagger}$ Vorawit Banphavichit, ${ }^{\dagger}$ Tetsuro Shinada ${ }^{\dagger}$ and Yasufumi Ohfune $e^{\ddagger}$

${ }^{\dagger}$ Organic Synthesis Research Unit, Department of Chemistry, Faculty of Science, Chulalongkorn University, Phayathai Road, Patumwan, Bangkok 10330, THAILAND.

${ }^{\ddagger}$ Department of Material Sciences, Graduate School of Science, Sugimoto, Sumiyoshi, Osaka City University, Osaka, JAPAN

\title{
Table of Content
}

\section{Page Description}

S2 Characetrization data for known compounds

S3 Figure S1: ${ }^{1} \mathrm{H}-\mathrm{NMR}$ spectrum of $(S)$ - and $(R)$-BPG derivatives of 1-phenylbut-3-enamine derived from $\mathbf{2 f}$ after oxidative cleavage by lead tetraacetate and $3 \mathrm{~N} \mathrm{HCl}$

S4 Figure S2: ${ }^{1} \mathrm{H}-\mathrm{NMR}$ spectrum of $(S)$ - and $(R)$-BPG derivatives of 1-phenylbut-3-enamine derived from $3 \mathbf{e}$ after oxidative cleavage by lead tetraacetate and $3 \mathrm{~N} \mathrm{HCl}$

S5 Figure S3: ${ }^{1} \mathrm{H}-\mathrm{NMR}$ spectrum of $(S)$ - and $(R)$-BPG derivatives of 1-phenylbut-3-enamine derived from $\mathbf{2 f}$ after oxidative cleavage by lead tetraacetate and $\mathrm{NH}_{2} \mathrm{OH} . \mathrm{HCl}$

S6 Figure S4: ${ }^{1} \mathrm{H}-\mathrm{NMR}$ spectrum of $(S)$ - and $(R)$-BPG derivatives of 1isopropyl-but-3-enamine derived from $2 \mathrm{~s}$ after oxidative cleavage by lead tetraacetate and $3 \mathrm{~N} \mathrm{HCl}$

S7 Figure S5: ${ }^{1} \mathrm{H}-\mathrm{NMR}$ spectrum of $(S)$ - and $(R)$-BPG derivatives of 1 isopropyl-but-3-enamine derived from $2 \mathrm{~s}$ after oxidative cleavage by lead tetraacetate and $\mathrm{NH}_{2} \mathrm{OH} . \mathrm{HCl}$

S8 Figure S6: $400 \mathrm{MHz}$ 1H-NMR spectra of a solution obtained from treatment of indium powder with excess allyl bromide in $\mathrm{CD}_{3} \mathrm{OD}$ before (bottom) and after (top) addition of excess $\mathrm{N}$-benzylidenebenzylamine.

S9-S92 ${ }^{1} \mathrm{H}$ and ${ }^{13} \mathrm{C}$ NMR spectra of compounds $\mathbf{2 e - 2 z}, \mathbf{4 e - 4 z}$ 


\section{Characetrization data for known compounds}

(2S)-2-phenyl-2-[(1'S)-1'-phenylbut-3'-enylamino]ethanol (2e)

(Agami, C.; Couty, F.; Evano, G. Tetrahedron: Asymmetry 2000, 11, 4639-4643)

colourless oil; $[\alpha]_{\mathrm{D}}^{24}=+43.0\left(c=1.01, \mathrm{CHCl}_{3}\right),{ }^{1} \mathrm{H}$ NMR $\left(400 \mathrm{MHz}, \mathrm{CDCl}_{3}\right) \delta 2.50-2.65$ (m, 2H), 3.59 (dd, $J=10.5,9.0 \mathrm{~Hz}, 1 \mathrm{H}), 3.80$ (m, 2H), 3.92 (dd, $J=7.0,4.5 \mathrm{~Hz}, 1 \mathrm{H}), 5.10$ $(\mathrm{m}, 2 \mathrm{H}), 5.82(\mathrm{~m}, 1 \mathrm{H}), 7.25-7.40(\mathrm{~m}, 10 \mathrm{H}) ;{ }^{13} \mathrm{C} \mathrm{NMR}\left(100 \mathrm{MHz}, \mathrm{CDCl}_{3}\right) \delta 41.3,59.8$, 61.4, 65.6, 117.5, 127.2, 127.3, 127.5, 128.4, 128.6, 135.0, 141.2, 143.6; HRMS (CI+) Calcd for $\mathrm{C}_{18} \mathrm{H}_{21} \mathrm{NO} \cdot \mathrm{H}^{+} 268.1701$, Found 268.1683 .

(2R)-2-phenyl-2-[(1'R)-1'-phenylbut-3'-enylamino]ethanol (2f)

(Yanada, R.; Kaieda, A.; Takemoto, Y. J. Org. Chem. 2001, 66, 7516-7518)

colourless oil; $[\alpha]^{23}{ }_{\mathrm{D}}=-42.0\left(c=1.05, \mathrm{CHCl}_{3}\right),\left(\right.$ lit. $\left.[\alpha]_{\mathrm{D}}^{29}=-42.3\left(c=4.00, \mathrm{CHCl}_{3}\right)\right) .{ }^{1} \mathrm{H}$ NMR $\left(400 \mathrm{MHz}, \mathrm{CDCl}_{3}\right) \delta 2.50-2.75(\mathrm{~m}, 2 \mathrm{H}), 3.60(\mathrm{dd}, J=10.7,9.0 \mathrm{~Hz}, 1 \mathrm{H}), 3.80(\mathrm{~m}$, $2 \mathrm{H}), 3.93(\mathrm{dd}, J=6.9,4.6 \mathrm{~Hz}, 1 \mathrm{H}), 5.10(\mathrm{~m}, 1 \mathrm{H}), 5.78(\mathrm{~m}, 1 \mathrm{H}), 7.24-7.40(\mathrm{~m}, 10 \mathrm{H}) ;{ }^{13} \mathrm{C}$ NMR $\left(100 \mathrm{MHz}, \mathrm{CDCl}_{3}\right) \delta 41.0,59.8,61.4,65.5,117.5,127.2,127.4,127.5,128.4$, 128.6, 134.9, 141.1, 143.5; MS (ESI+) $m / z 268.2(\mathrm{M} \cdot \mathrm{H})^{+}$; Anal. Calcd for $\mathrm{C}_{18} \mathrm{H}_{21} \mathrm{NO}$ : C, 80.86; H, 7.92; N, 5.24. Found: C, 80.86; H, 7.98; N, $5.23 \%$

(2R)-2-phenyl-2-[(1'R)-1'-isopropylbut-3'-enylamino]ethanol (2s). (Agami, C.; Couty, F.; Evano, G. Tetrahedron: Asymmetry 2000, 11, 4639-4643) Yellow solid: m.p. $52-53{ }^{\circ} \mathrm{C} ;{ }^{1} \mathrm{H}$ NMR $\left(400 \mathrm{MHz}, \mathrm{CDCl}_{3}\right) \delta 0.82$ and $0.84(2 \times \mathrm{d}, J=6.8$ $\mathrm{Hz}, 6 \mathrm{H}), 1.63(\mathrm{~m}, 1 \mathrm{H}), 2.20(\mathrm{~m}, 2 \mathrm{H}), 2.32(\mathrm{~m}, 1 \mathrm{H}), 3.50(\mathrm{dd}, J=10.5,8.5 \mathrm{~Hz}, 1 \mathrm{H}), 3.68$ (dd, $J=10.6,4.5 \mathrm{~Hz}, 1 \mathrm{H}), 3.92$ (dd, $J=9.0,4.5 \mathrm{~Hz}, 1 \mathrm{H}), 5.05(\mathrm{~m}, 2 \mathrm{H}), 5.82(\mathrm{~m}, 1 \mathrm{H}), 7.32$ $(\mathrm{m}, 5 \mathrm{H}) ;{ }^{13} \mathrm{C} \mathrm{NMR}\left(100 \mathrm{MHz}, \mathrm{CDCl}_{3}\right) \delta 18.2,18.8,30.5,35.1,59.5,61.9,66.9,116.8$, $127.4,127.5,128.5,136.1,141.4$; MS (ESI+) $m / z 234.2(\mathrm{M} \cdot \mathrm{H})^{+}$; Anal. Calcd for $\mathrm{C}_{15} \mathrm{H}_{23} \mathrm{NO}$ : C, 77.21; H, 9.93; N, 6.00. Found: C, 77.03; H, 9.90; N, $5.95 \%$

(2R)-2-phenyl-2-[(1'R)-1'-(2"'-phenylethenyl)but-3'-enylamino]ethanol (2y) (Allin, S.M.; Button, M.A.C.; Baird, R.D. Synlett 1998, 1117-1119.) yellow oil; $[\alpha]^{24}{ }_{\mathrm{D}}=+26.0\left(c=1.00, \mathrm{CHCl}_{3}\right) ;{ }^{1} \mathrm{H} \mathrm{NMR}\left(400 \mathrm{MHz}, \mathrm{CDCl}_{3}\right) \delta 2.42(2 \mathrm{H}, \mathrm{m}$, $\mathrm{C}_{2}$ allyl), 2.60 (br m, 1H), 3.40 (dd, $\left.J=13.9,5.8 \mathrm{~Hz}, 1 \mathrm{H}\right), 3.62$ (dd, $\left.J=10.7,7.6 \mathrm{~Hz}, 1 \mathrm{H}\right)$, 3.80 (dd, $J=10.8,4.6 \mathrm{~Hz}, 1 \mathrm{H}), 4.00$ (dd, $J=7.4,4.5 \mathrm{~Hz}, 1 \mathrm{H}), 5.10$ (d, $J=10.1 \mathrm{~Hz}, 2 \mathrm{H}), 5.92$ $(\mathrm{m}, 1 \mathrm{H}), 6.04(\mathrm{dd}, J=16.0,8.0 \mathrm{~Hz}, 1 \mathrm{H}), 6.48(\mathrm{~d}, J=15.9 \mathrm{~Hz}, 1 \mathrm{H}), 7.25-7.42(\mathrm{~m}, 10 \mathrm{H}) ;{ }^{13} \mathrm{C}$ NMR $\left(100 \mathrm{MHz}, \mathrm{CDCl}_{3}\right) \delta 39.8,58.1,61.5,66.0,117.7,126.3,127.3,127.4,127.5$, 128.6, 128.7, 130.6, 132.7, 135.0, 136.9, 141.6; HRMS (CI+) Calcd for $\mathrm{C}_{20} \mathrm{H}_{23} \mathrm{NO} \cdot \mathrm{H}^{+}$ 294.1858, Found 294.1871 $(\mathrm{M} \cdot \mathrm{H})^{+}$

N-tert-butoxycarbonyl-(S)-1-phenylbut-3-enamine (4e). (Laschat, S.; Kunz, H. J. Org. Chem. 1991, 56, 5883-5889.) White solid: m.p. $76-77^{\circ} \mathrm{C} ;[\alpha]_{\mathrm{D}}{ }^{24}-43.0\left(c=1.00, \mathrm{CHCl}_{3}\right)\left(\right.$ lit. $[\alpha]^{22}{ }_{\mathrm{D}}=-48.4(c=1.00$, $\left.\mathrm{CHCl}_{3}\right)$ ); ${ }^{1} \mathrm{H} \mathrm{NMR}\left(400 \mathrm{MHz}, \mathrm{CDCl}_{3}\right) \delta 1.44(\mathrm{~s}, 9 \mathrm{H}), 2.54(\mathrm{~m}, 2 \mathrm{H}), 4.77(\mathrm{br} \mathrm{m}, 1 \mathrm{H}), 4.98$ (br d, $J=6.4 \mathrm{~Hz}, 1 \mathrm{H}), 5.11(\mathrm{~m}, 2 \mathrm{H}), 5.70(\mathrm{~m}, 1 \mathrm{H}), 7.24-7.36(\mathrm{~m}, 5 \mathrm{H}) ;{ }^{13} \mathrm{C}$ NMR $(100$ $\left.\mathrm{MHz}, \mathrm{CDCl}_{3}\right) \delta 28.4,41.3,54.0,79.4,118.2,126.3,127.1,128.5,134.0,142.4,155.2$; HRMS (FAB+) Calcd for $\mathrm{C}_{15} \mathrm{H}_{21} \mathrm{O}_{2} \mathrm{~N} \cdot \mathrm{H}^{+} \mathrm{m} / \mathrm{z} 248.1650$, Found 284.1661. 

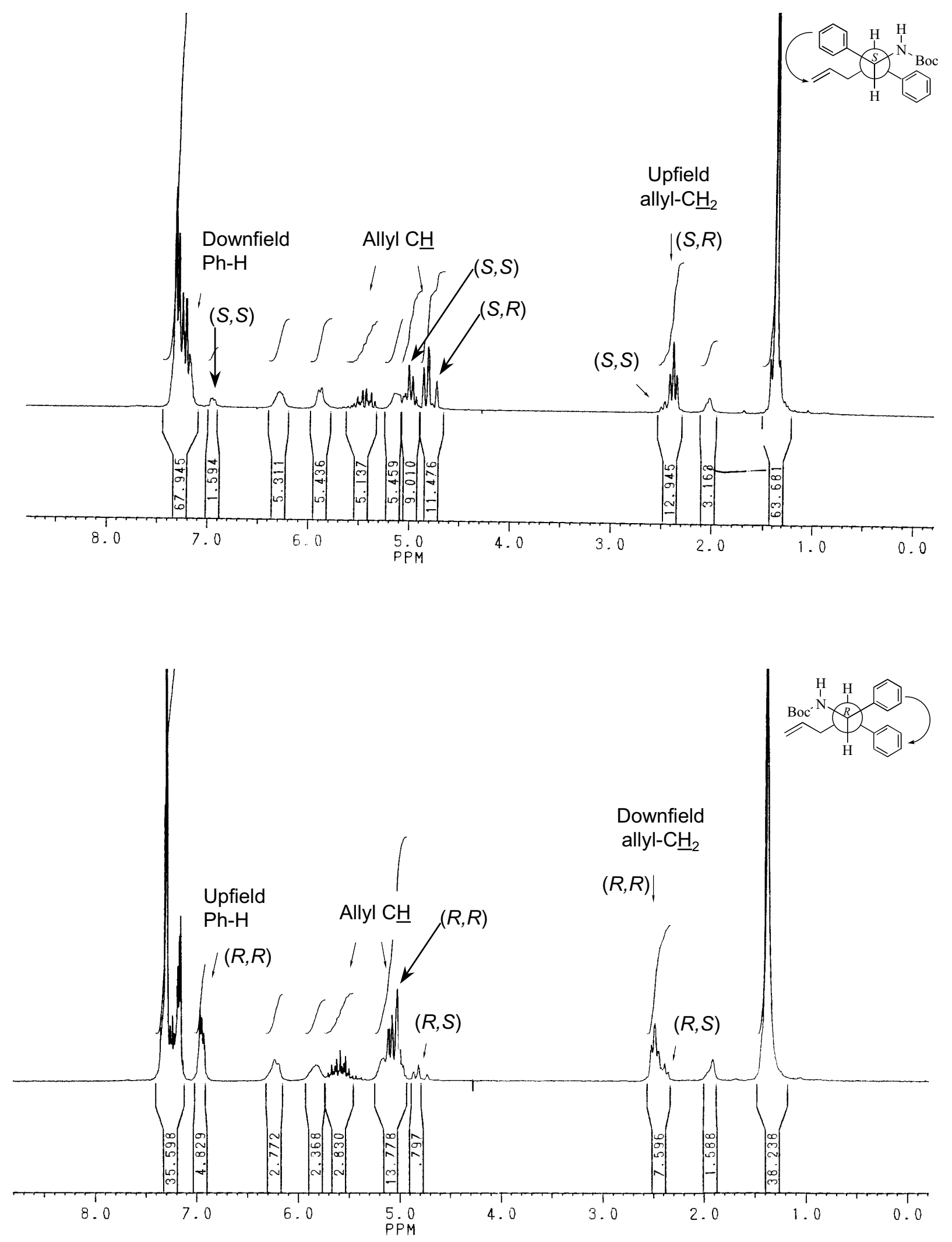

\section{Figure S1}

${ }^{1} \mathrm{H}-\mathrm{NMR}$ spectrum of $(S)$ - and $(R)$-BPG derivatives of 1-phenyl-but-3-enamine derived from $2 \mathbf{f}$ after oxidative cleavage by lead tetraacetate and $3 \mathrm{~N} \mathrm{HCl}$ 

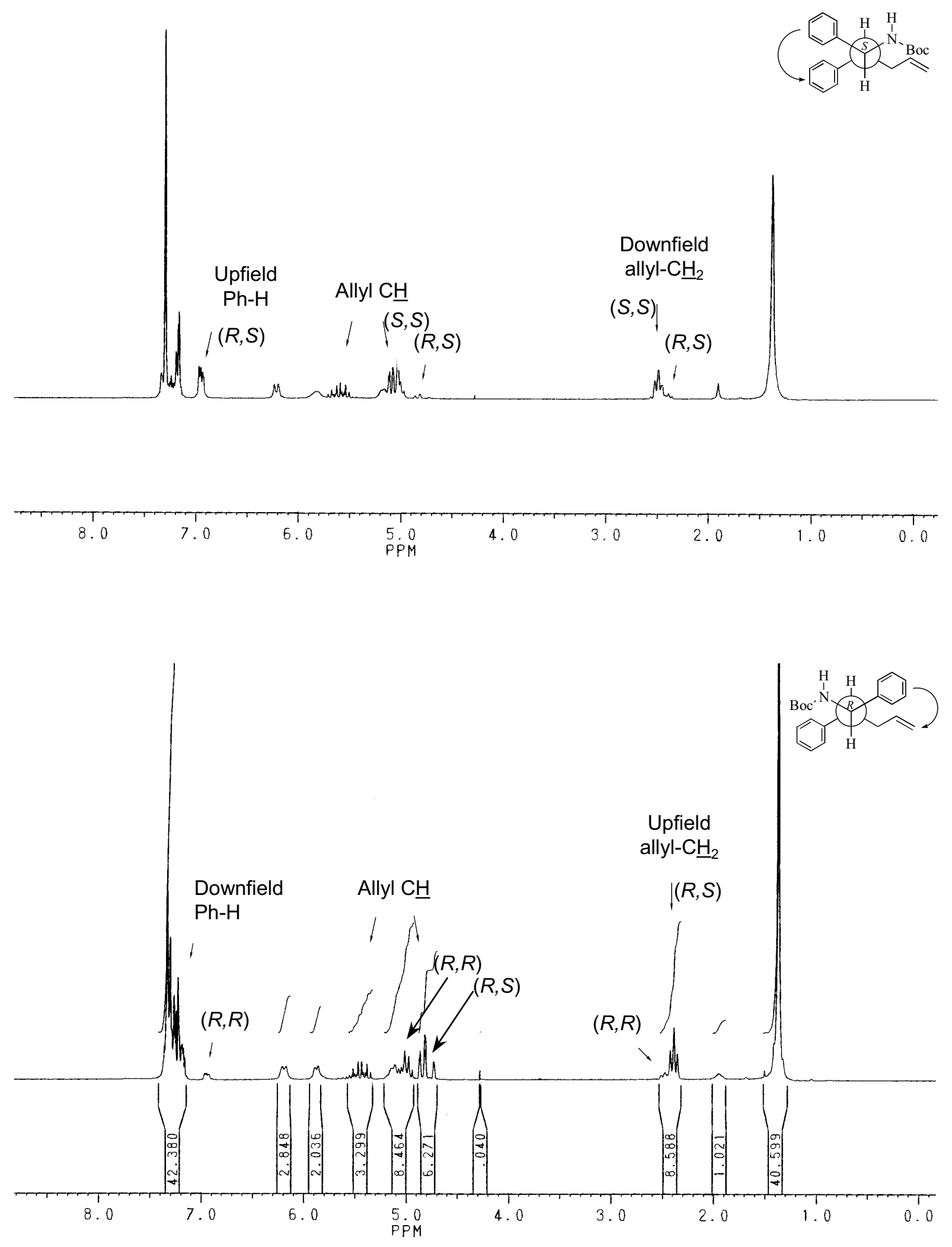

\section{Figure S2}

${ }^{1} \mathrm{H}-\mathrm{NMR}$ spectrum of $(S)$ - and $(R)$-BPG derivatives of 1-phenyl-but-3-enamine derived from $3 \mathbf{e}$ after oxidative cleavage by lead tetraacetate and $3 \mathrm{~N} \mathrm{HCl}$ 

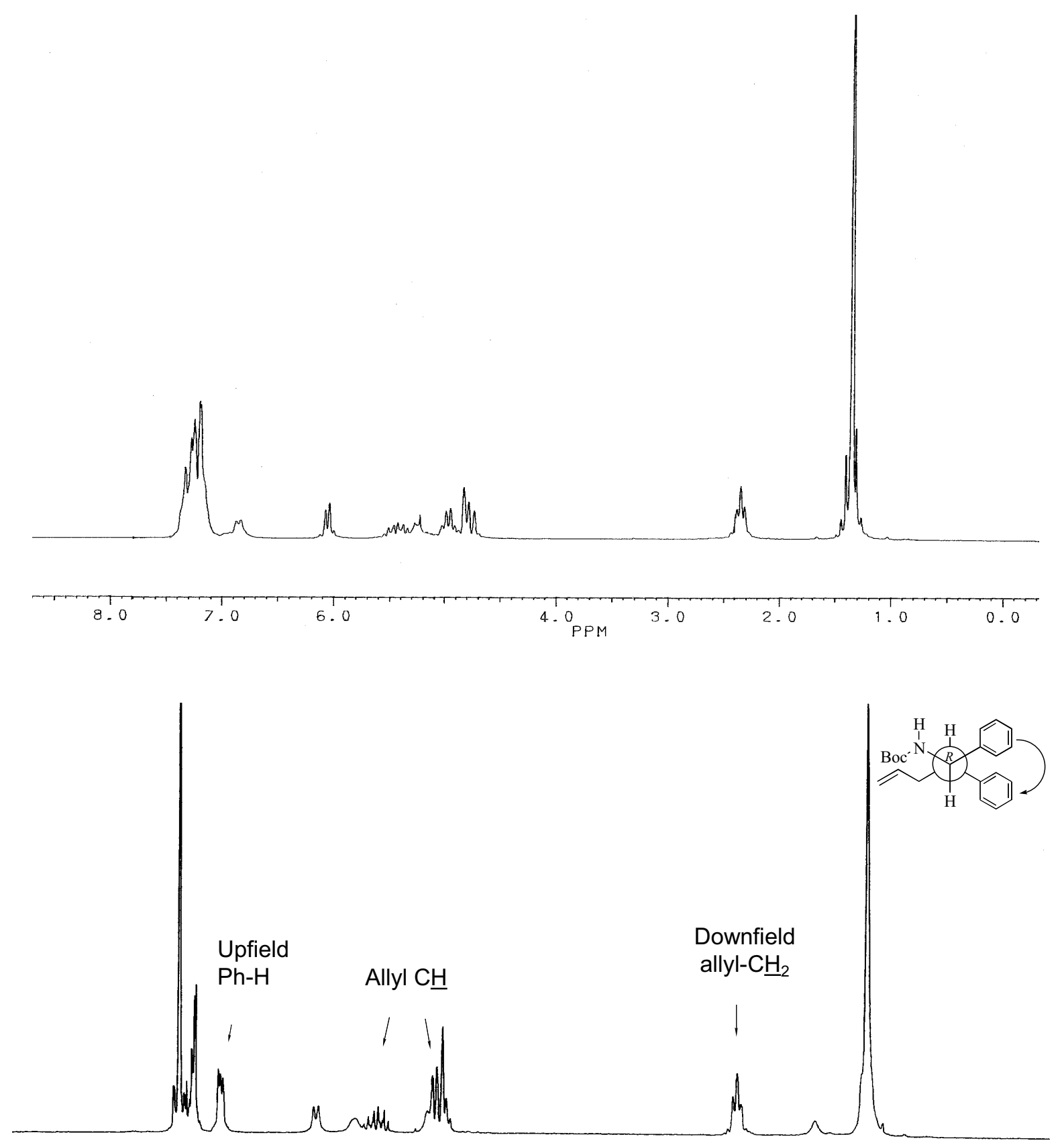

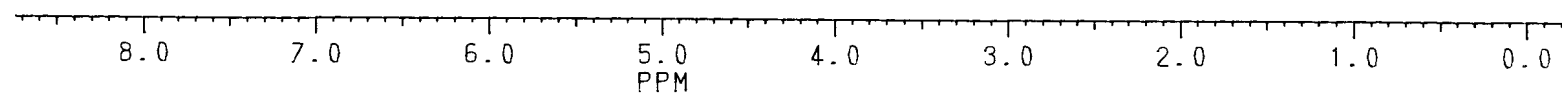

\section{Figure S3}

${ }^{1} \mathrm{H}-\mathrm{NMR}$ spectrum of $(S)$ - and $(R)$-BPG derivatives of 1-phenyl-but-3-enamine derived from $\mathbf{2 f}$ after oxidative cleavage by lead tetraacetate and $\mathrm{NH}_{2} \mathrm{OH} . \mathrm{HCl}$ 


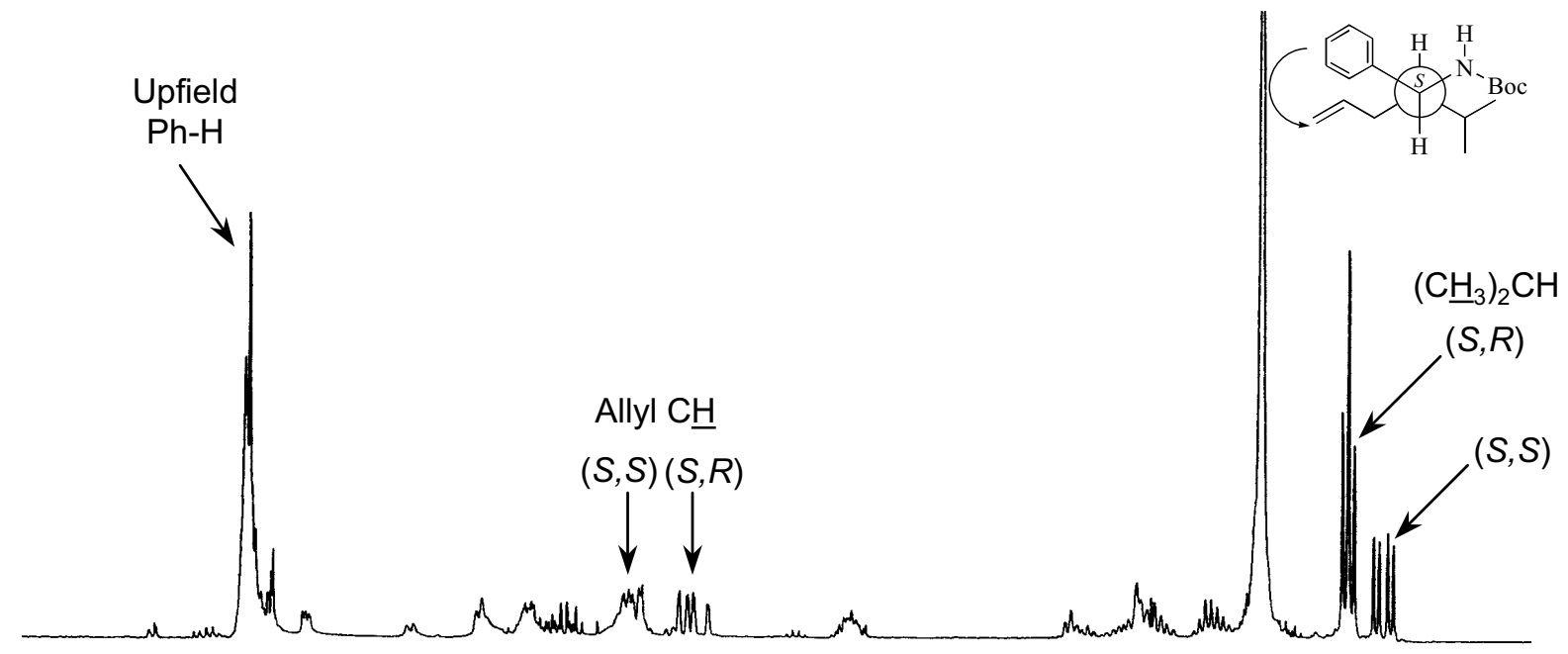

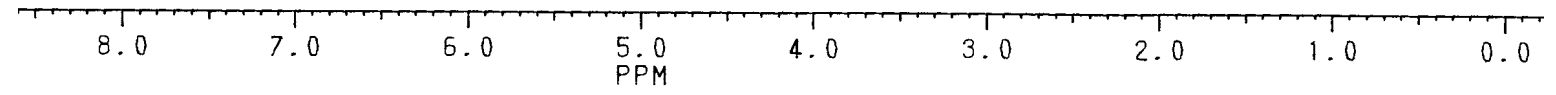
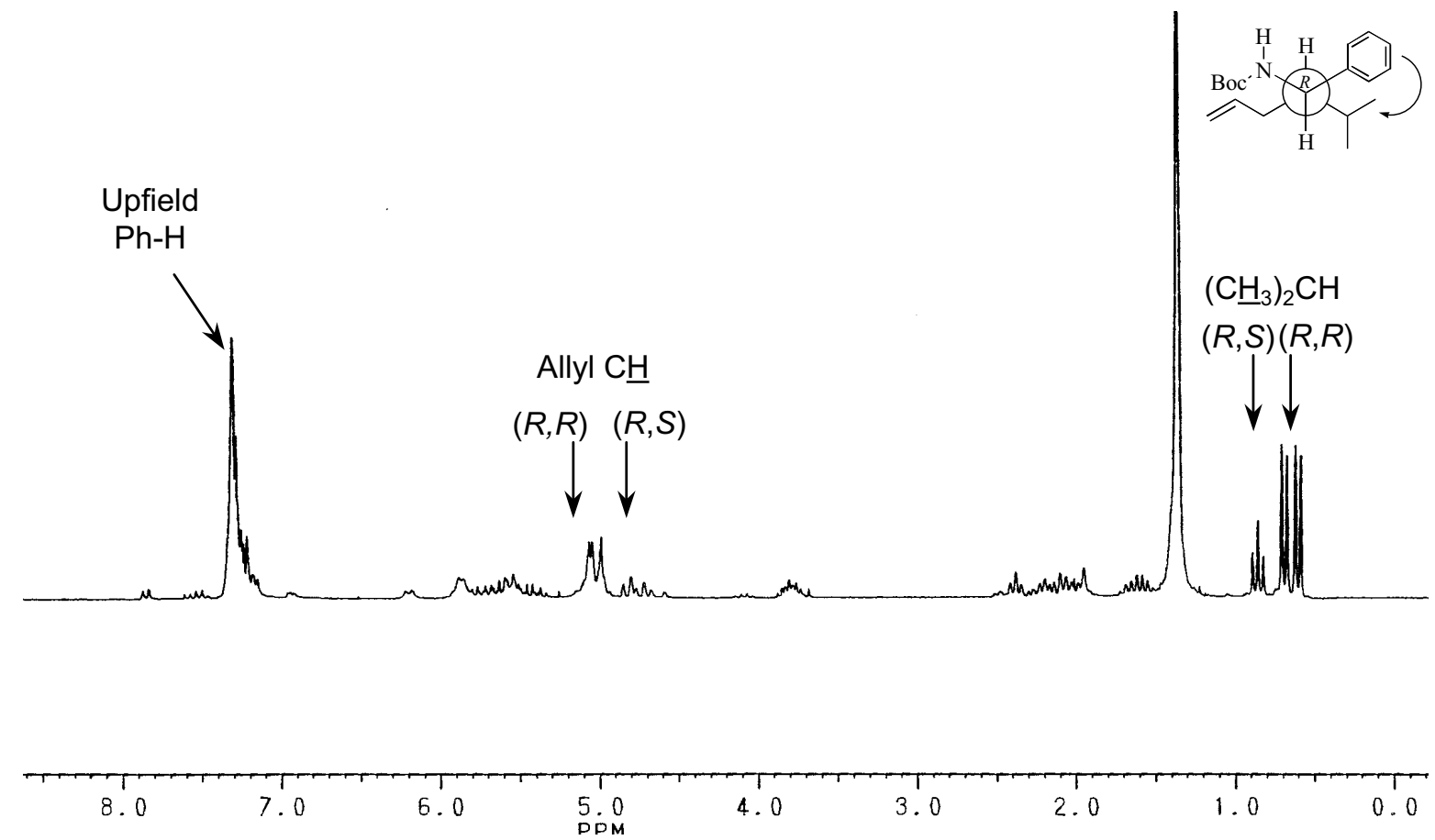

\section{Figure S4}

${ }^{1} \mathrm{H}$-NMR spectrum of $(S)$ - and $(R)$-BPG derivatives of 1-isopropyl-but-3-enamine derived from $2 \mathrm{~s}$ after oxidative cleavage by lead tetraacetate and $3 \mathrm{~N} \mathrm{HCl}$ 


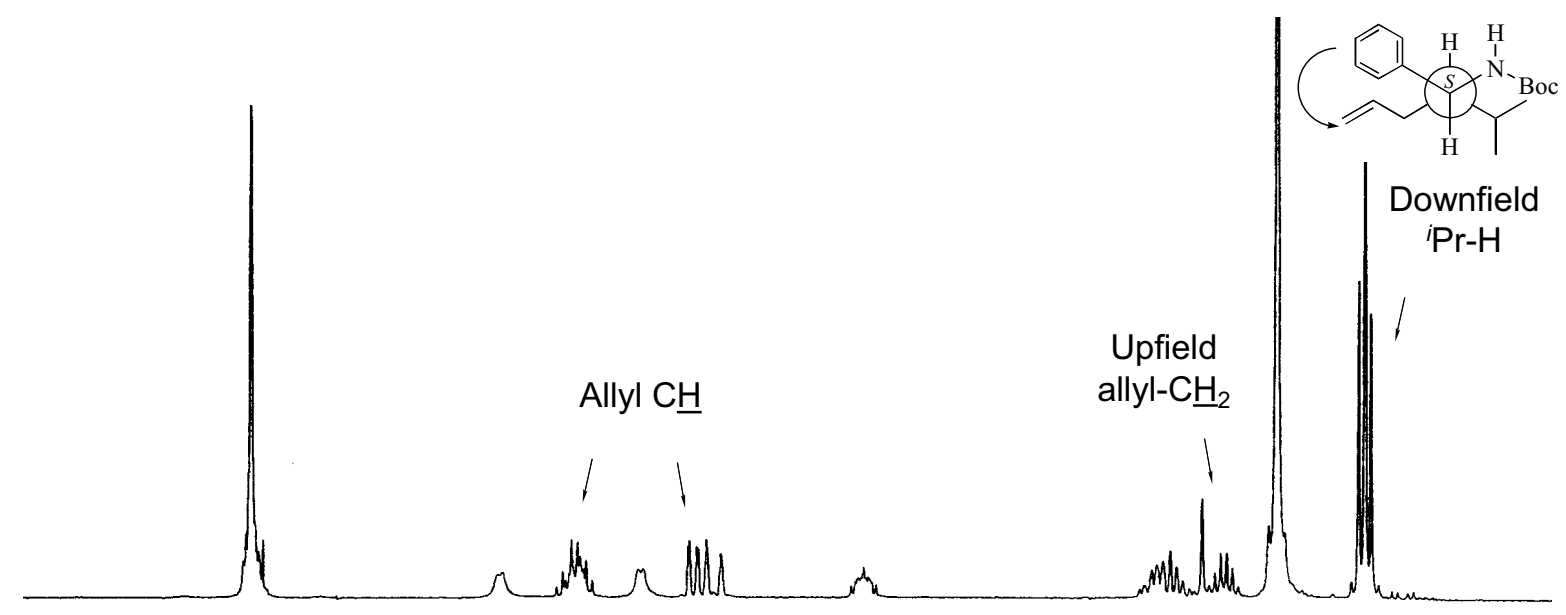

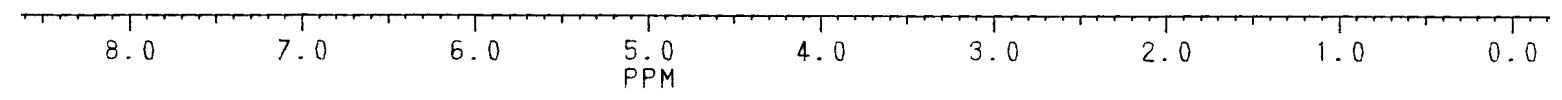

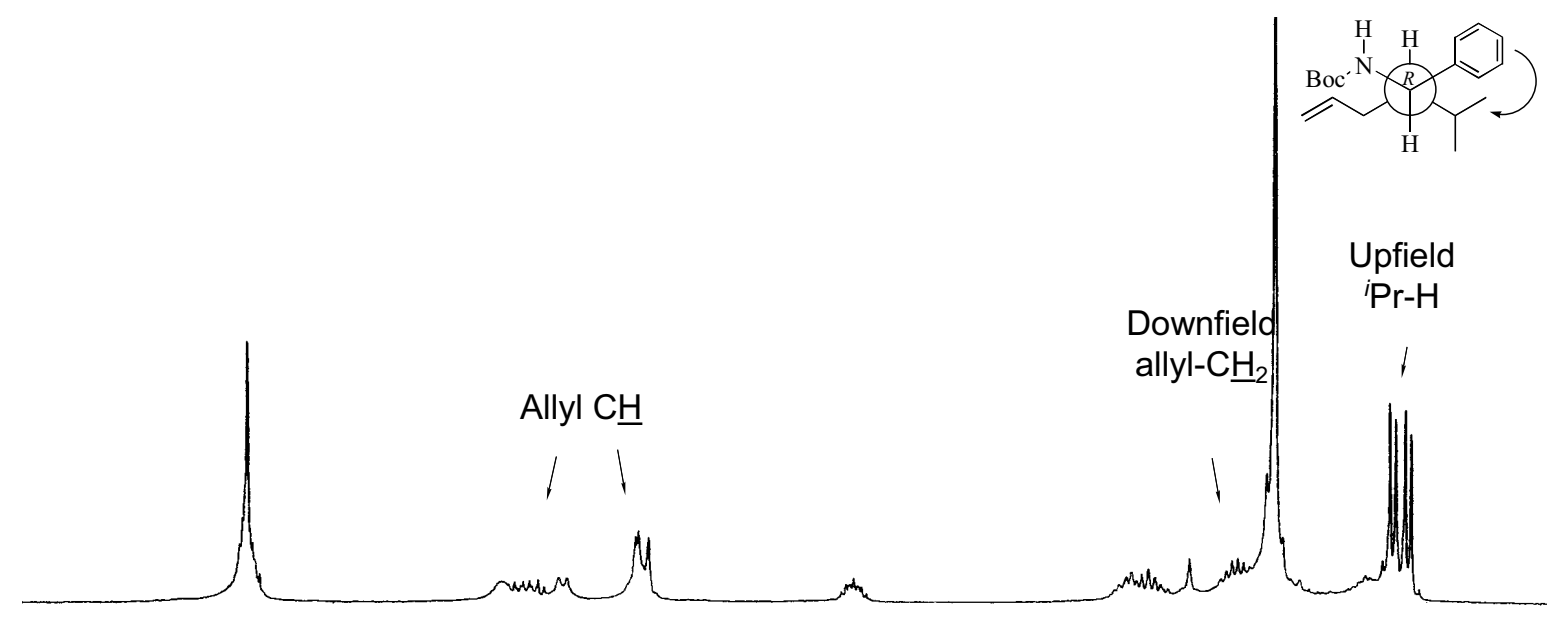

\begin{tabular}{|c|c|c|c|c|c|c|c|c|}
\hline 8.0 & 7.0 & 6.0 & 5.0 & 4.0 & 3.0 & 2.0 & 1.0 & 0.0 \\
\hline
\end{tabular}

\section{Figure S5}

${ }^{1} \mathrm{H}-\mathrm{NMR}$ spectrum of $(S)$ - and $(R)$-BPG derivatives of 1-isopropyl-but-3-enamine derived from $2 \mathrm{~s}$ after oxidative cleavage by lead tetraacetate and $\mathrm{NH}_{2} \mathrm{OH} . \mathrm{HCl}$ 

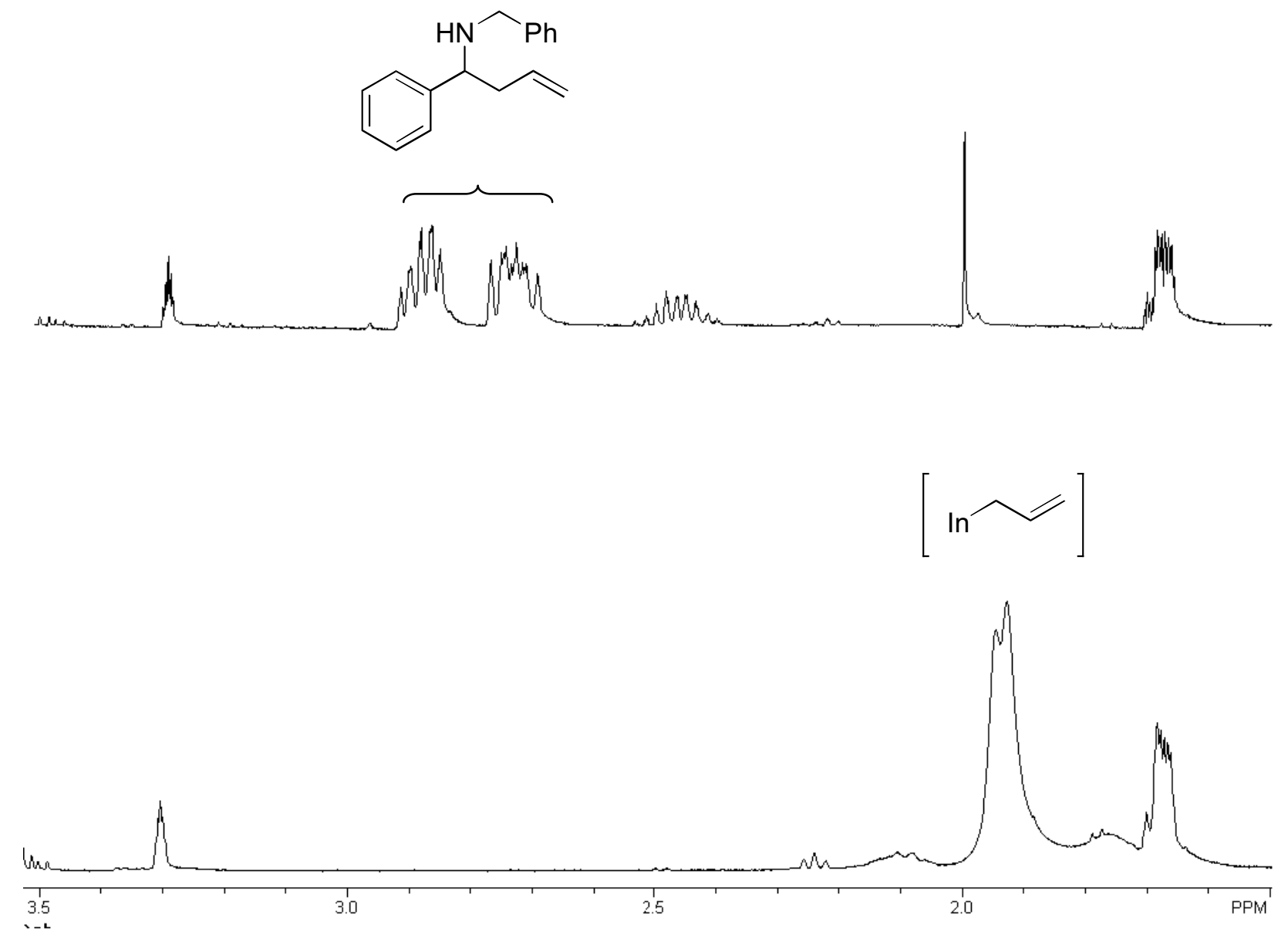

Figure S6

$400 \mathrm{MHz}{ }^{1} \mathrm{H}-\mathrm{NMR}$ spectra of a solution obtained from treatment of indium powder with excess allyl bromide in $\mathrm{CD}_{3} \mathrm{OD}$ before (bottom) and after (top) addition of excess $\mathrm{N}$-benzylidenebenzylamine. Only the allylic $\mathrm{CH}_{2}$ signals were shown. 

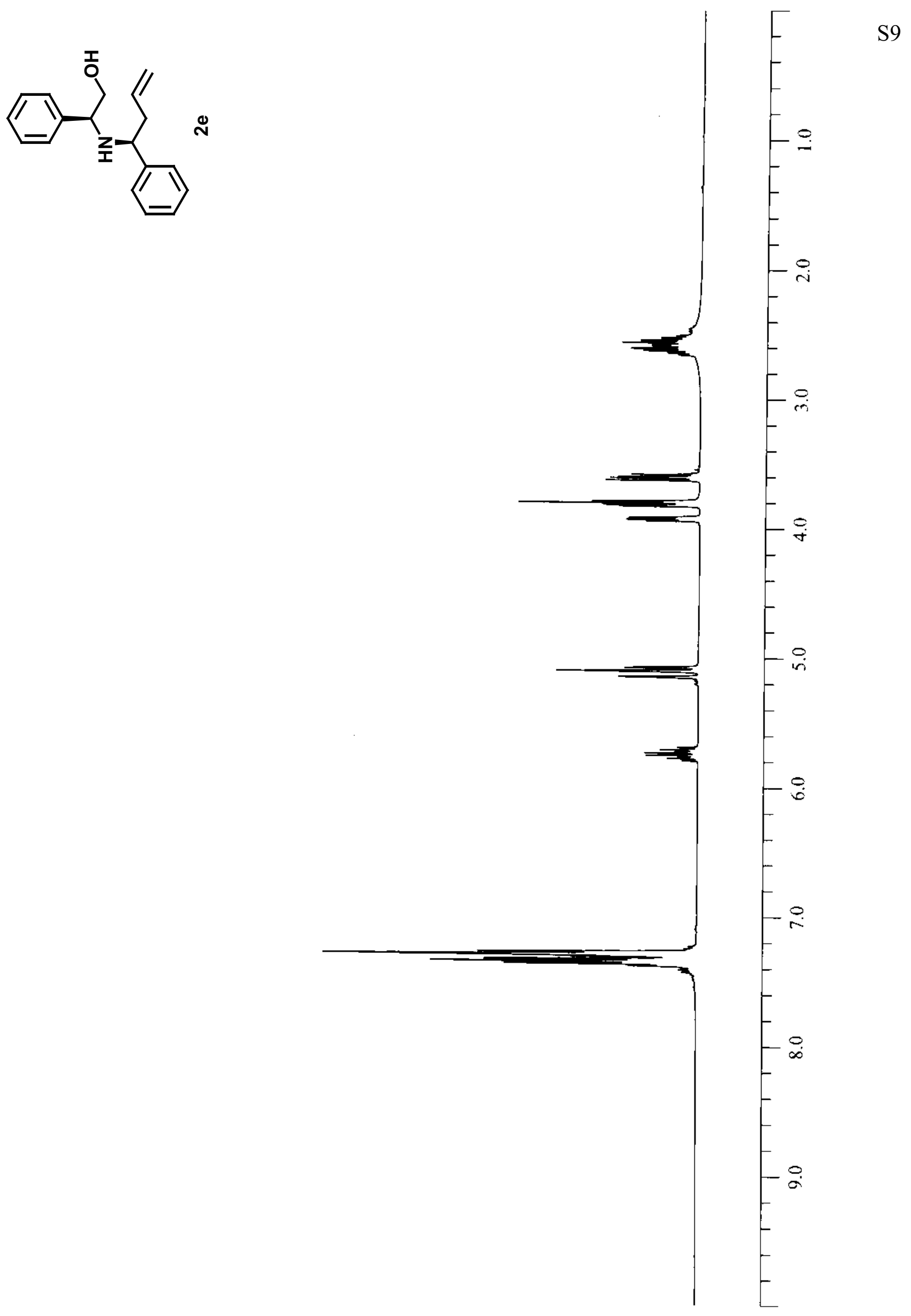

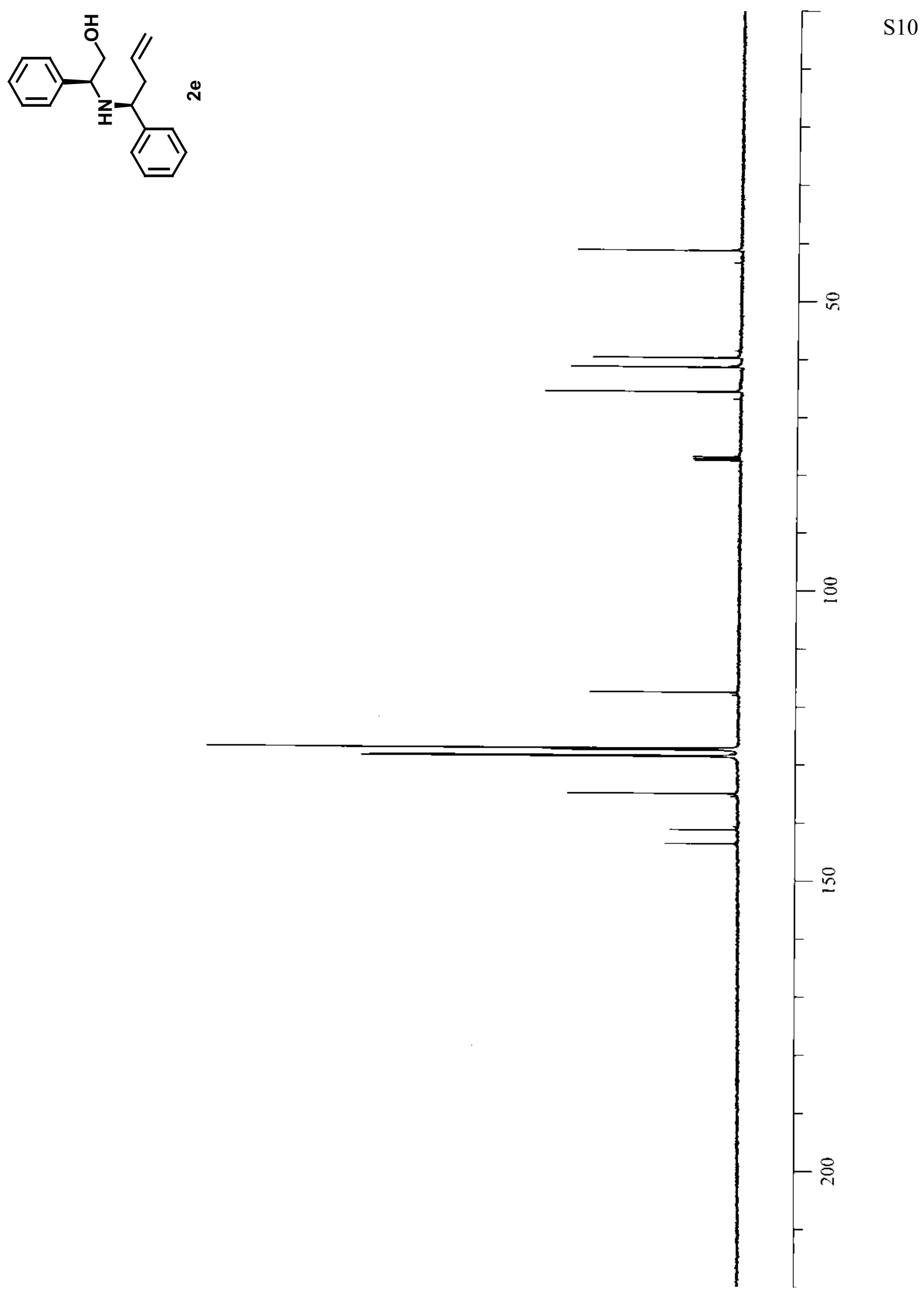

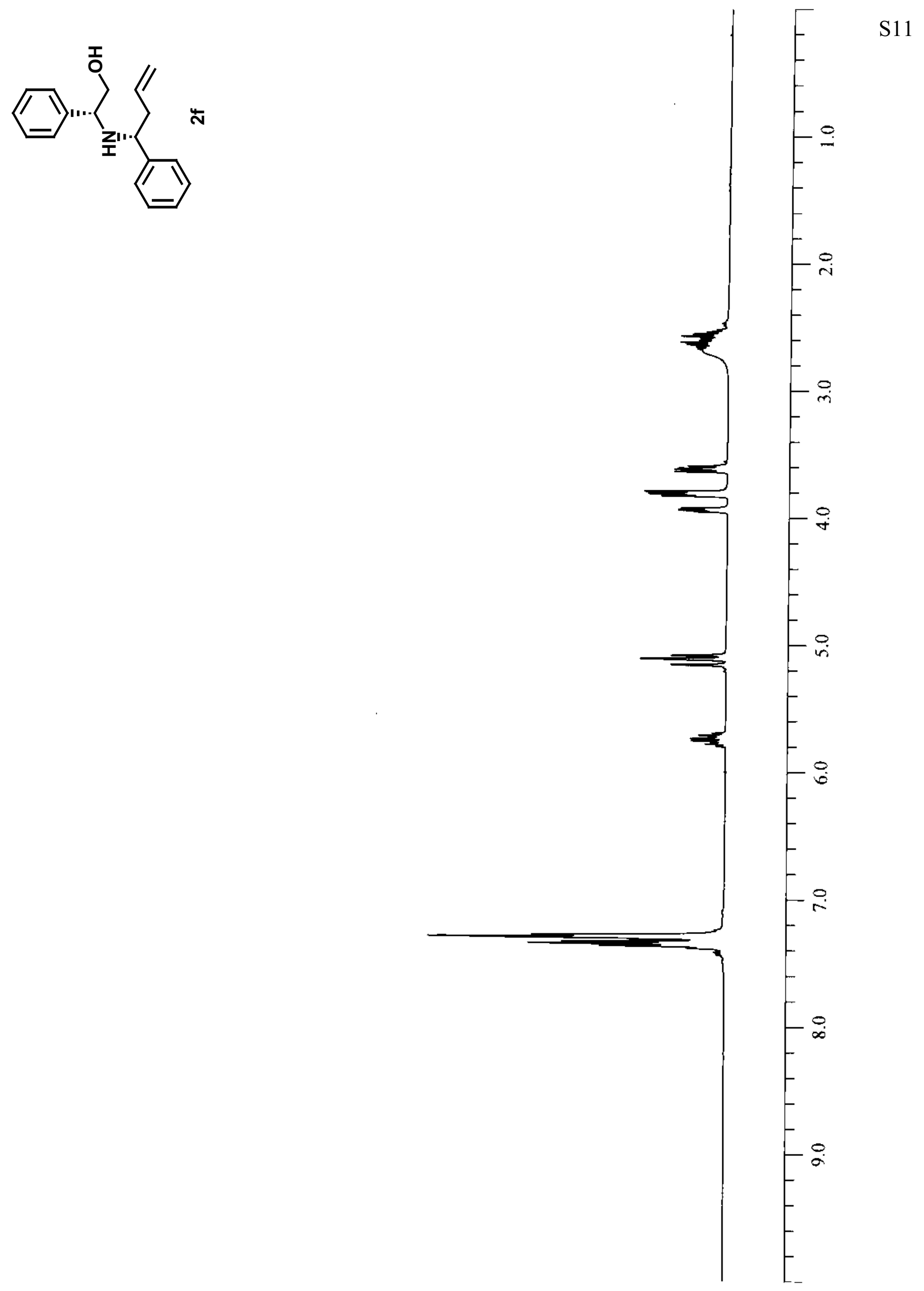

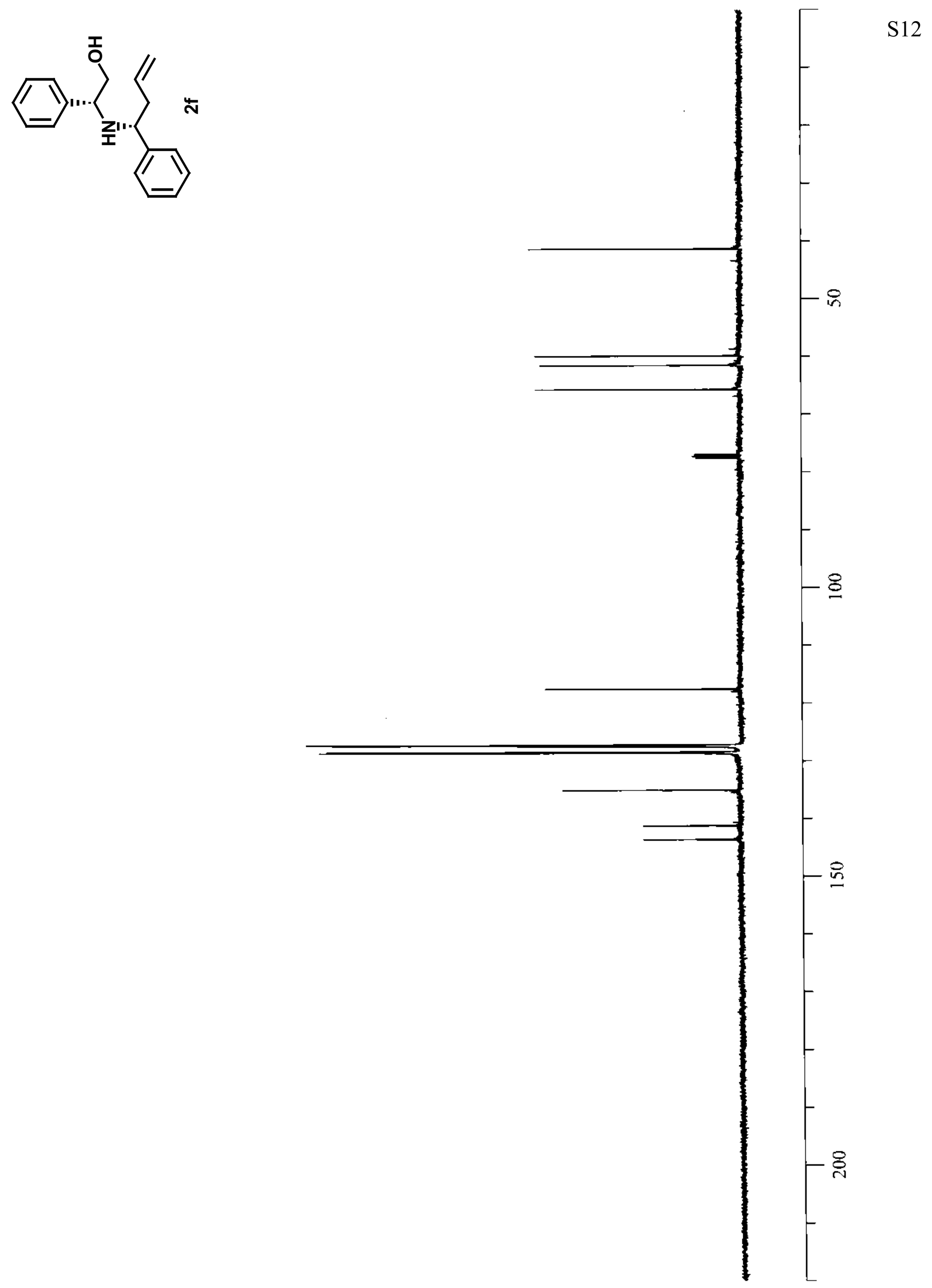

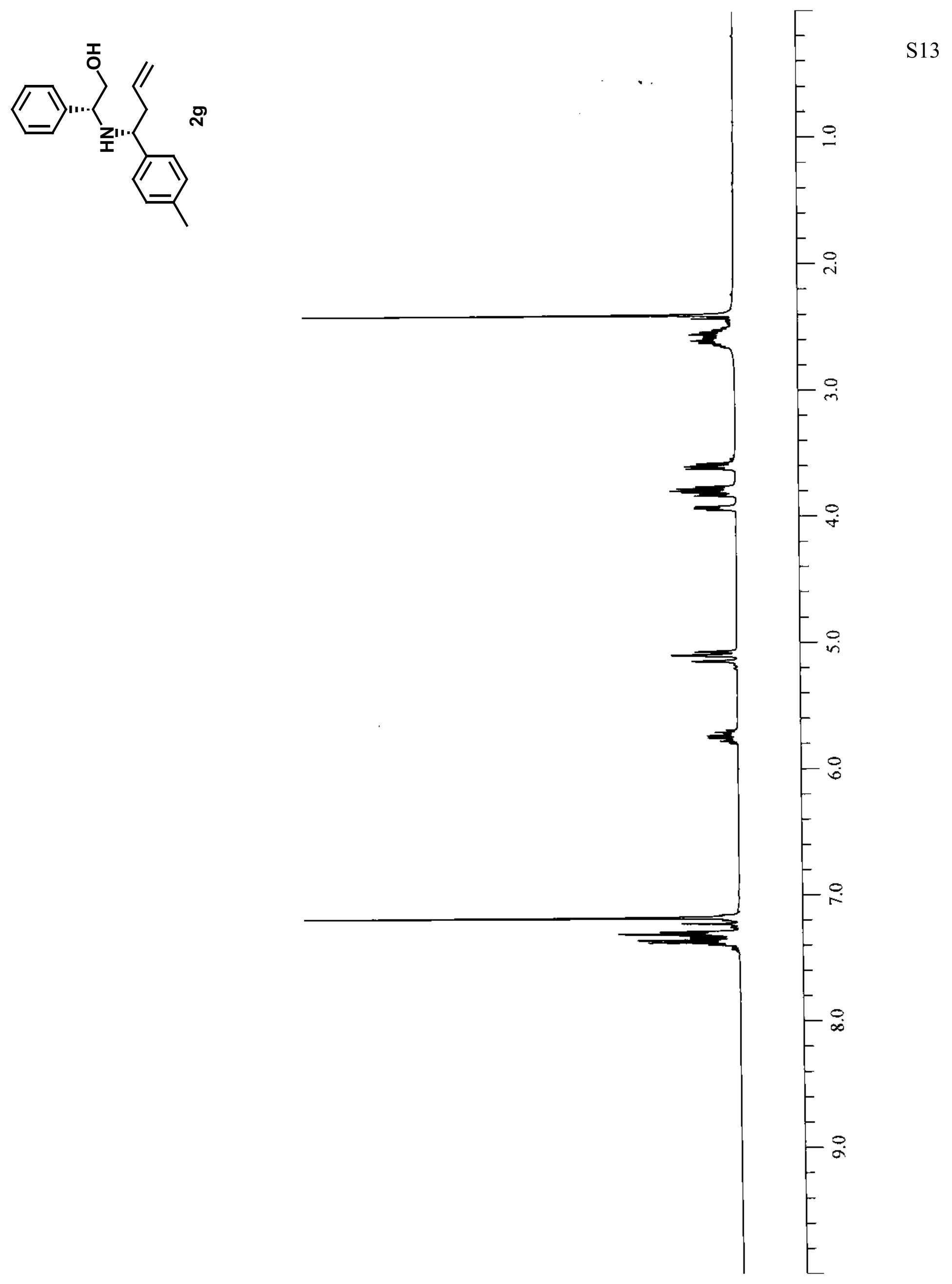

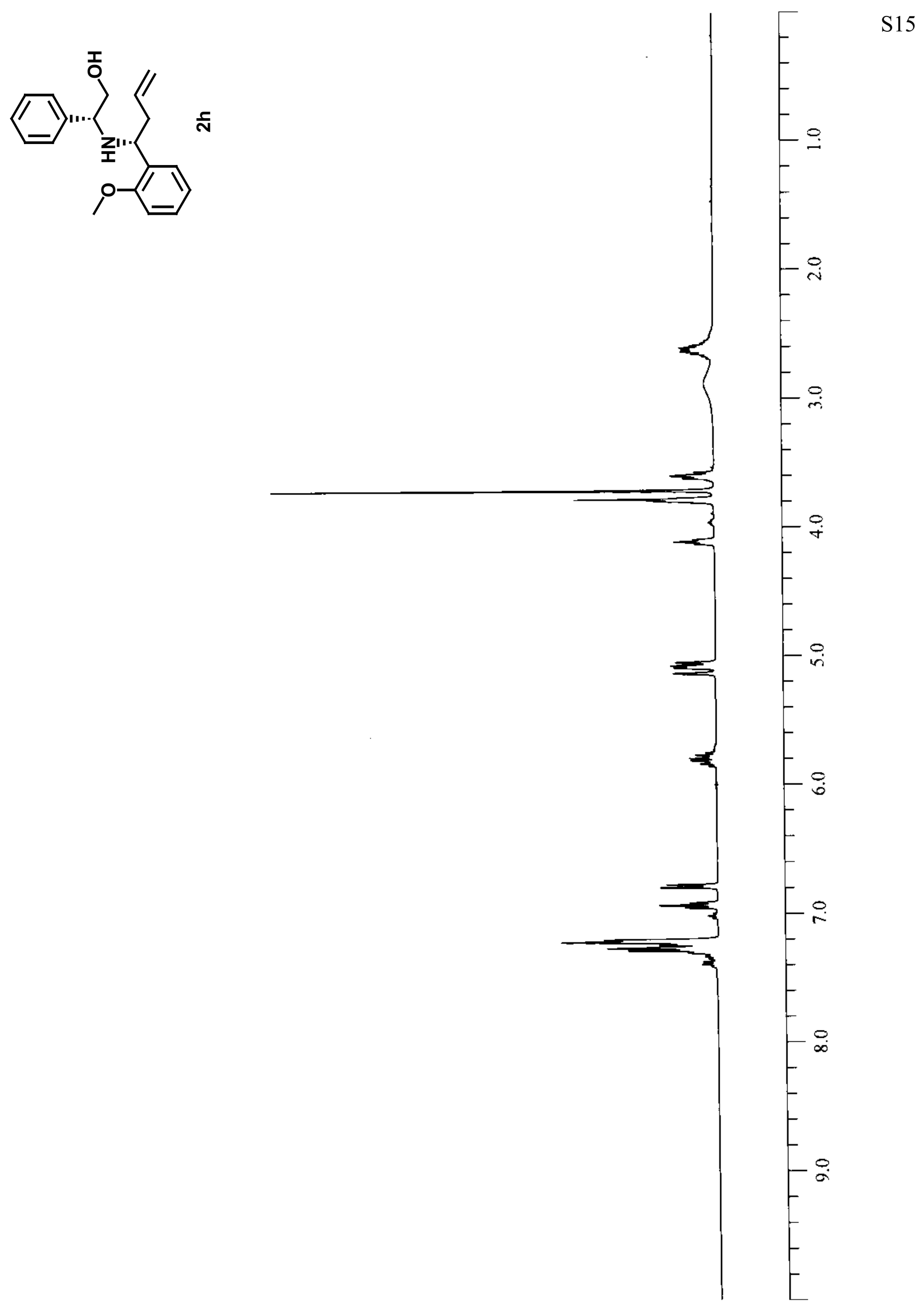

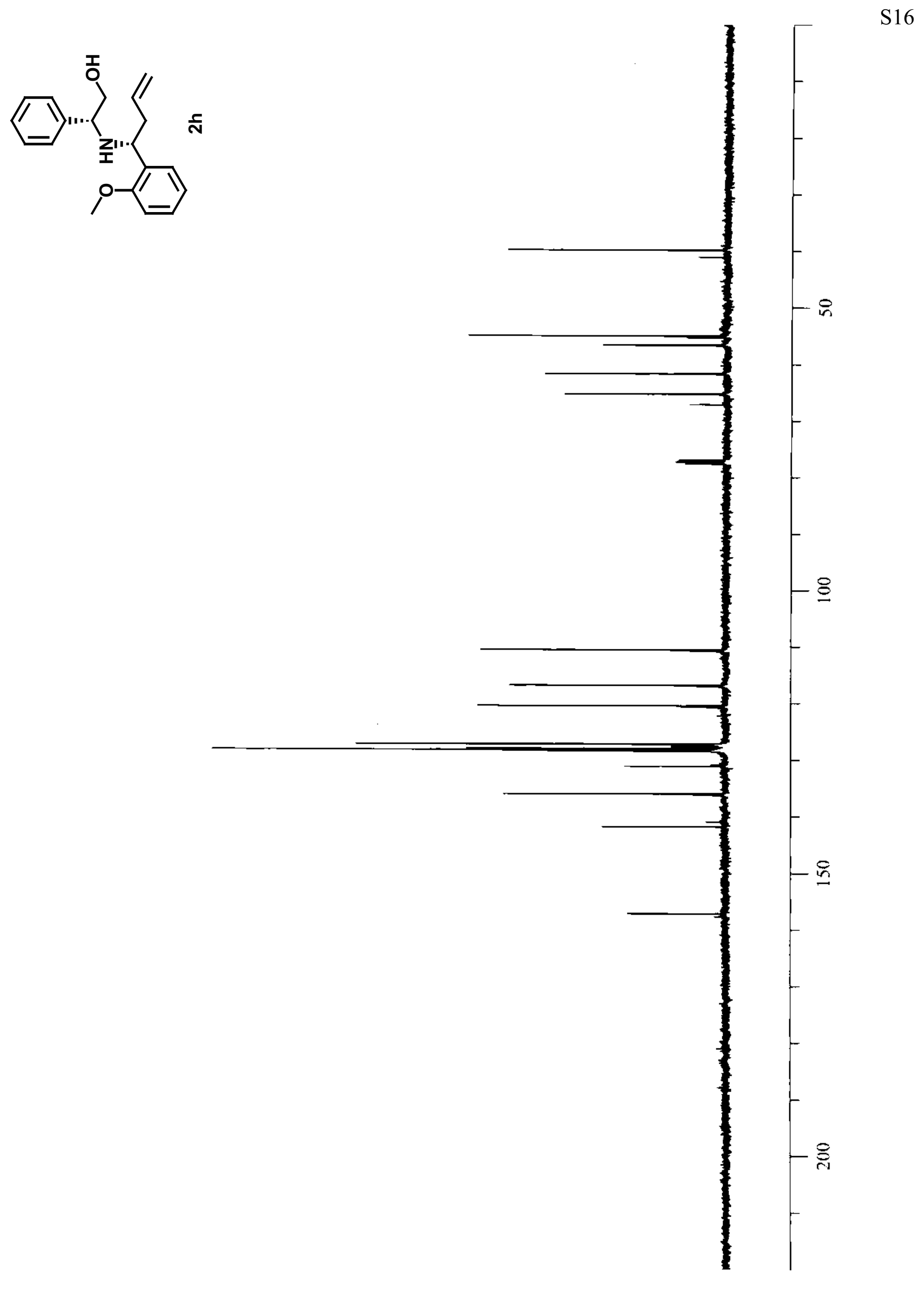

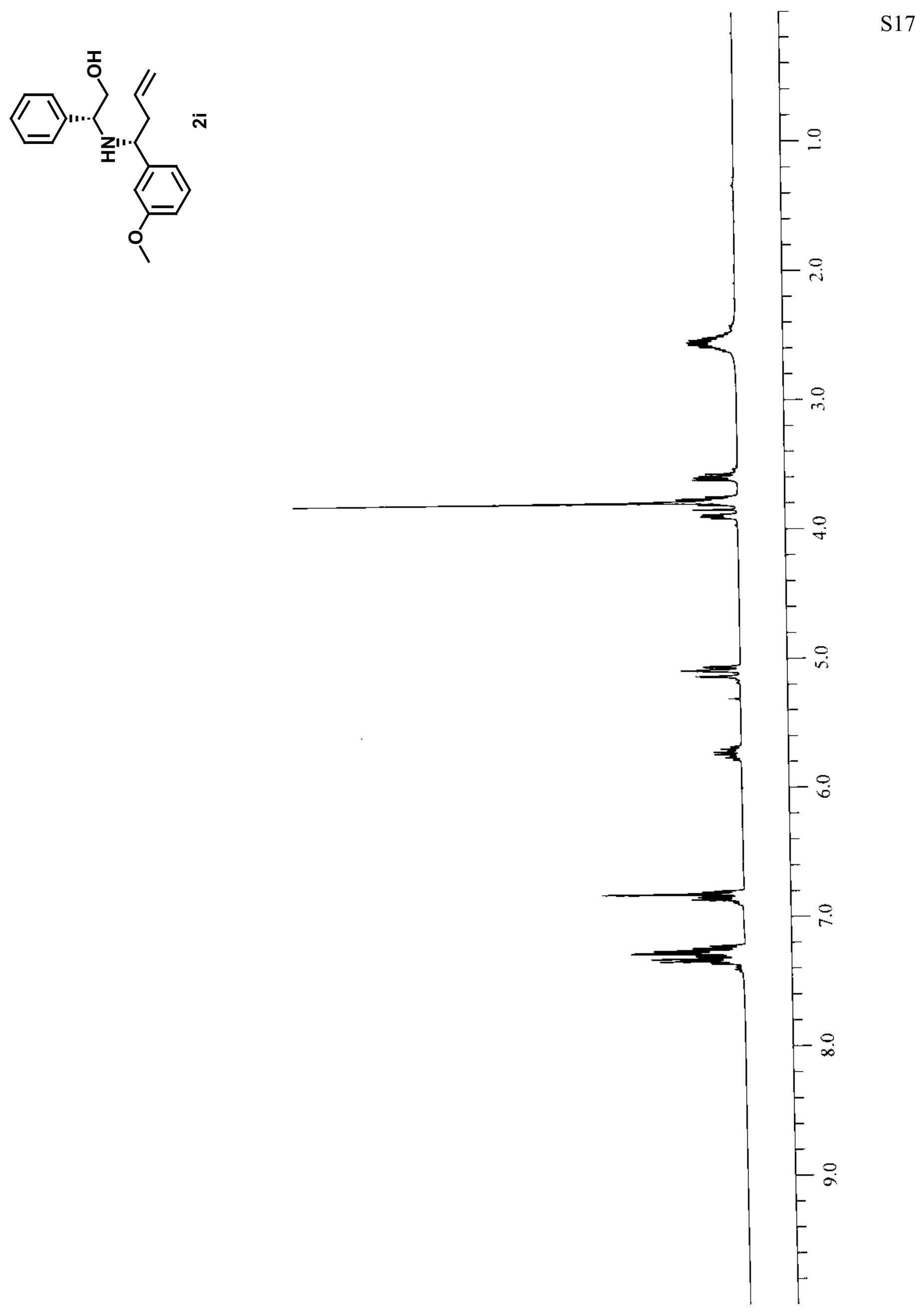

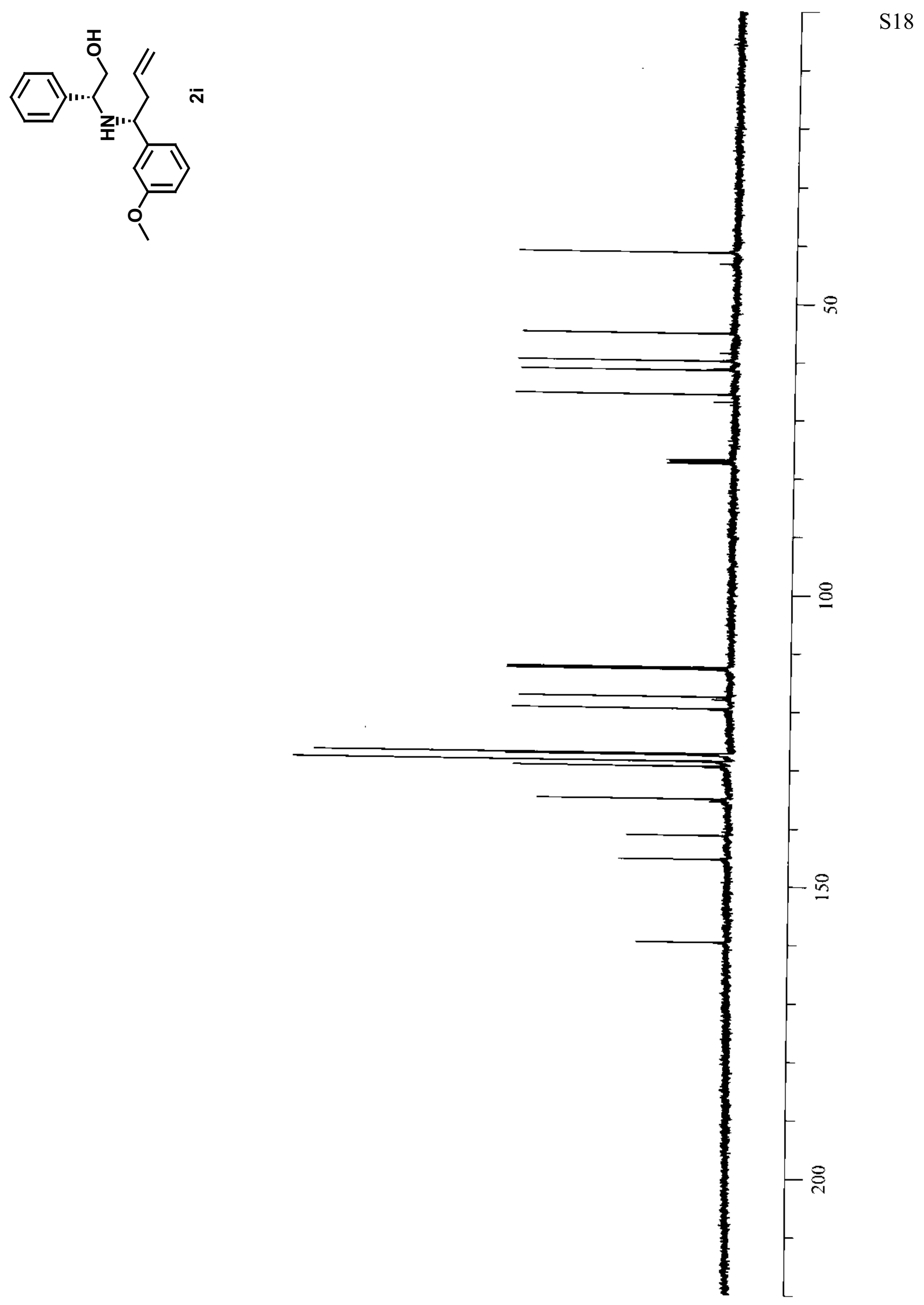

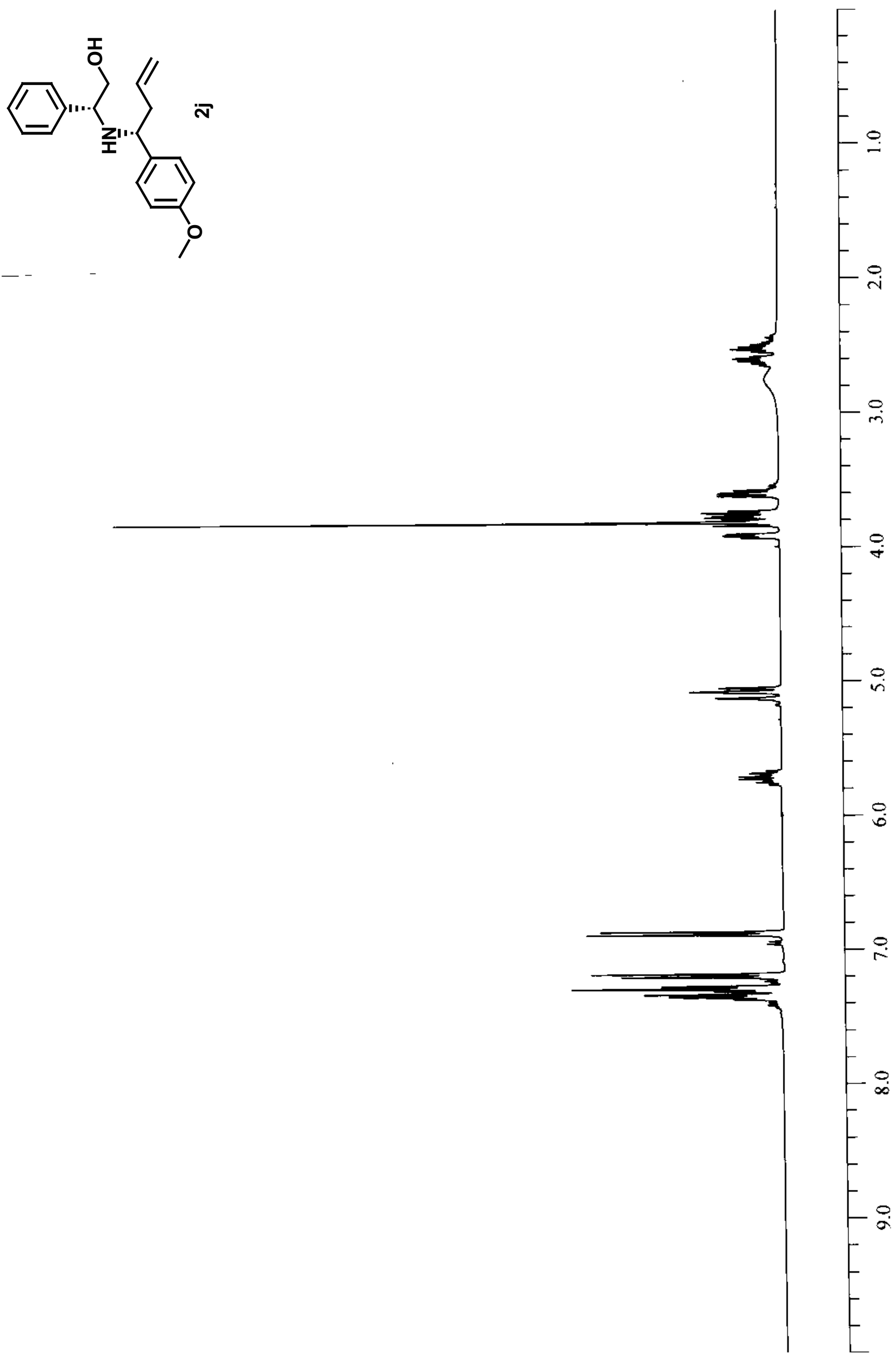

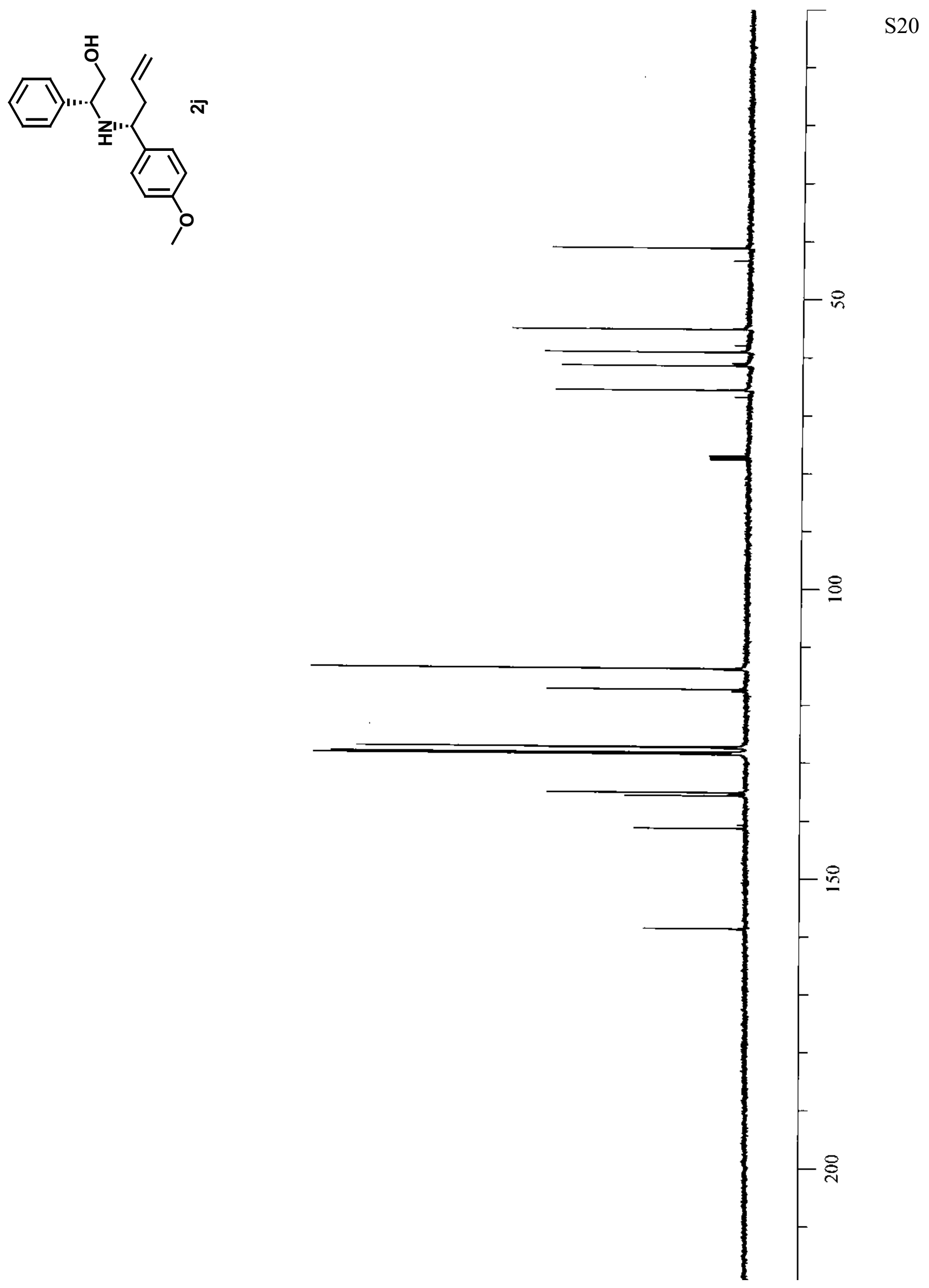

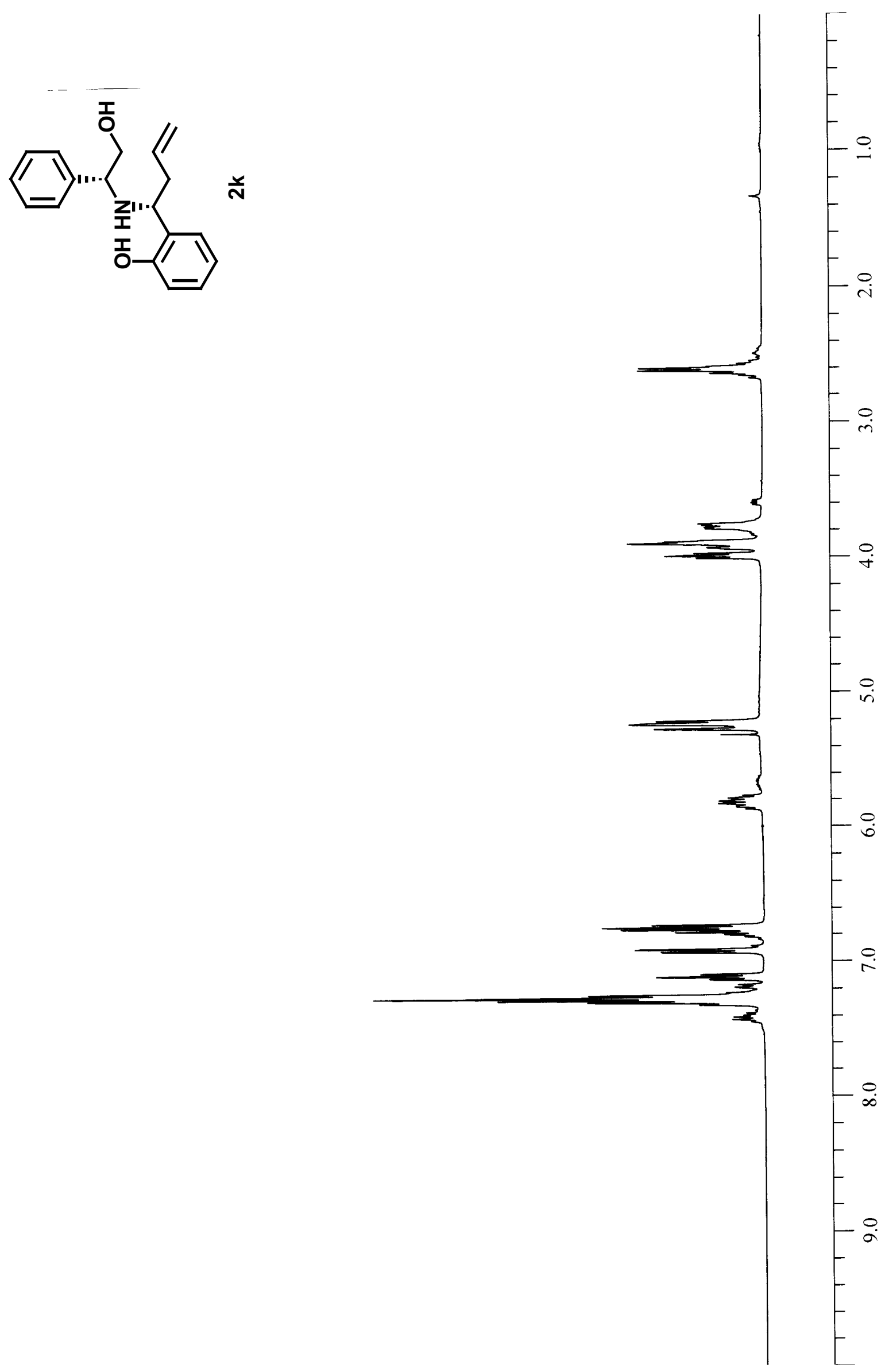

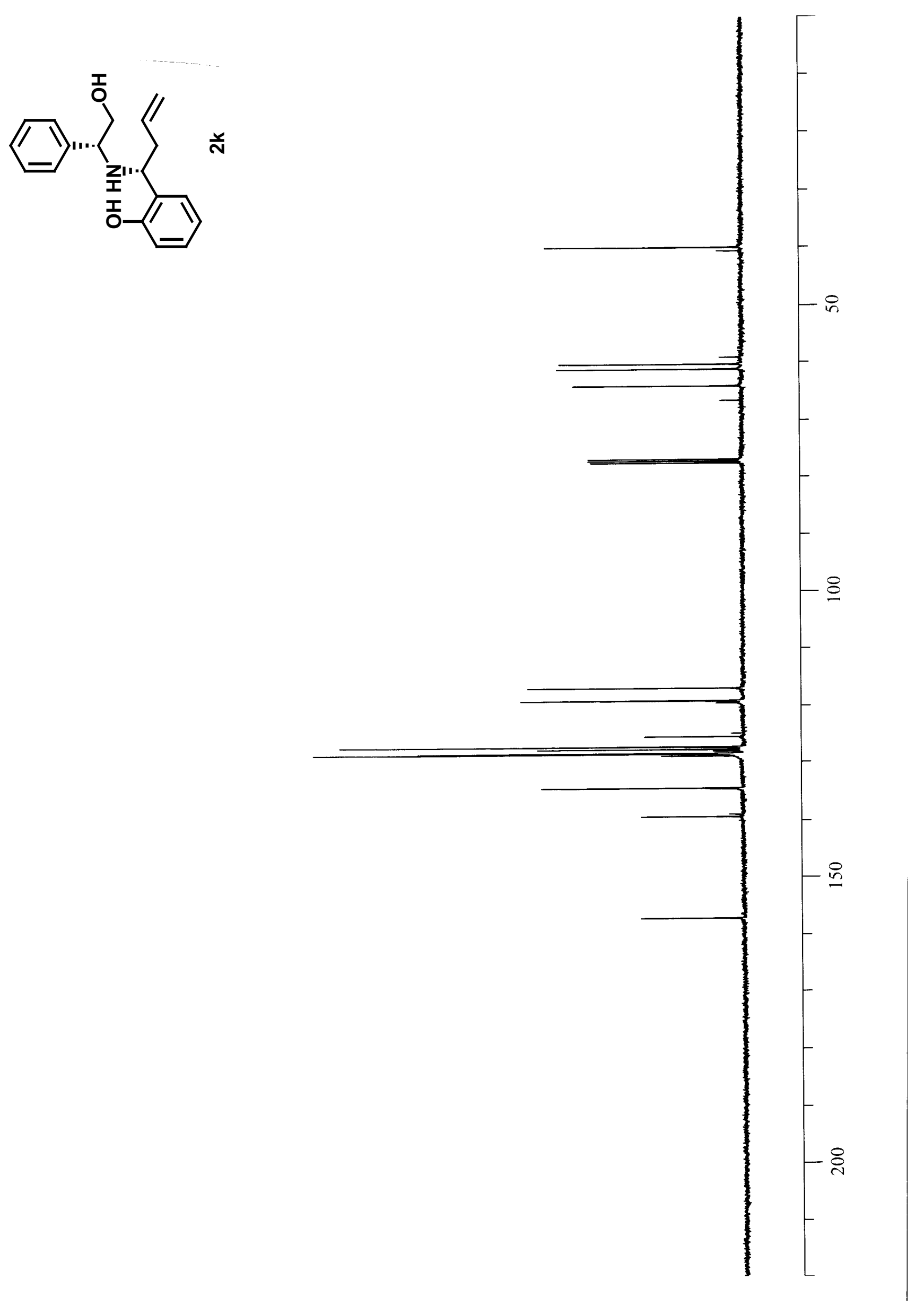

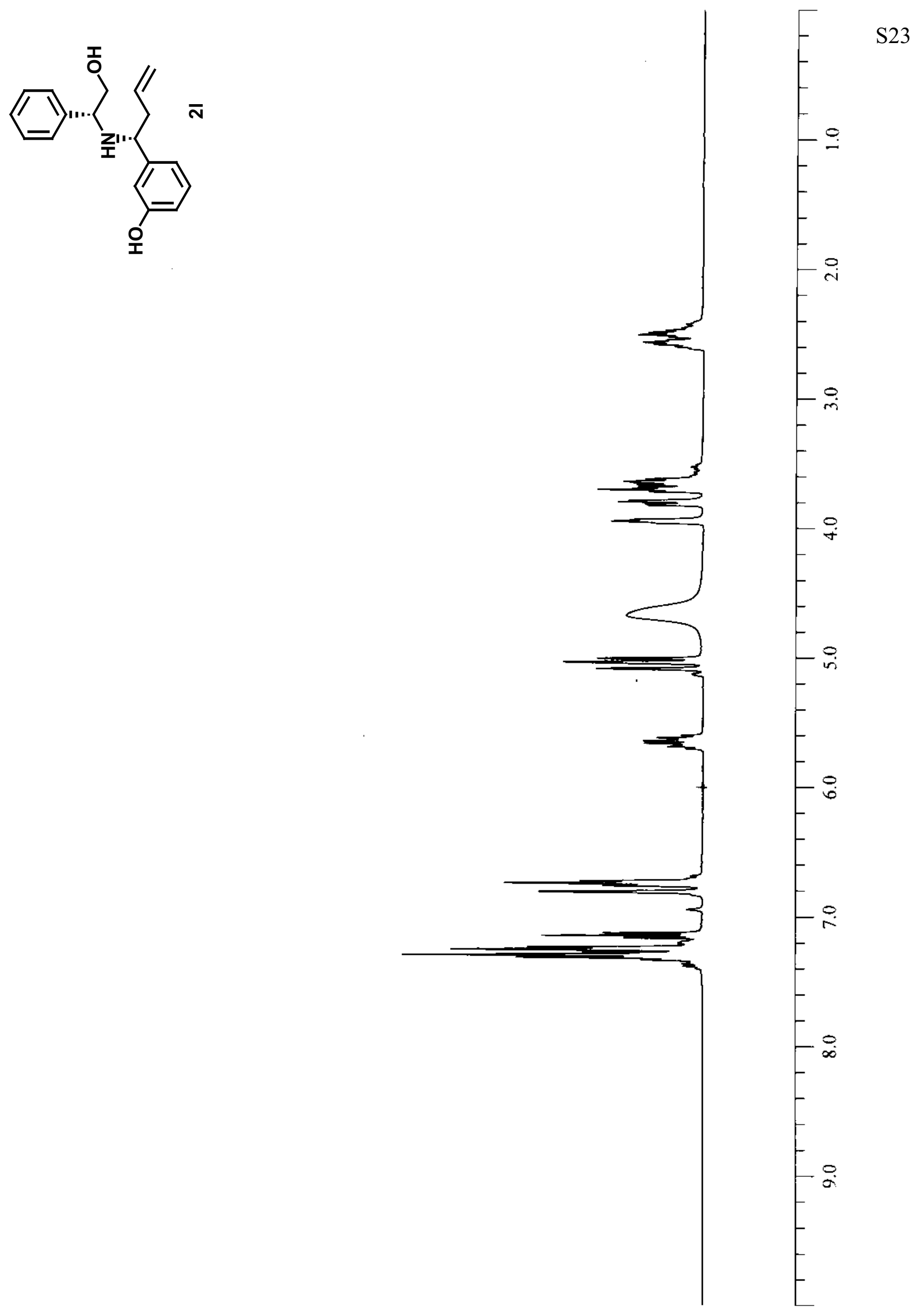

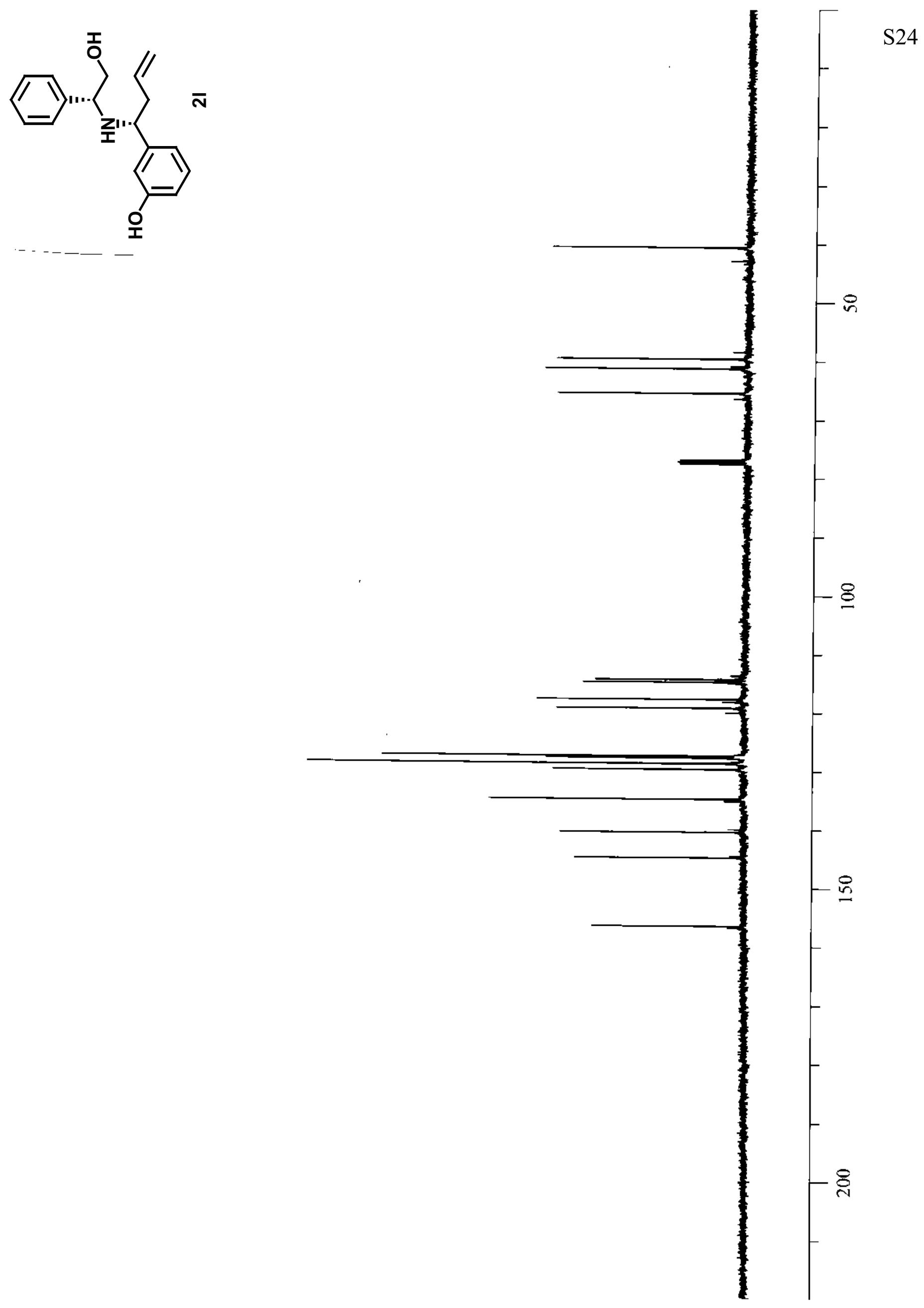

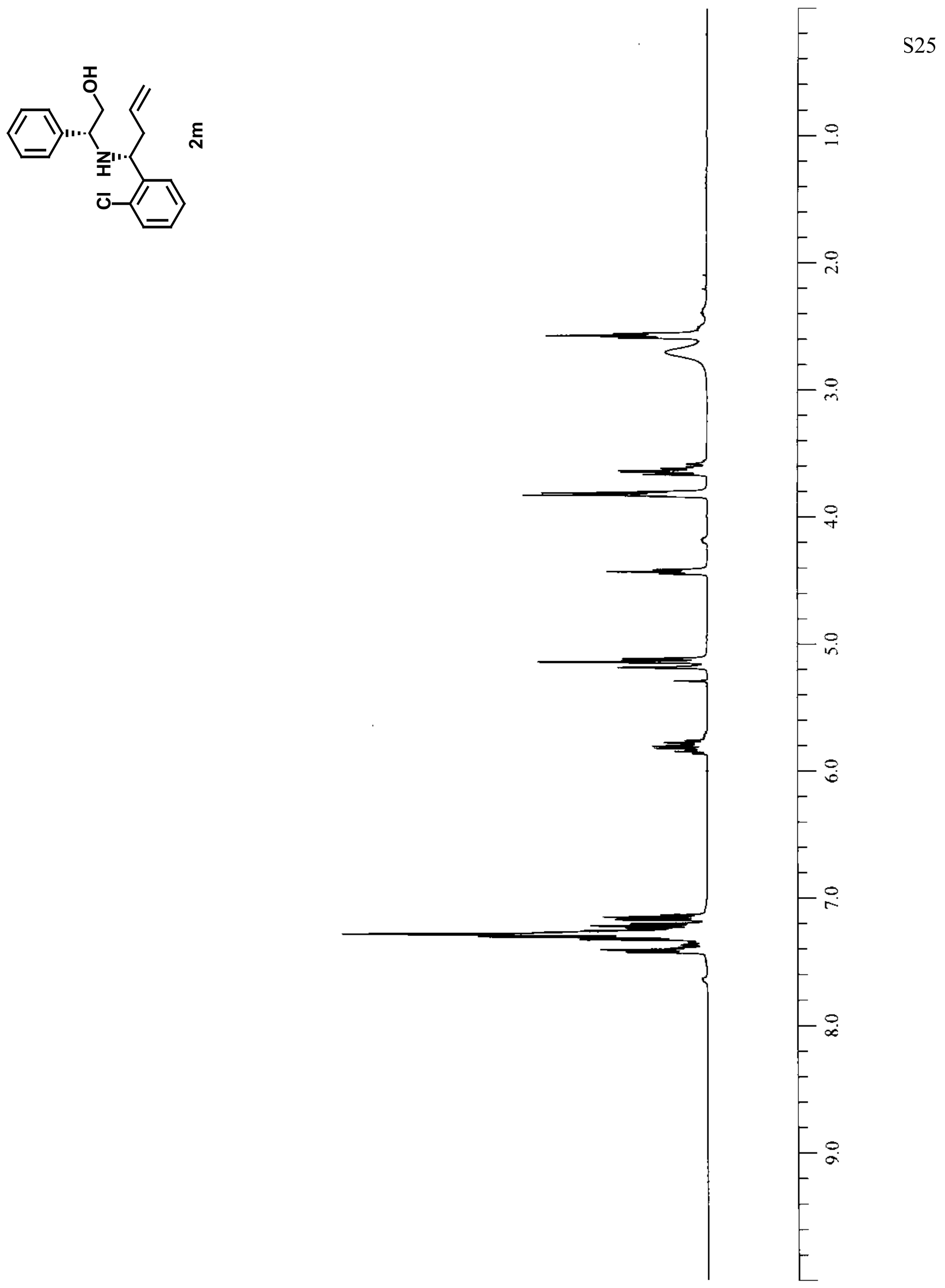

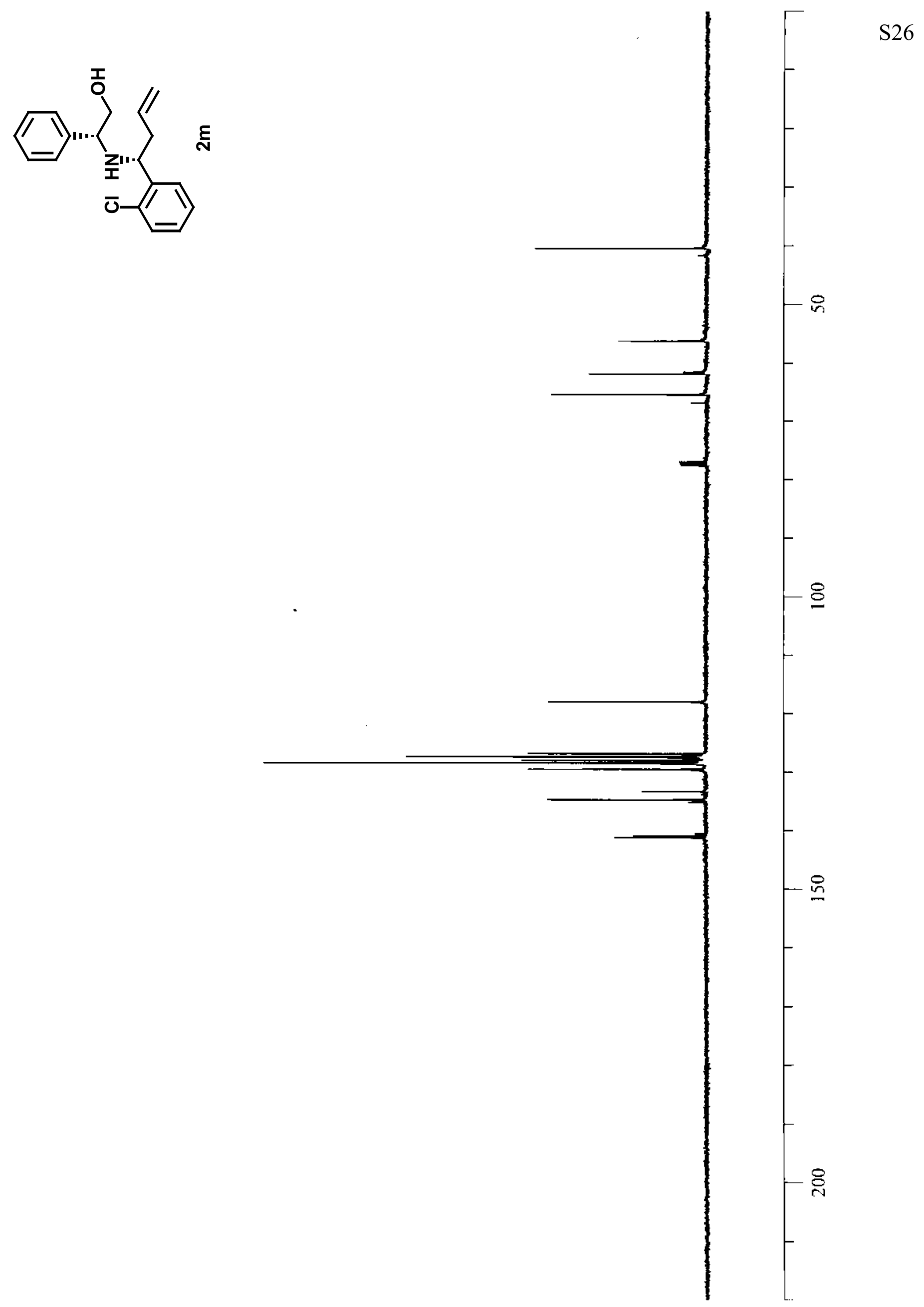

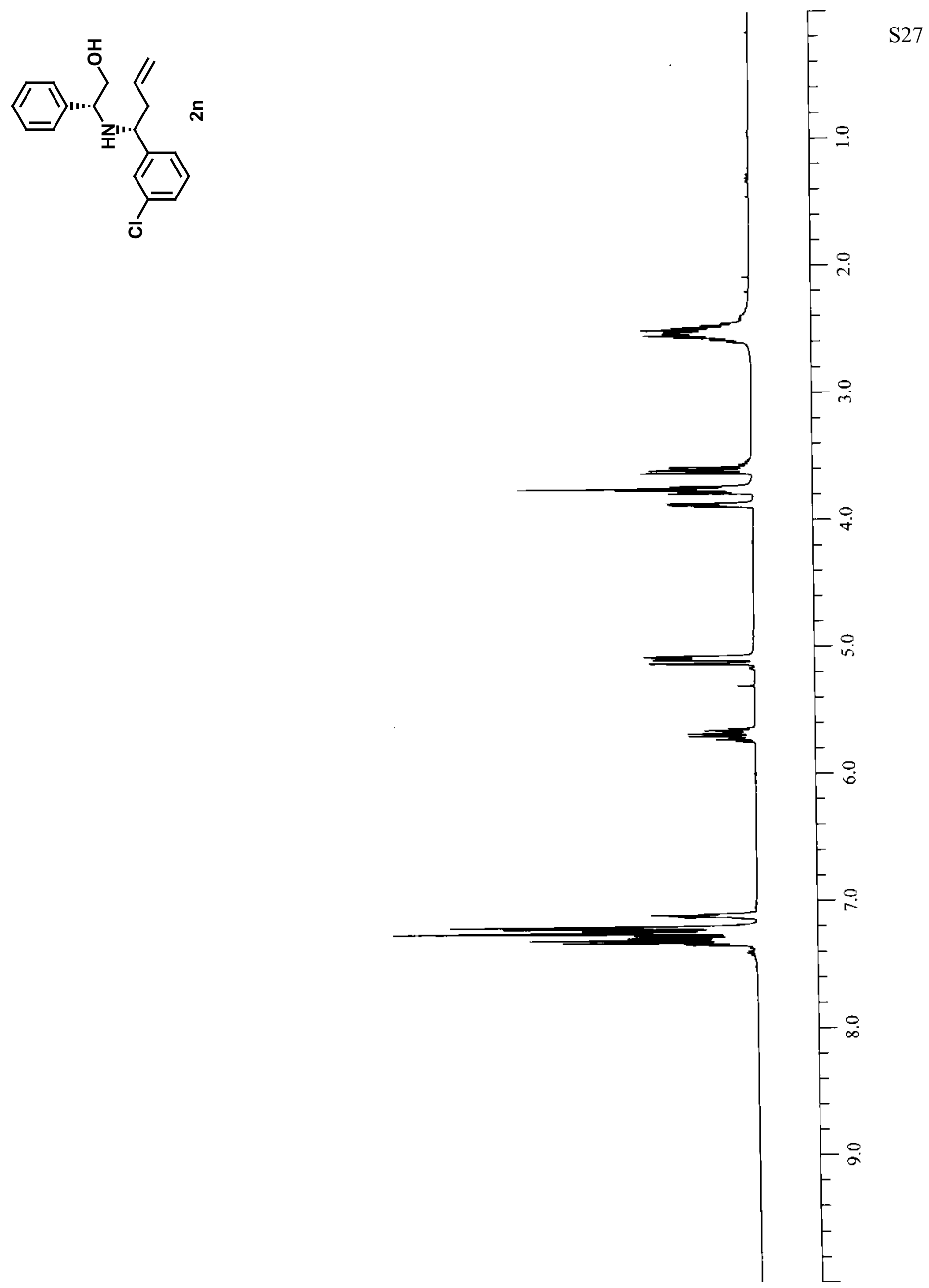

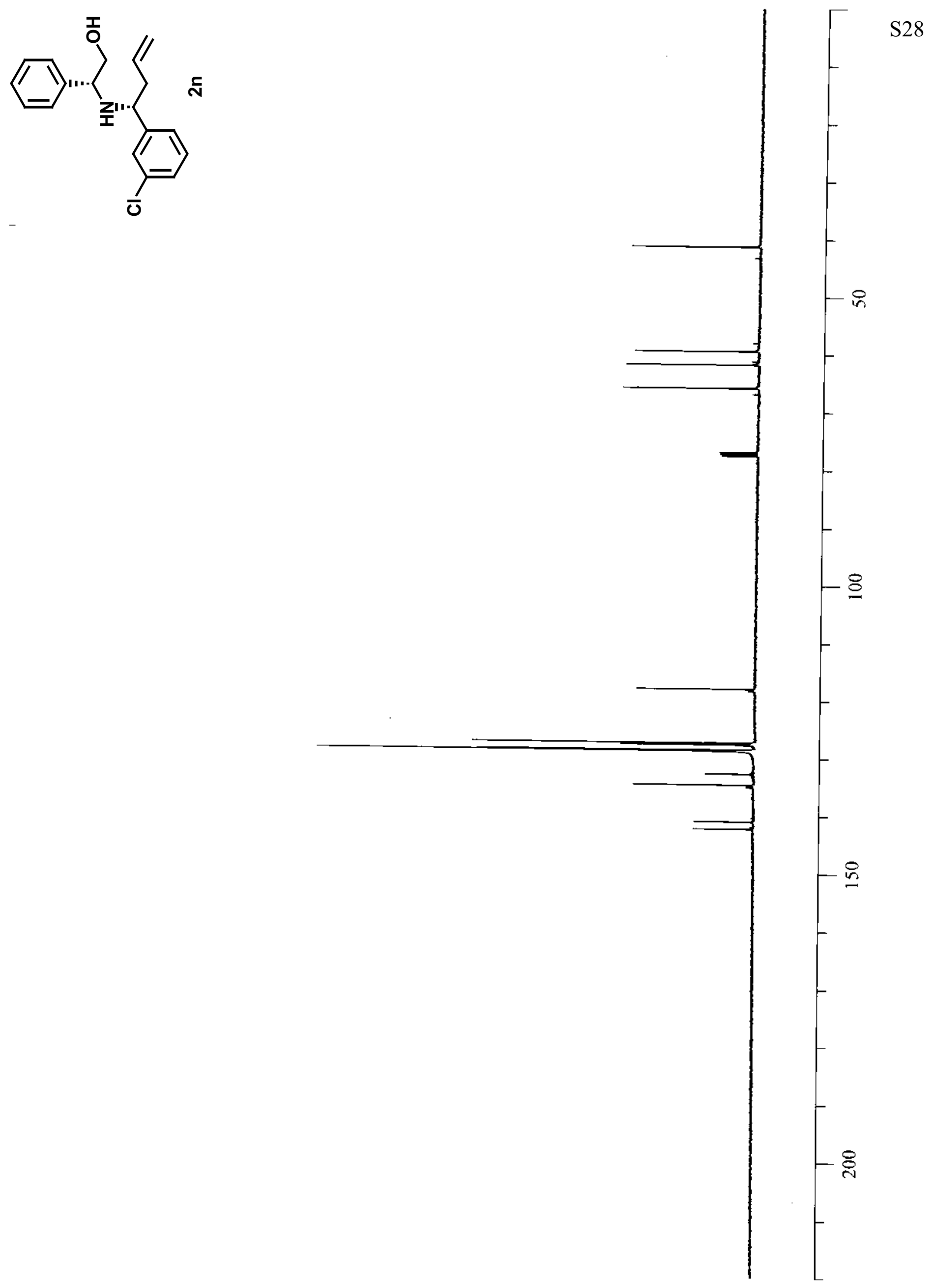

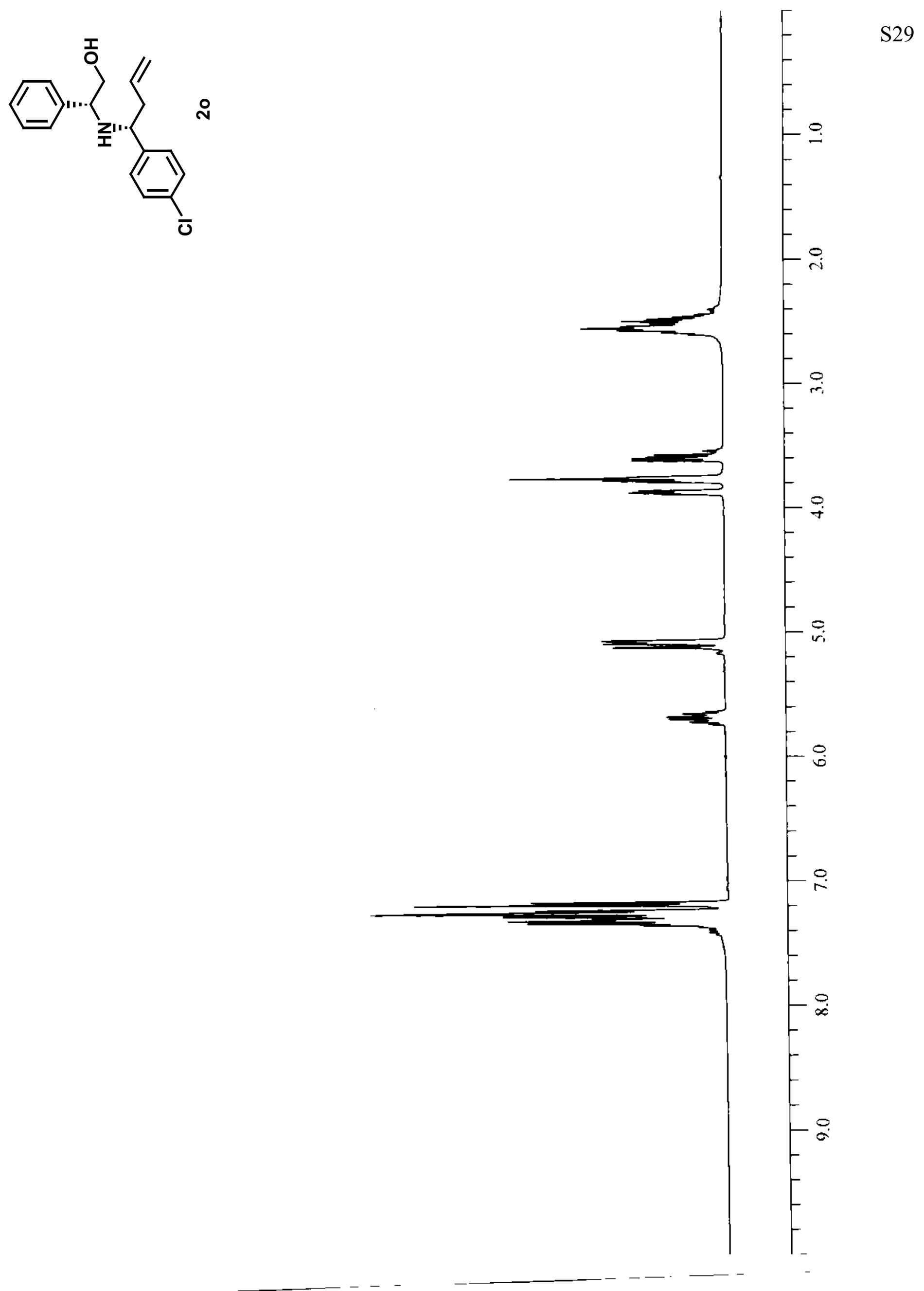

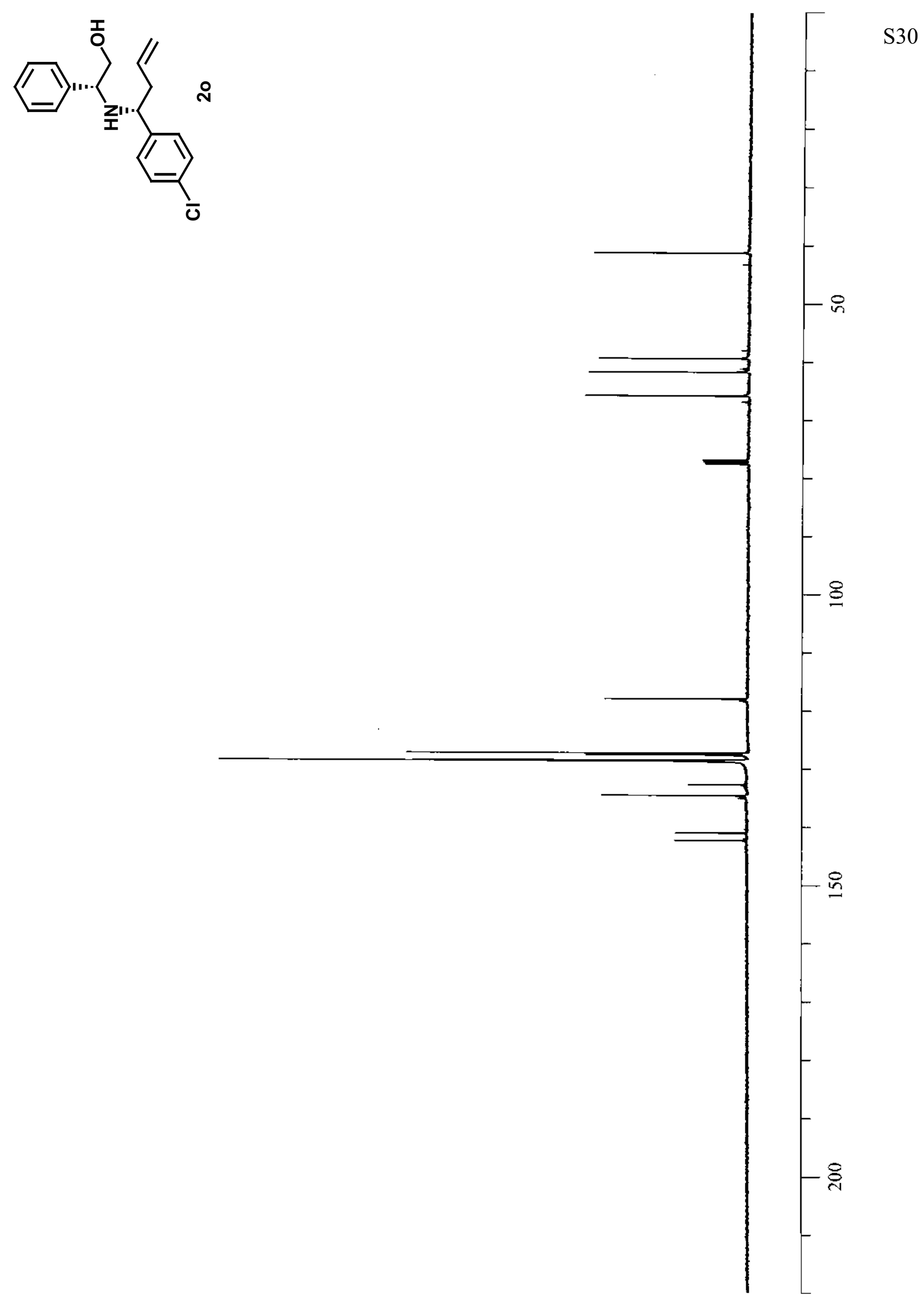

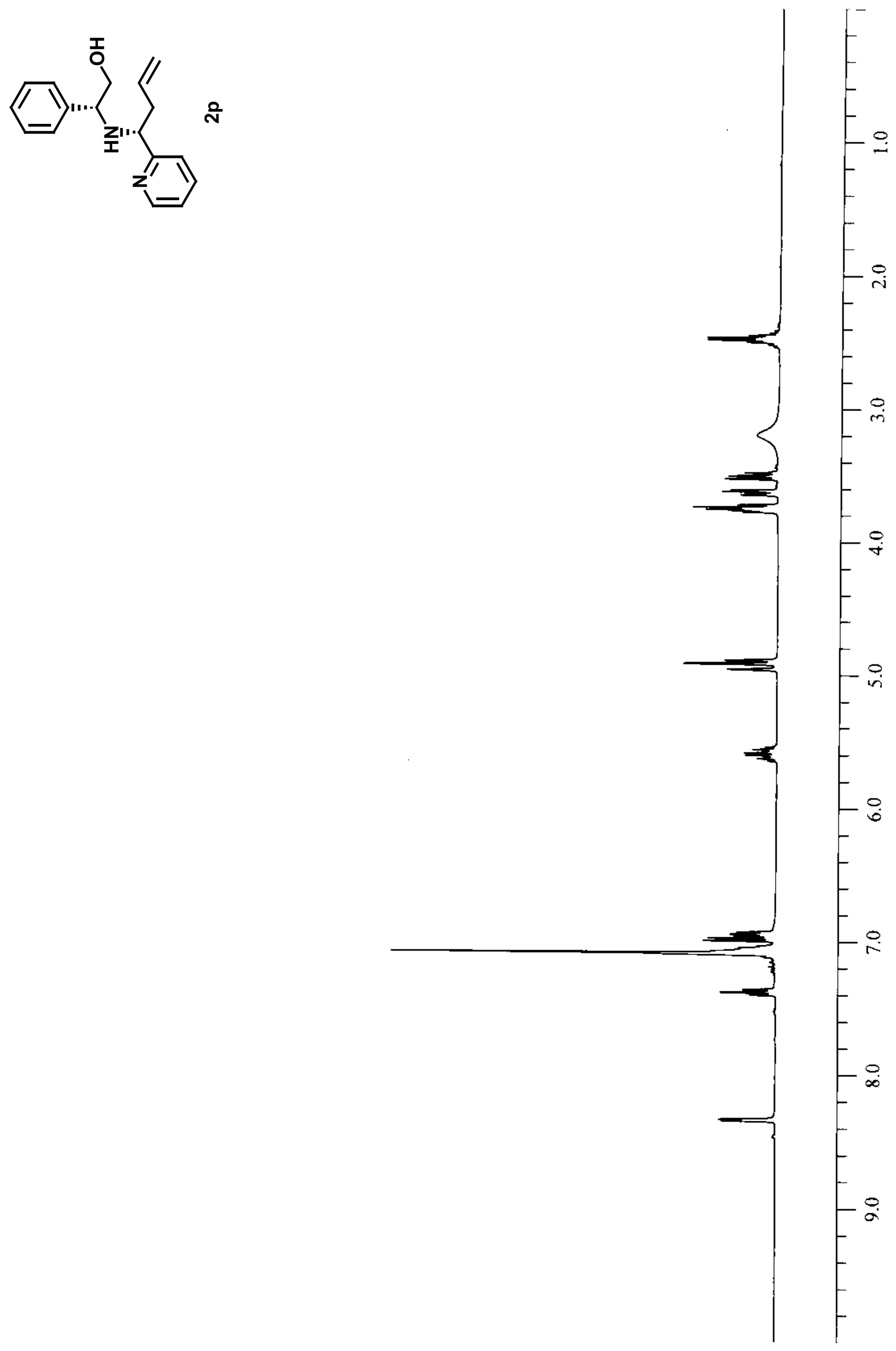

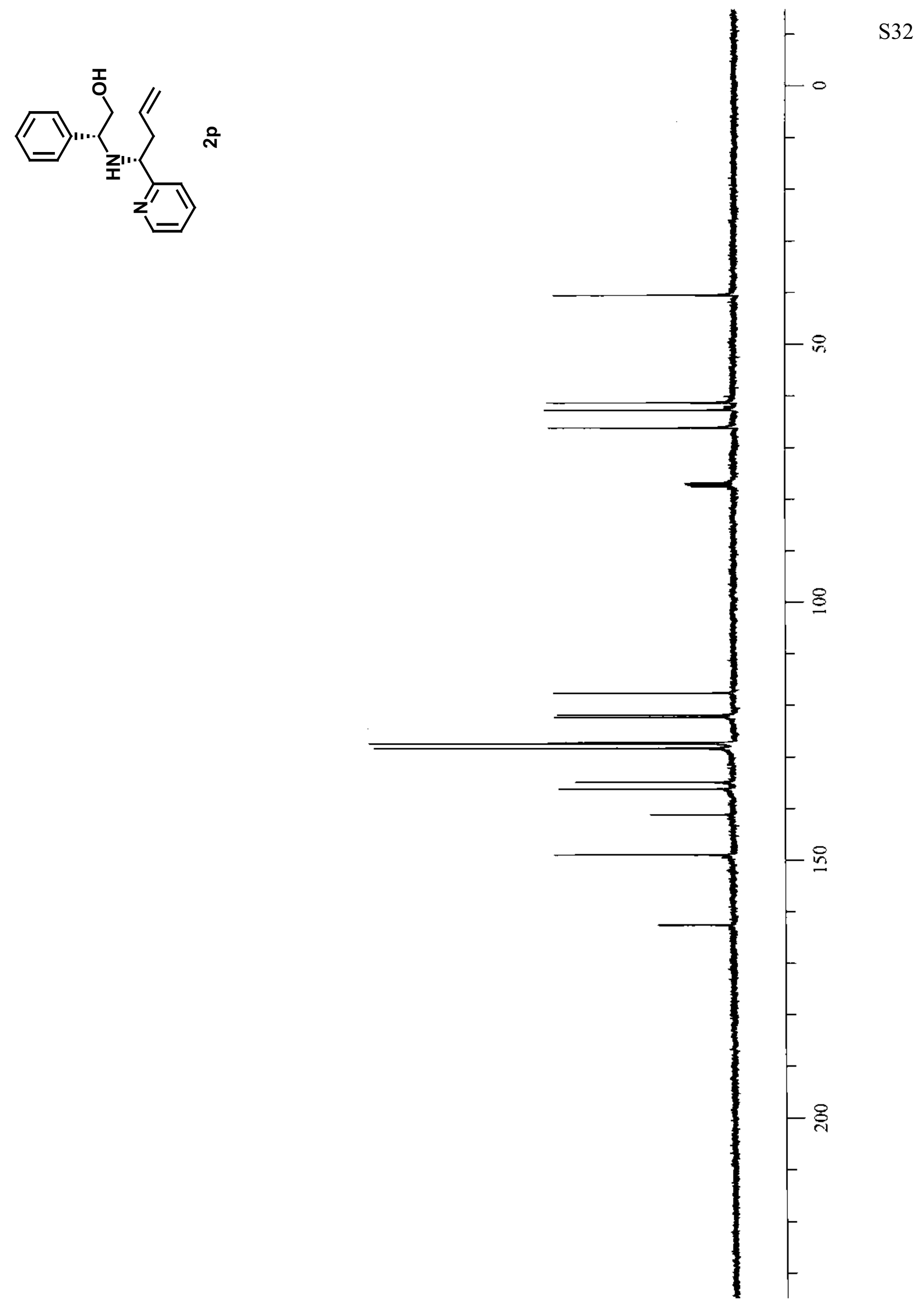

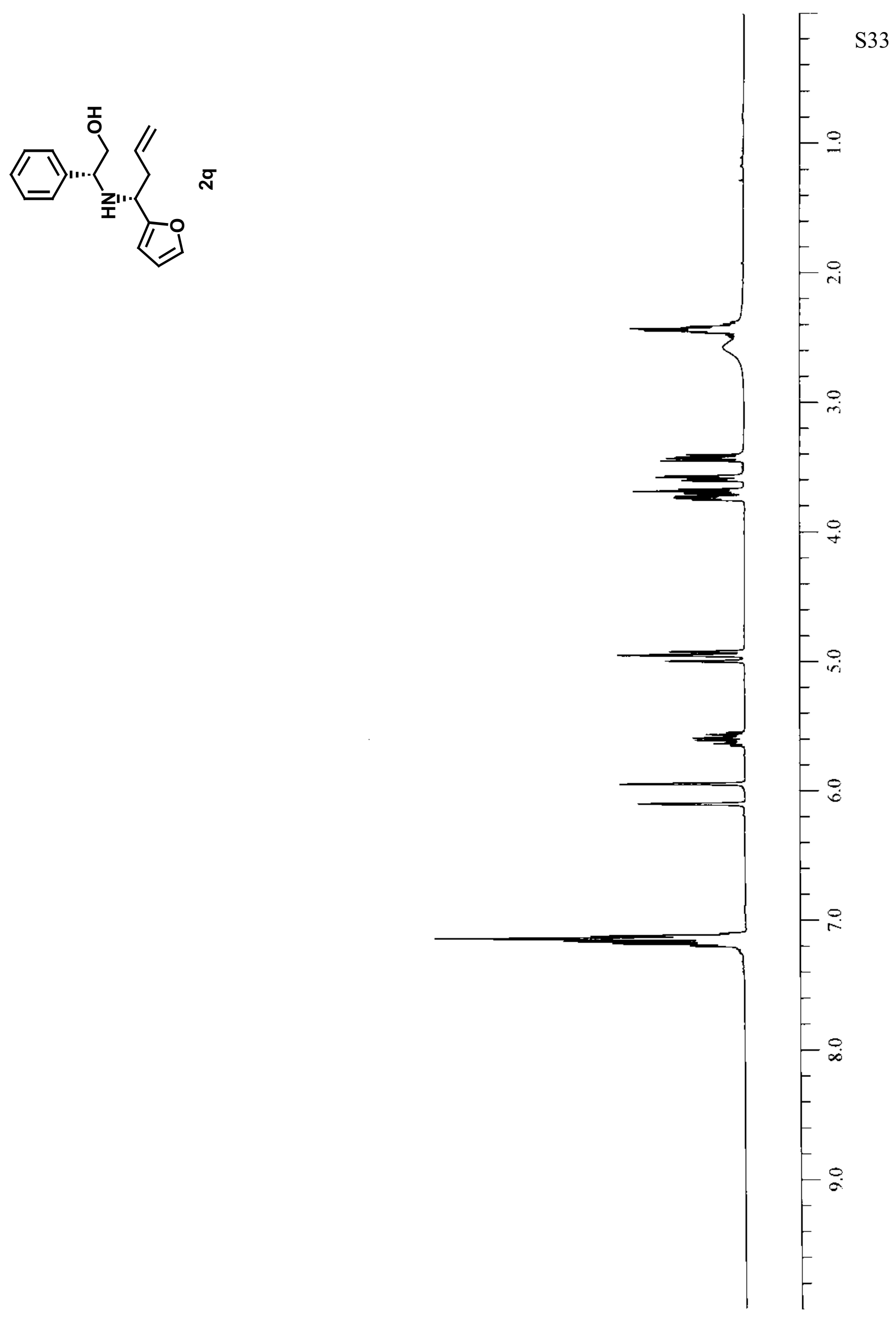


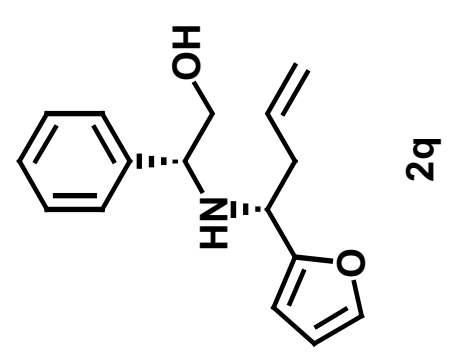

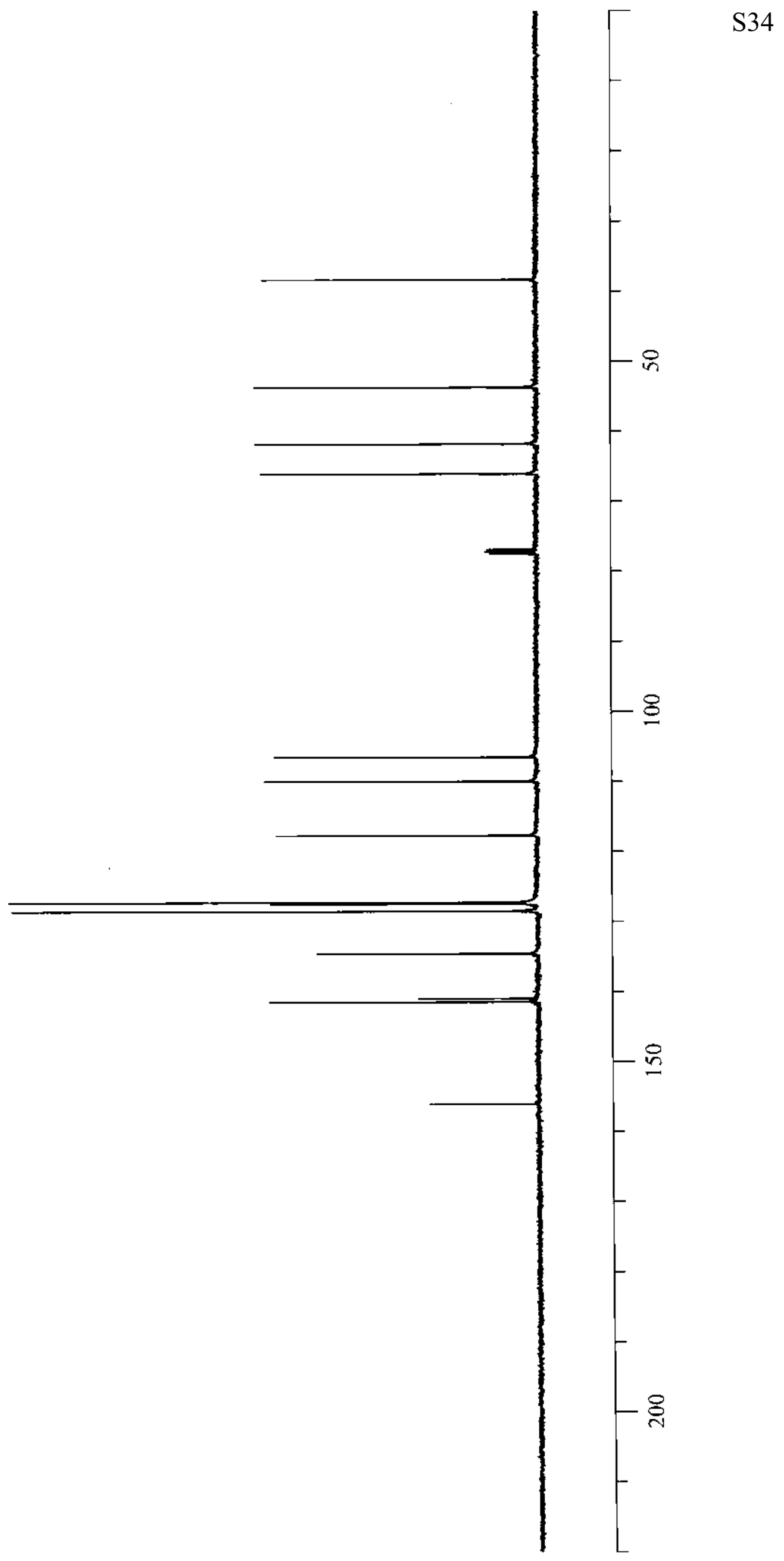



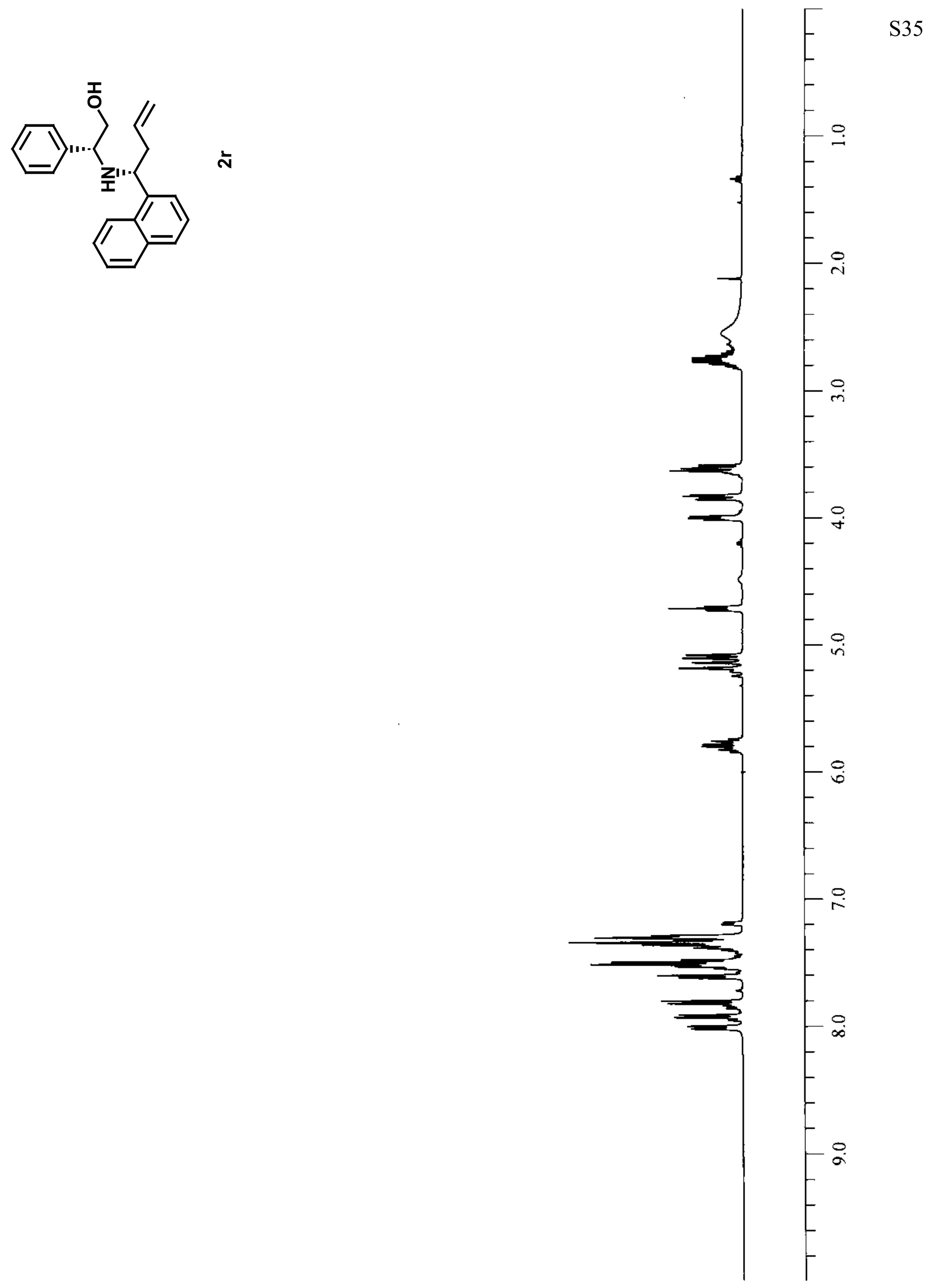


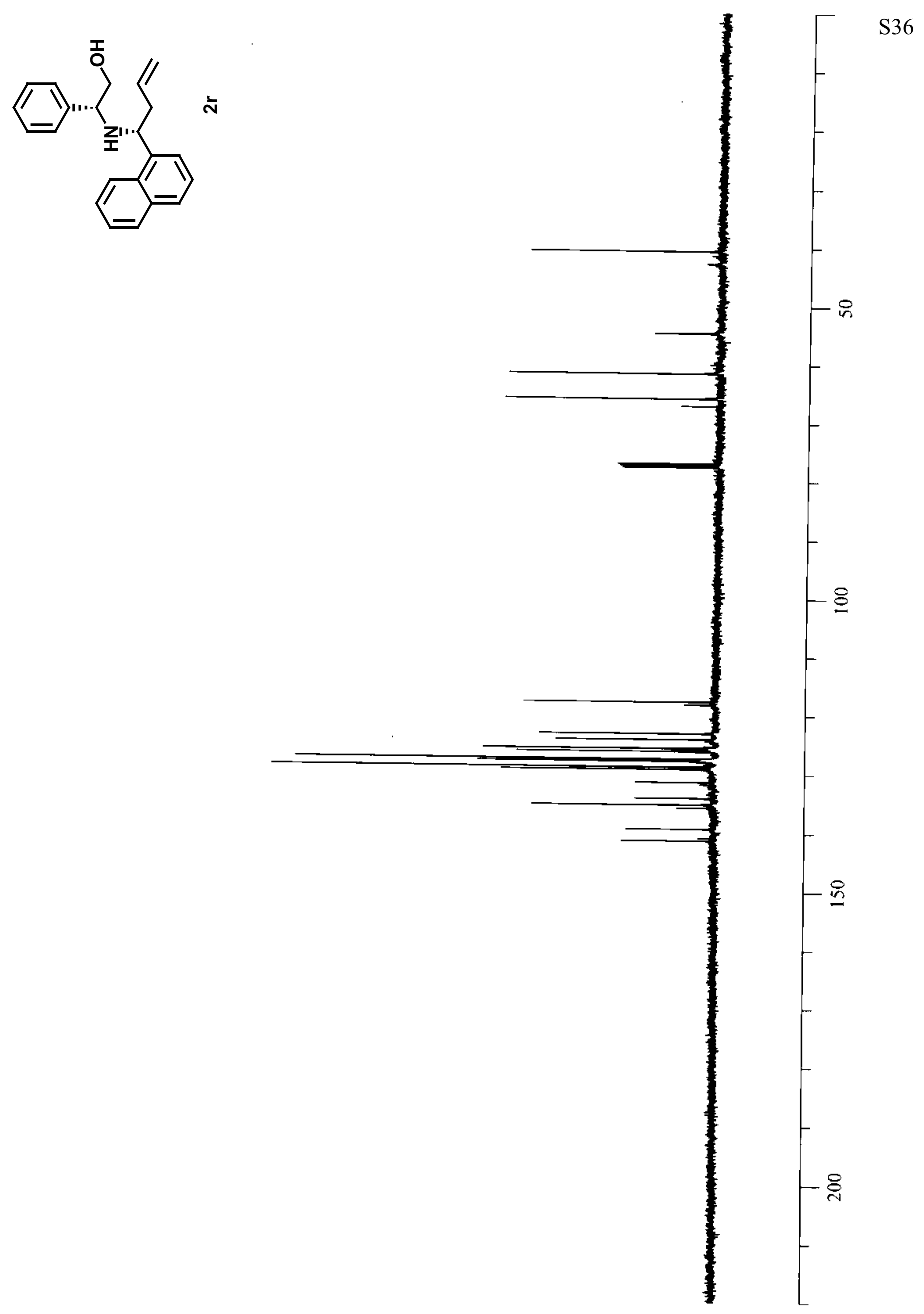



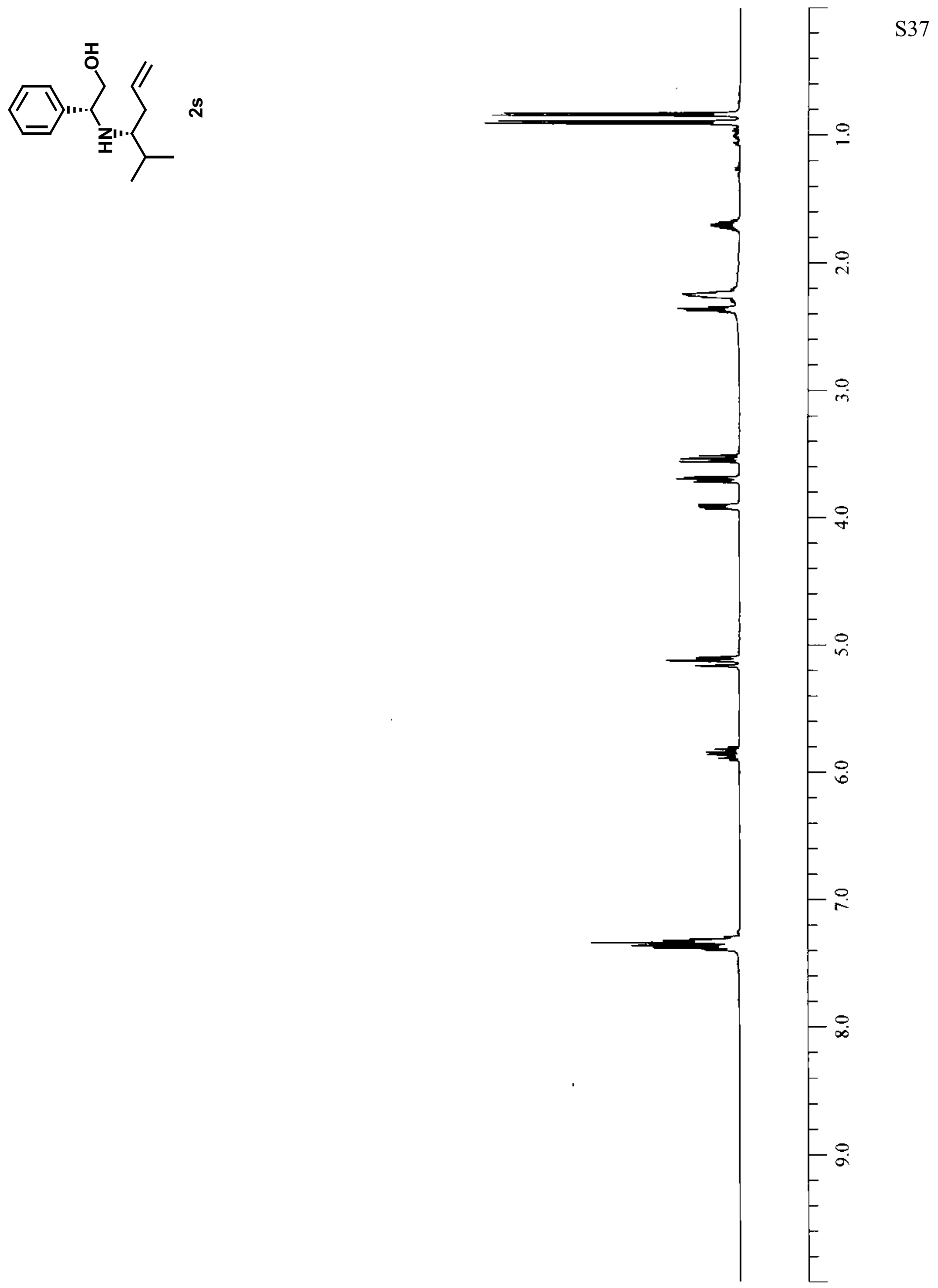

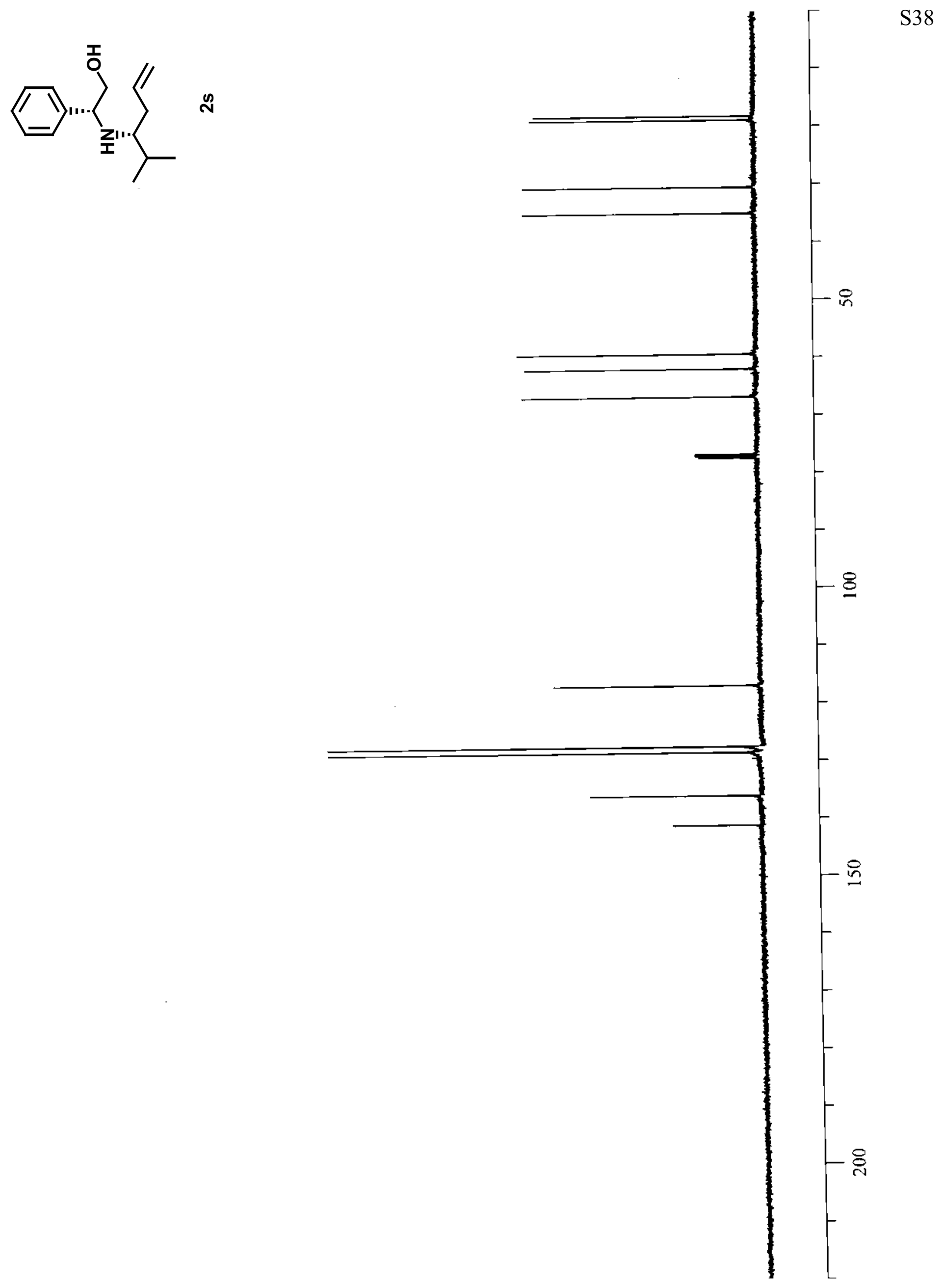

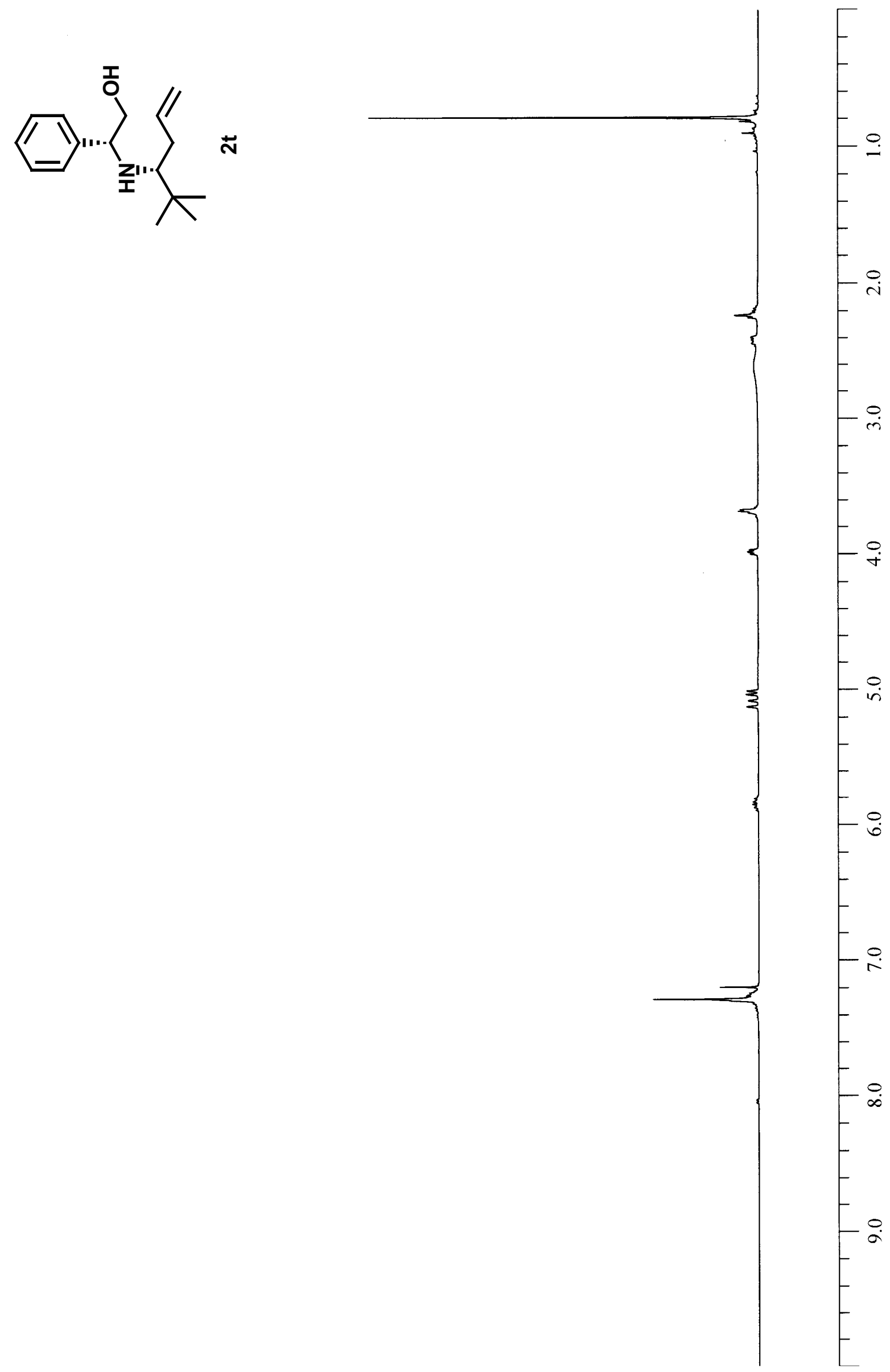


$$
{ }^{\prime \prime}=
$$



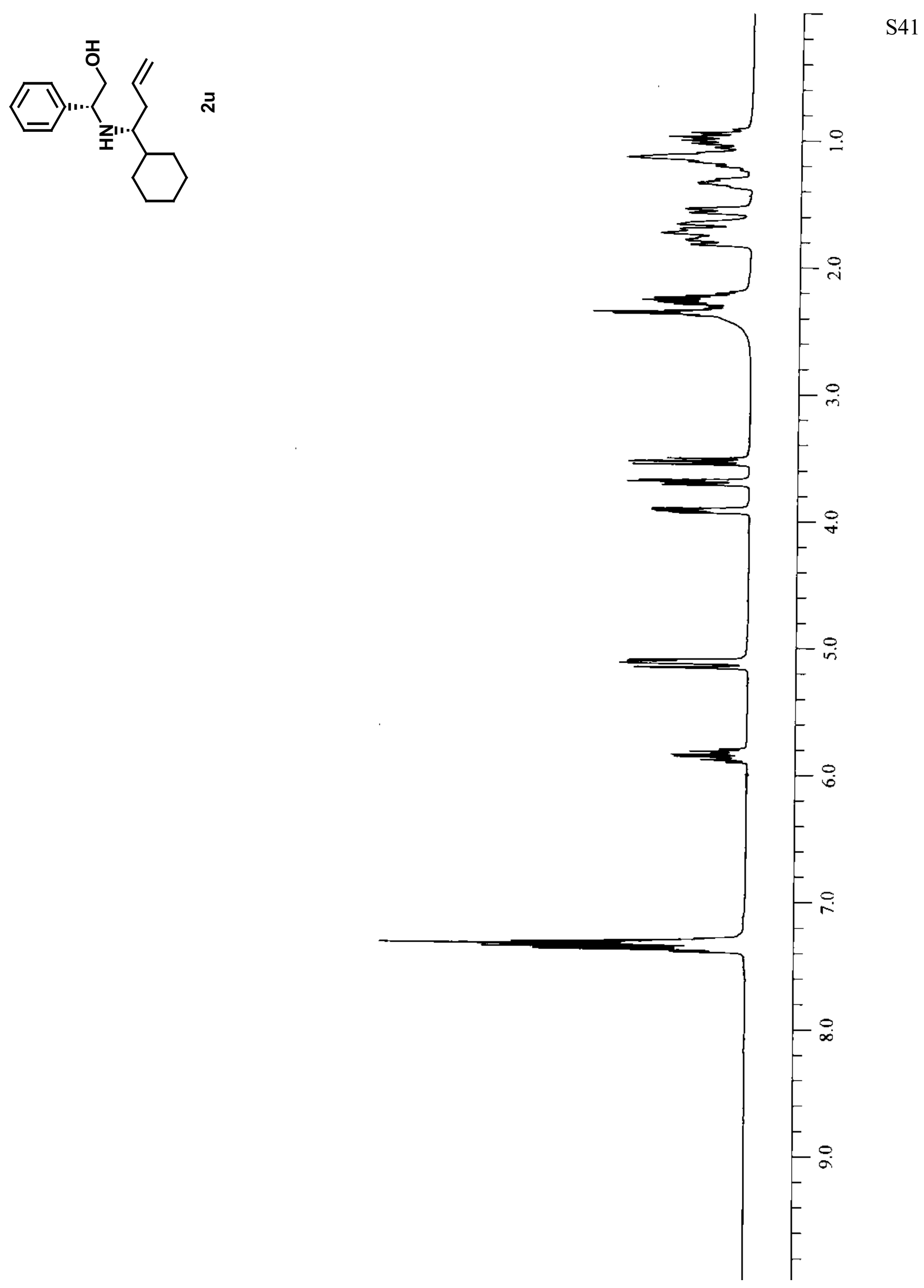


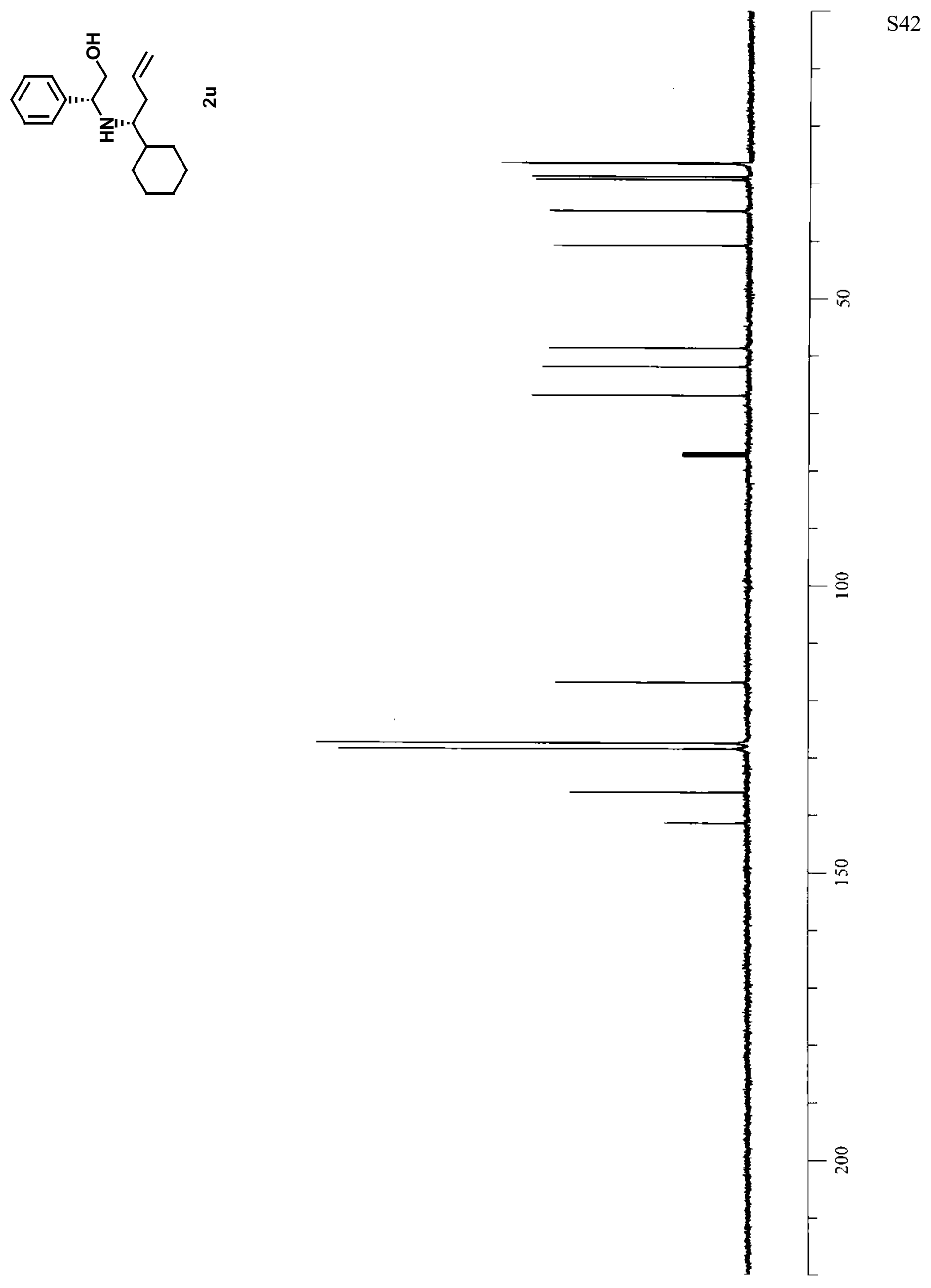



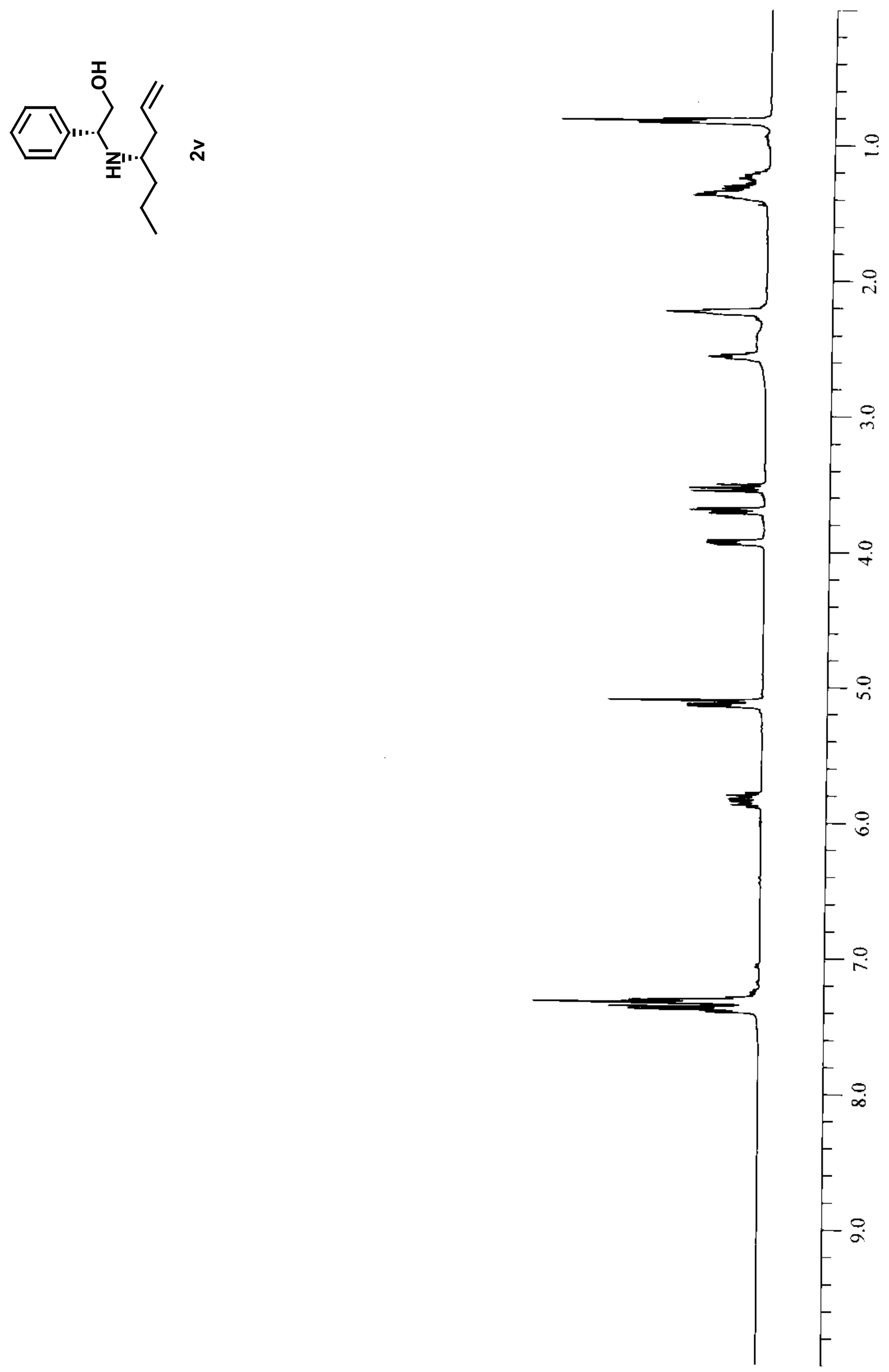

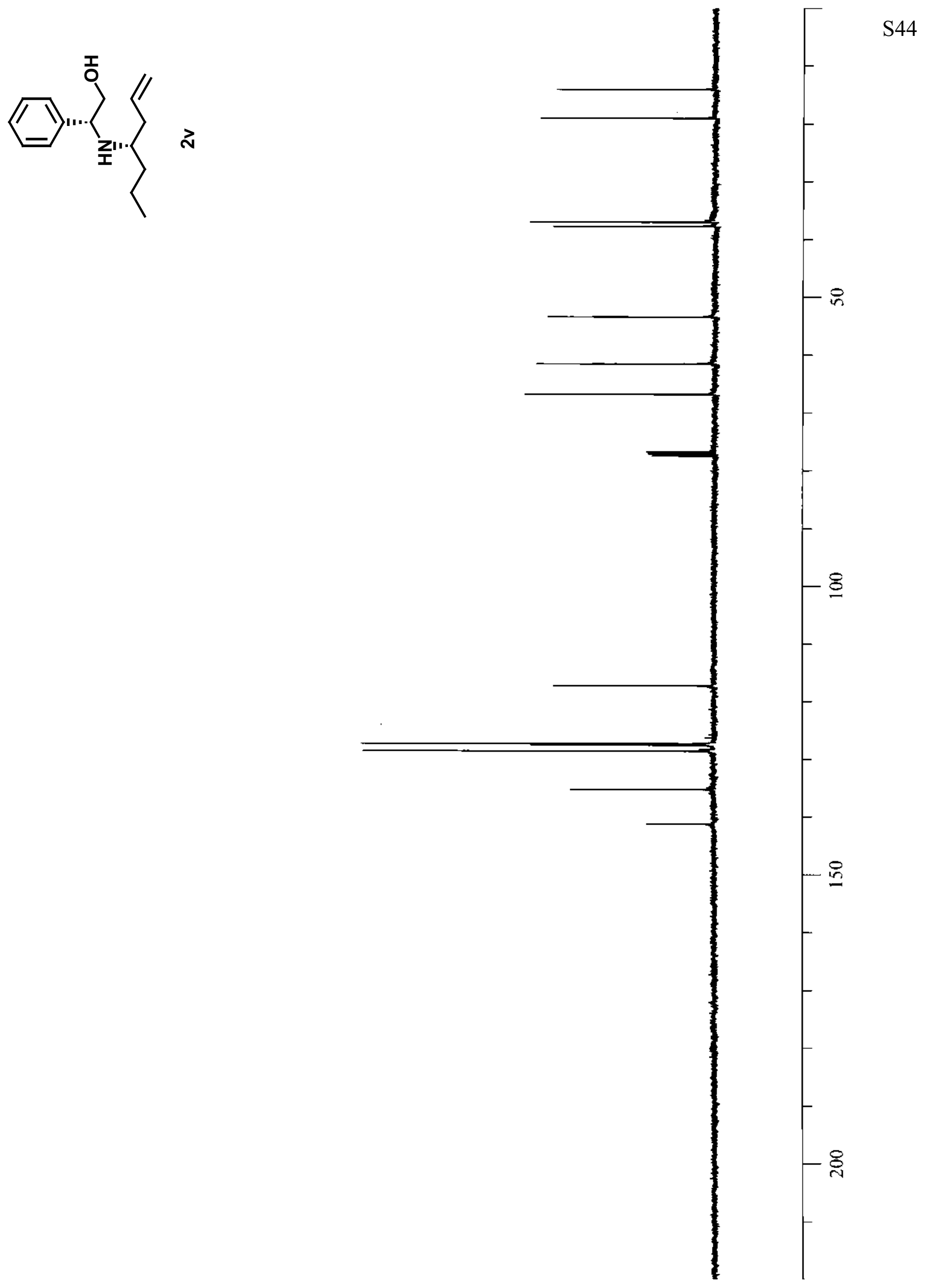

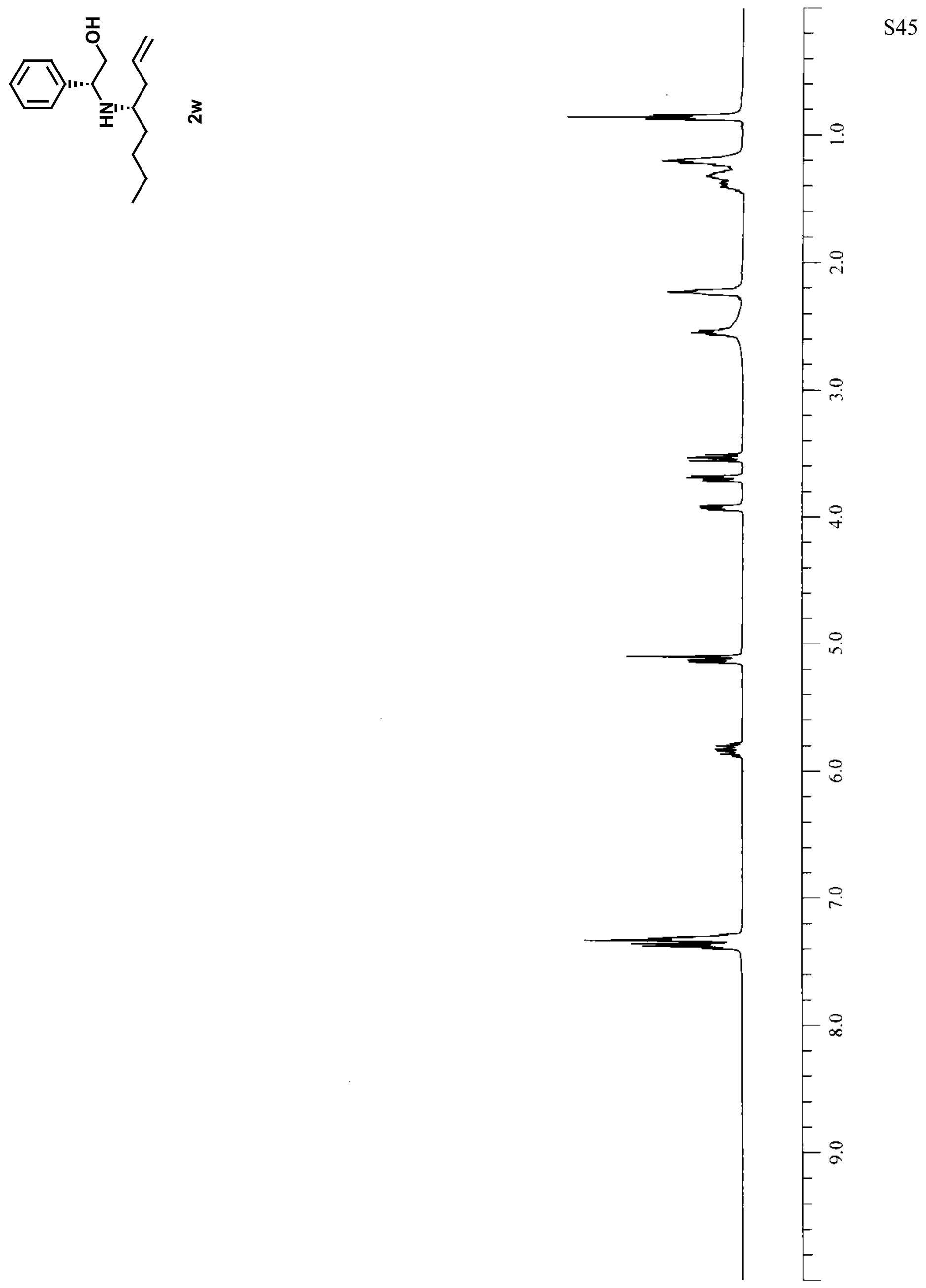

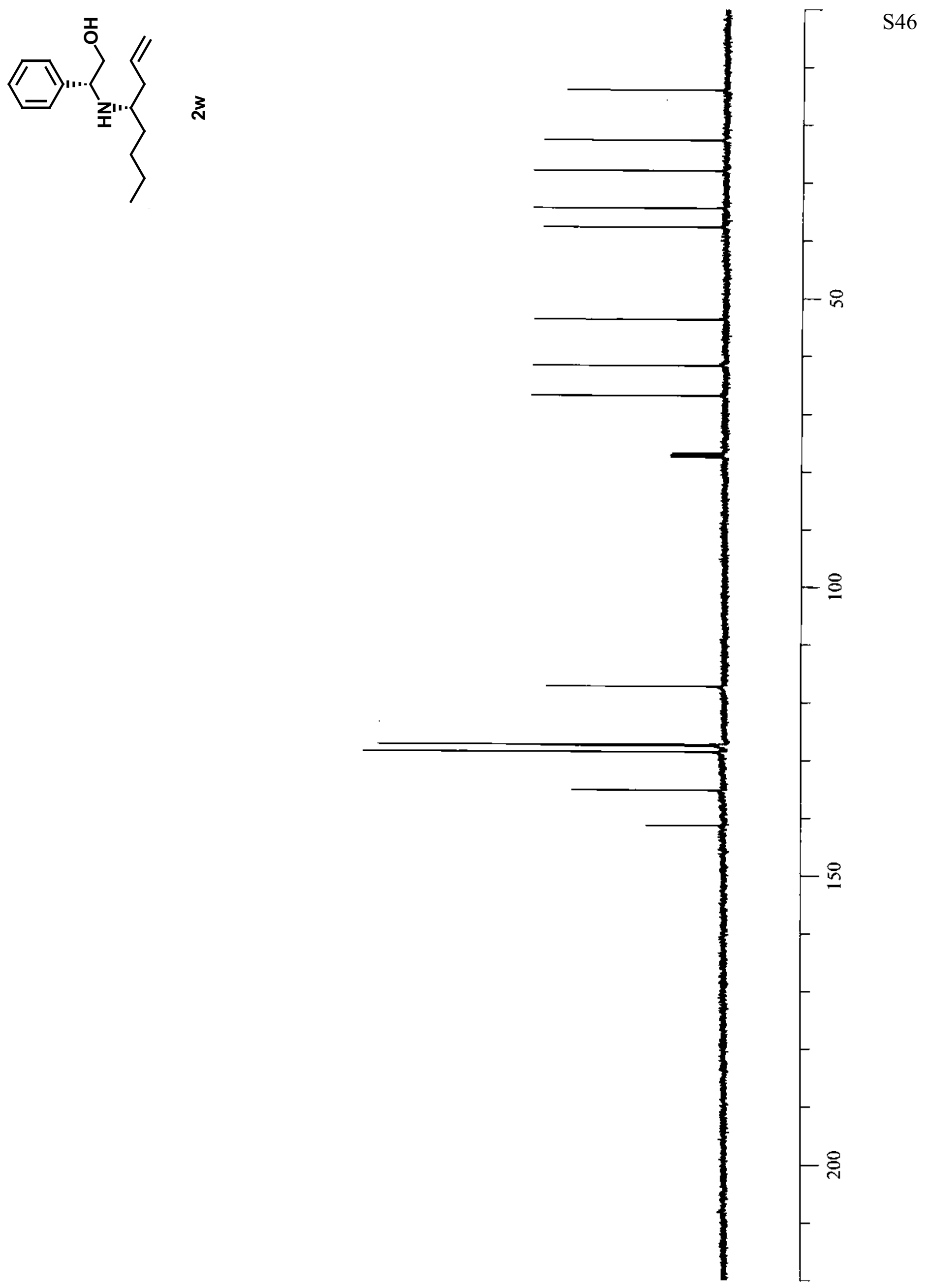

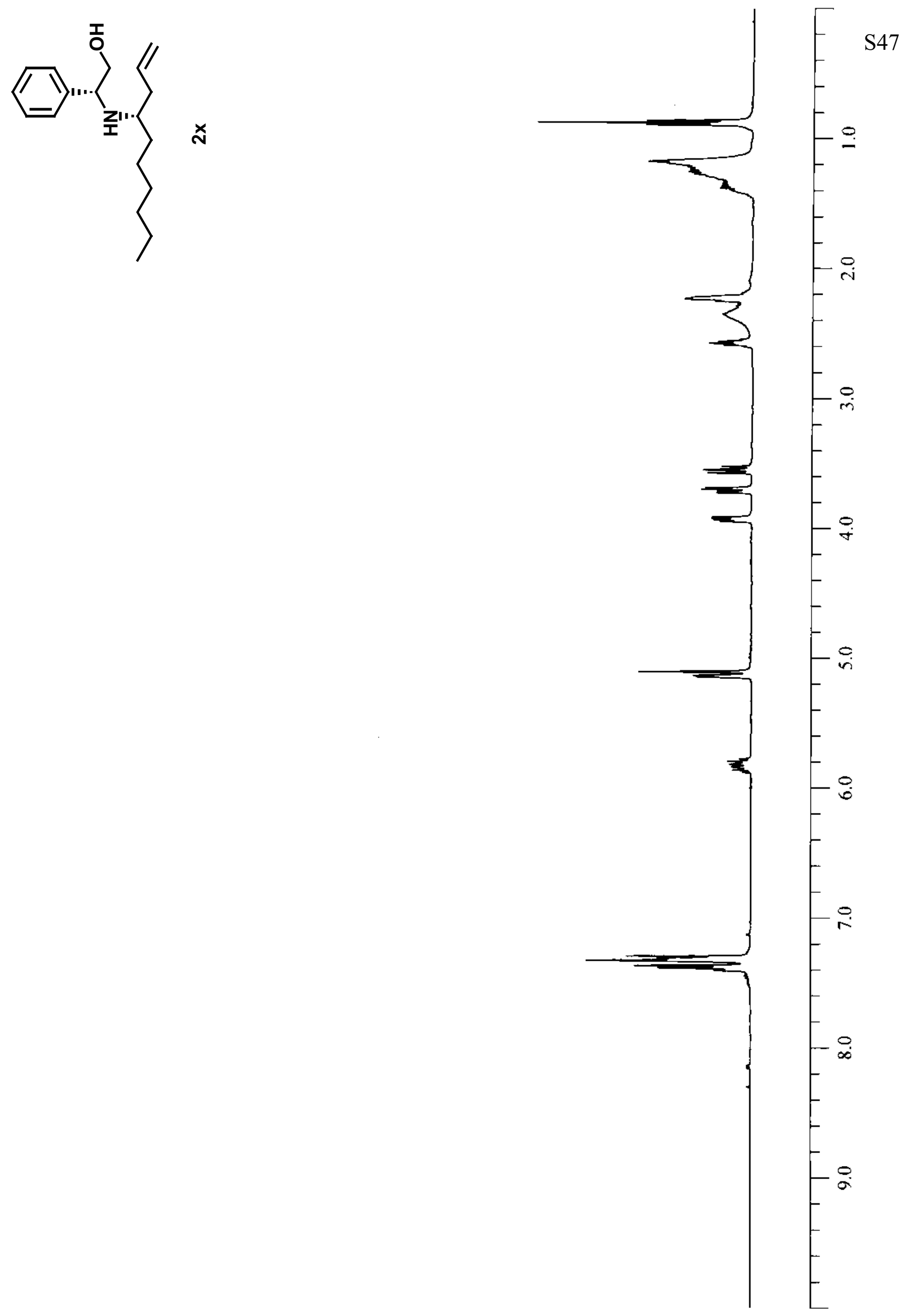

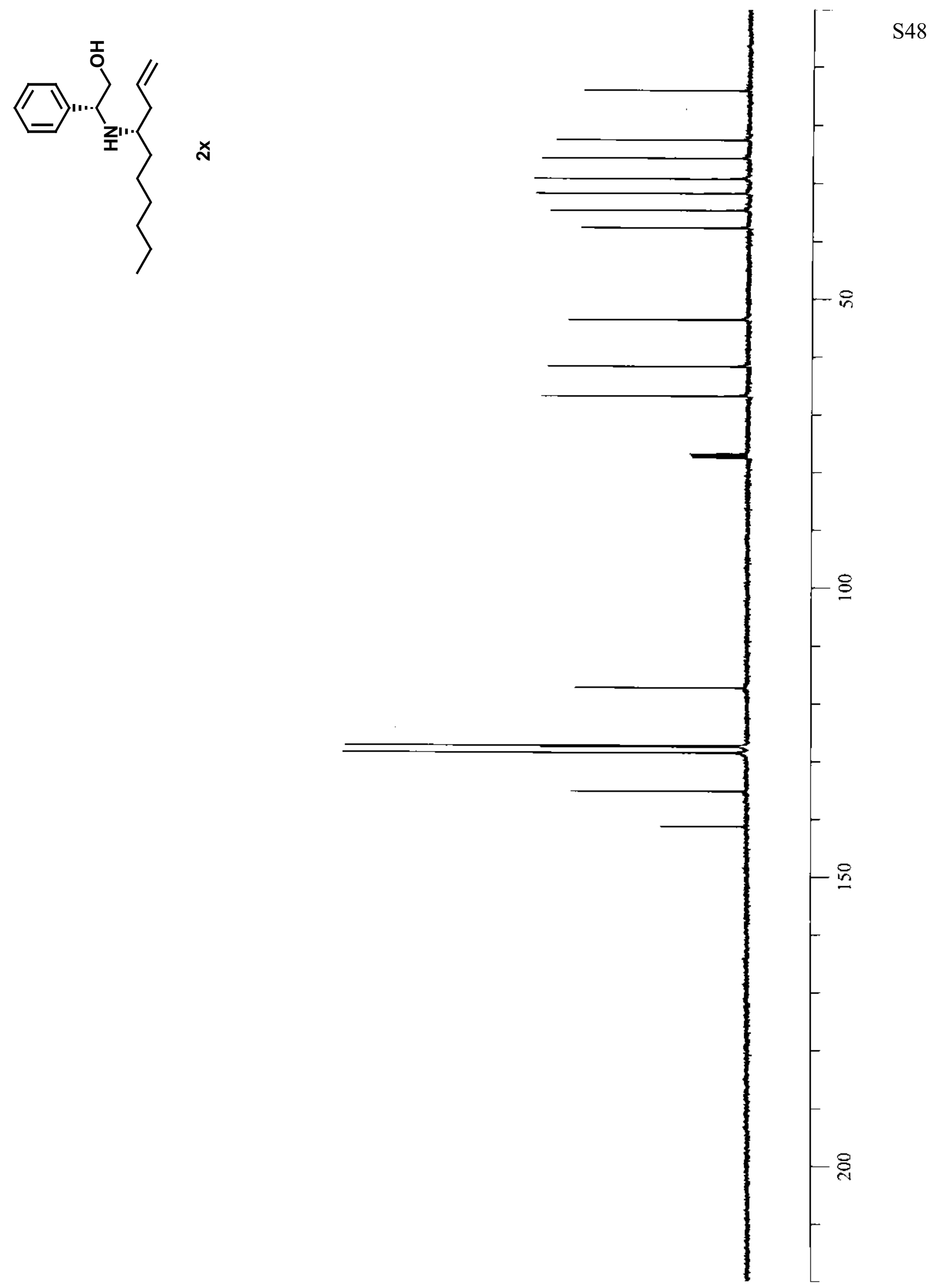

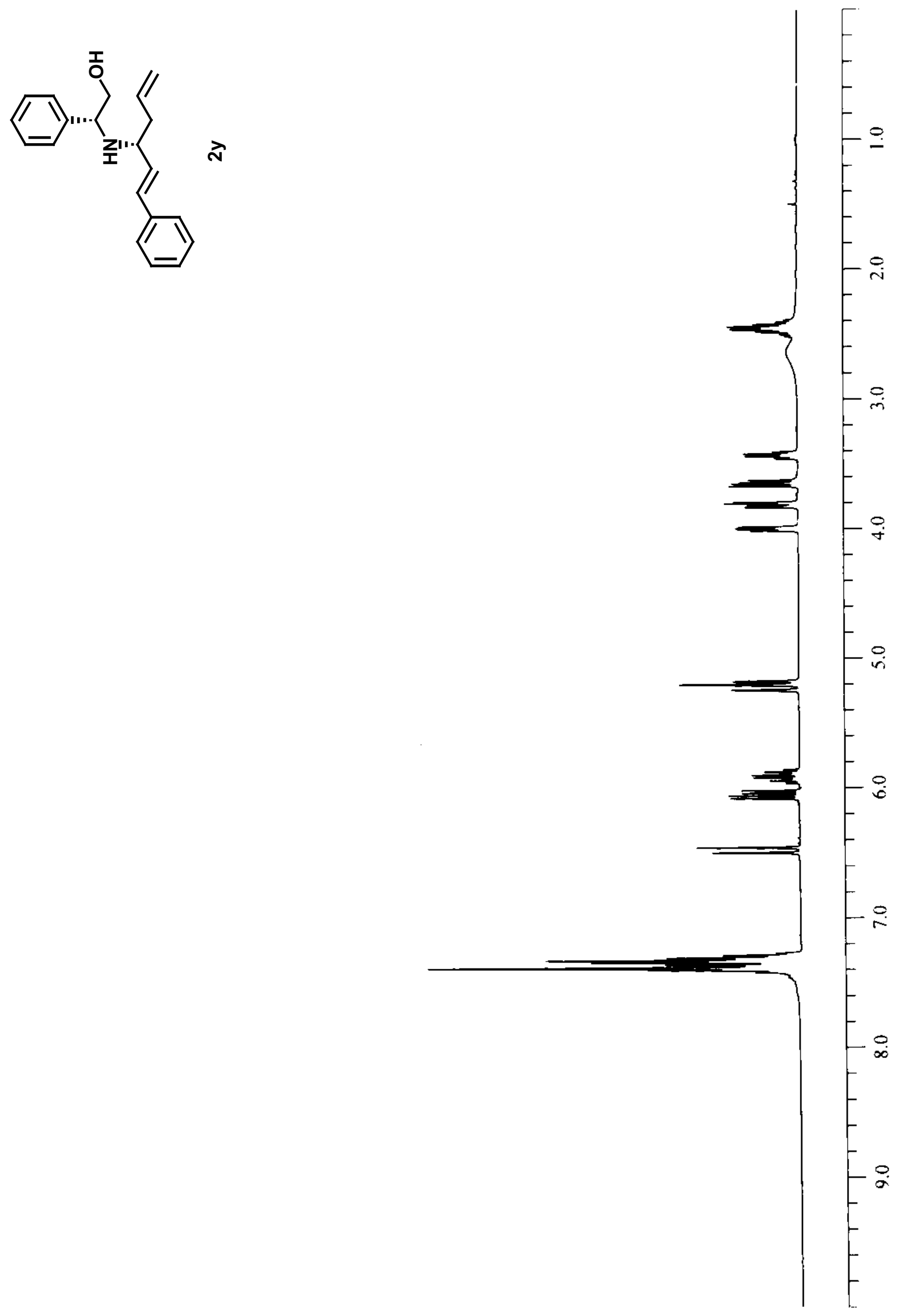

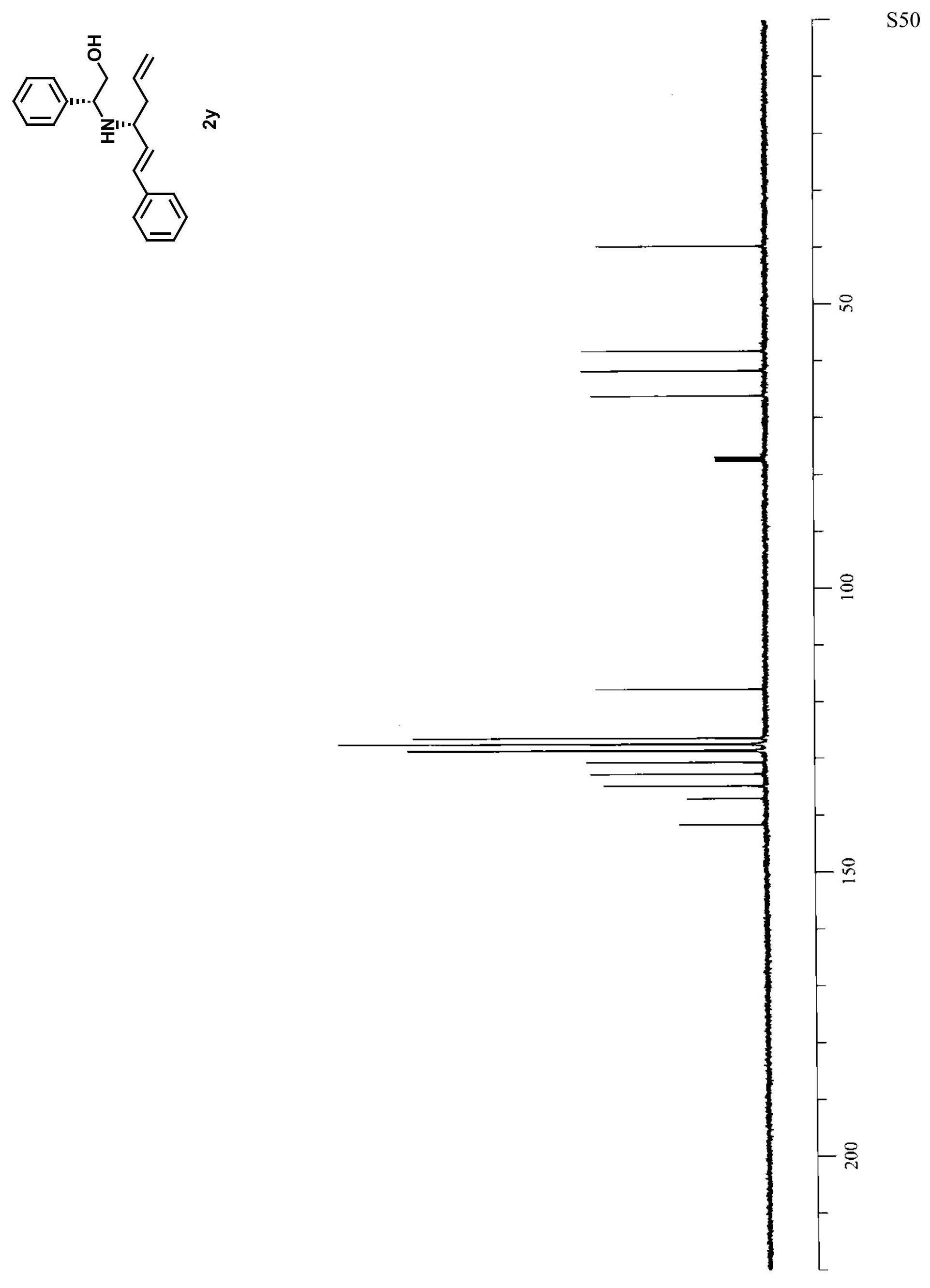

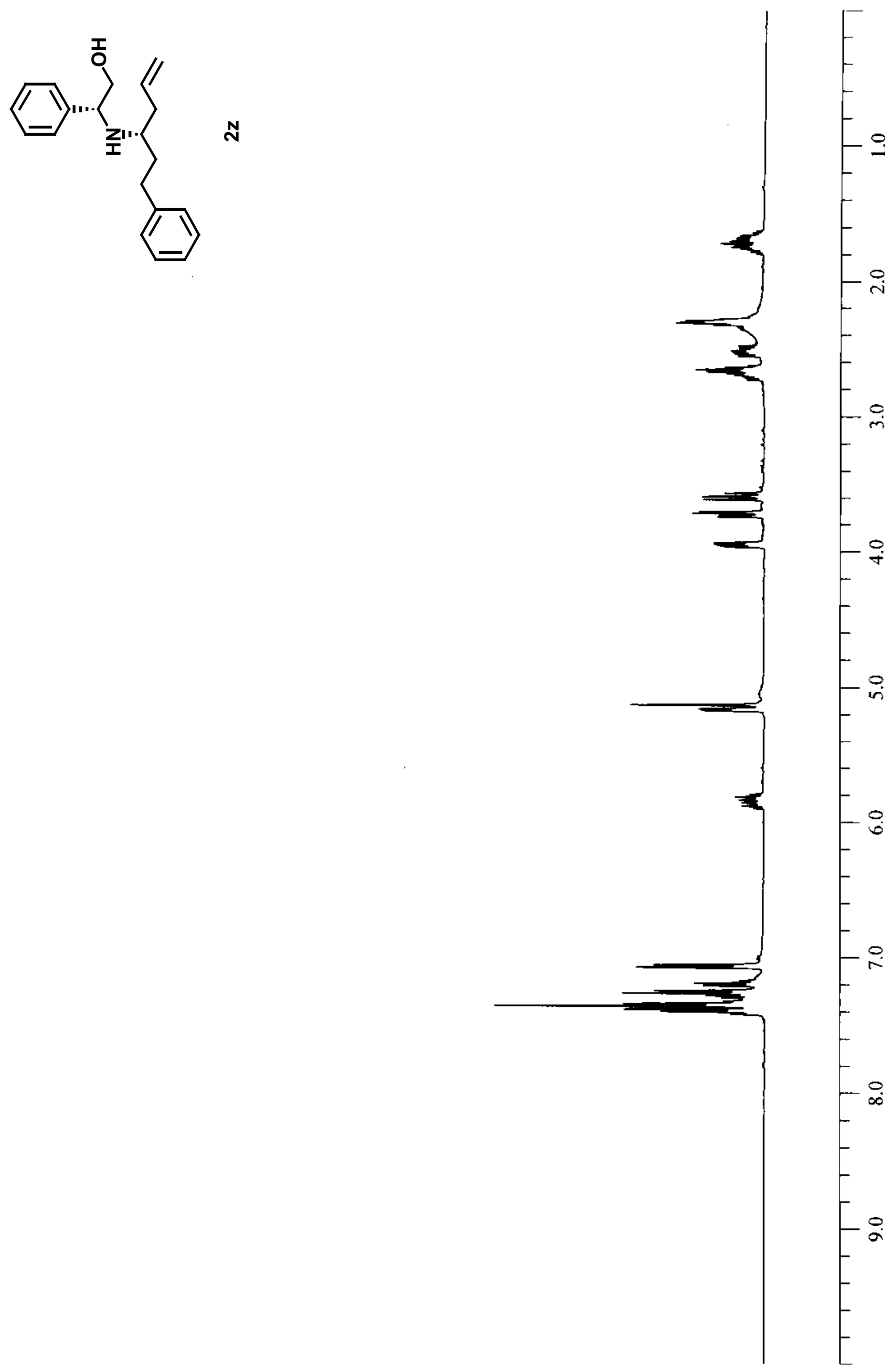

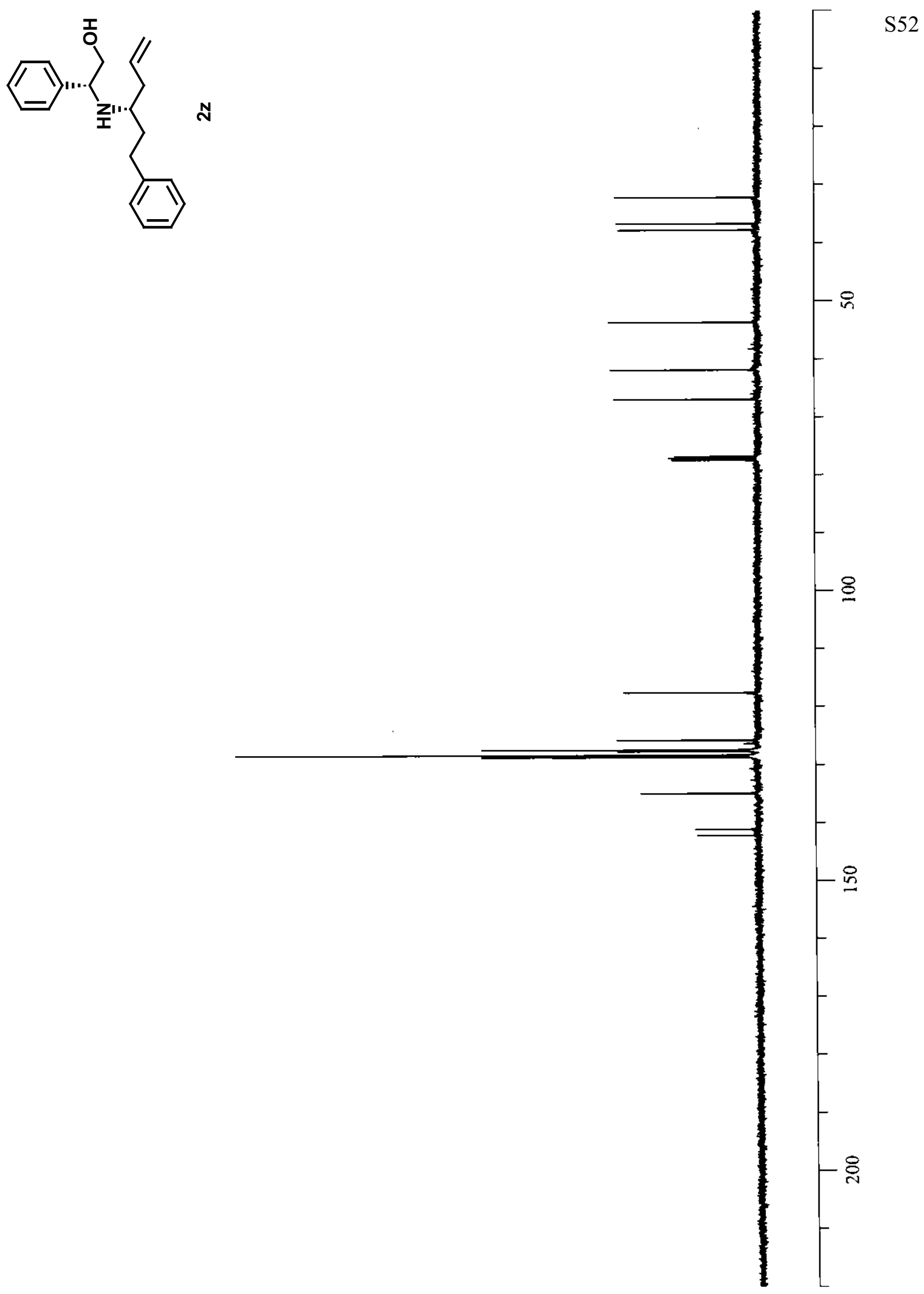

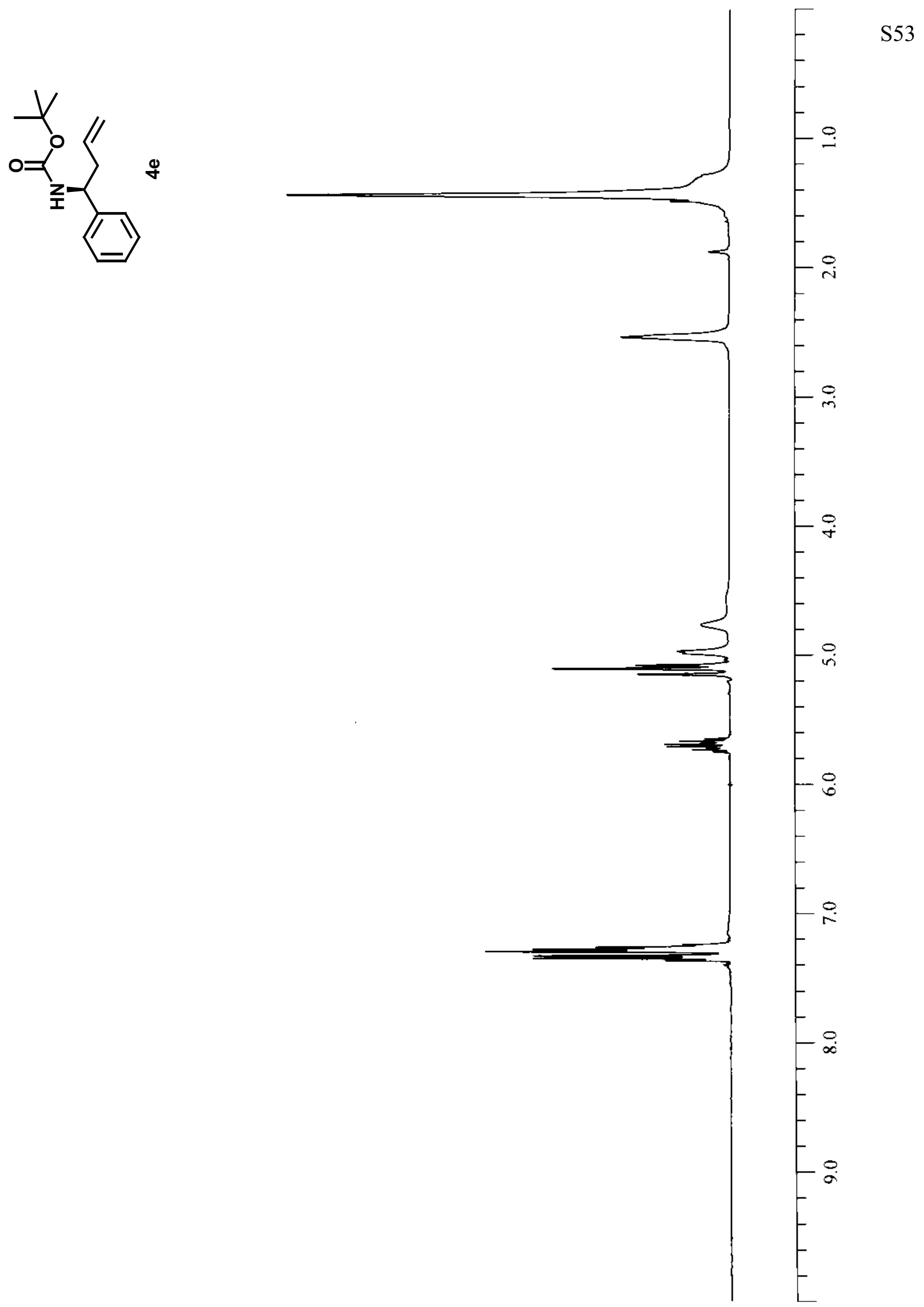

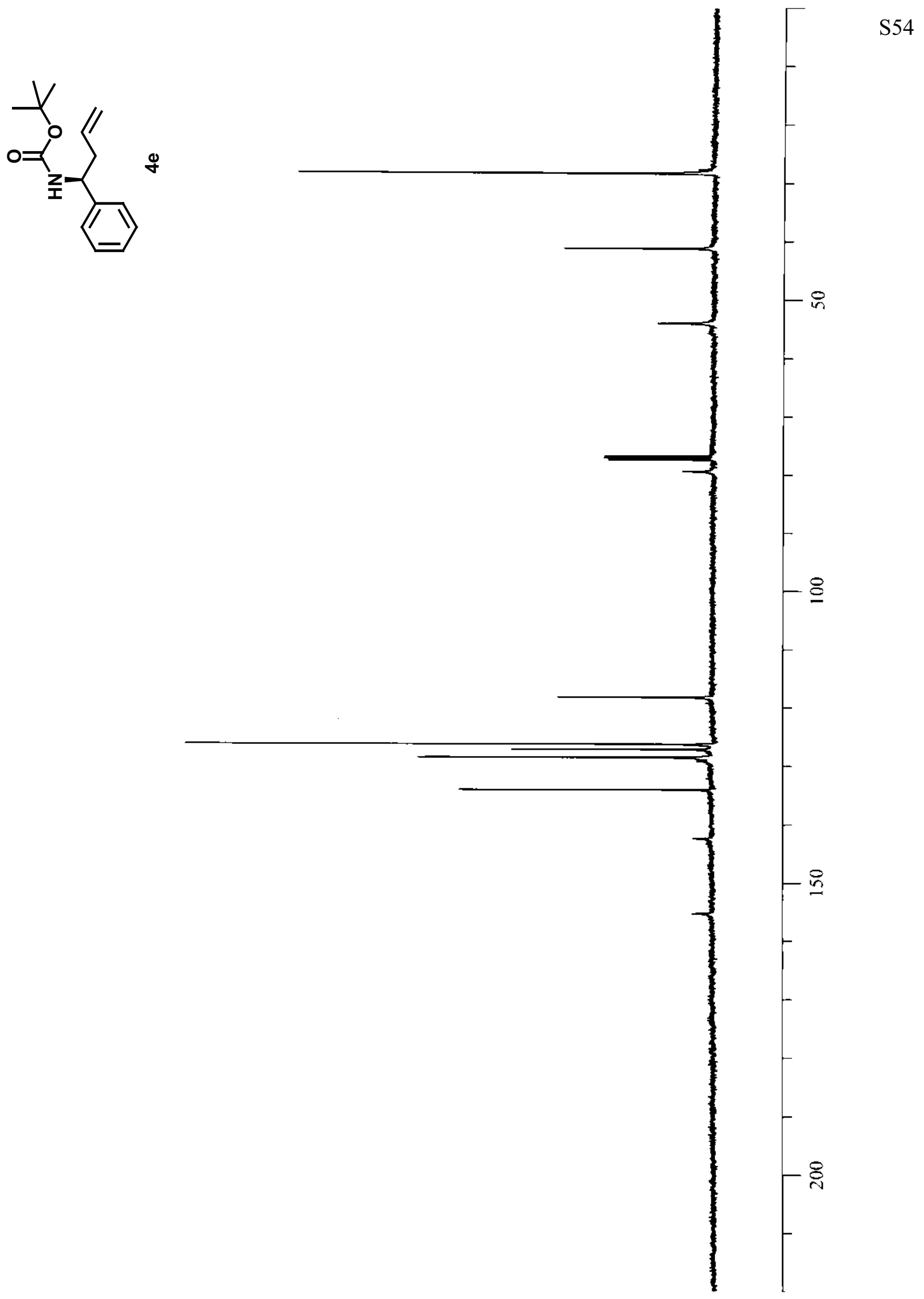

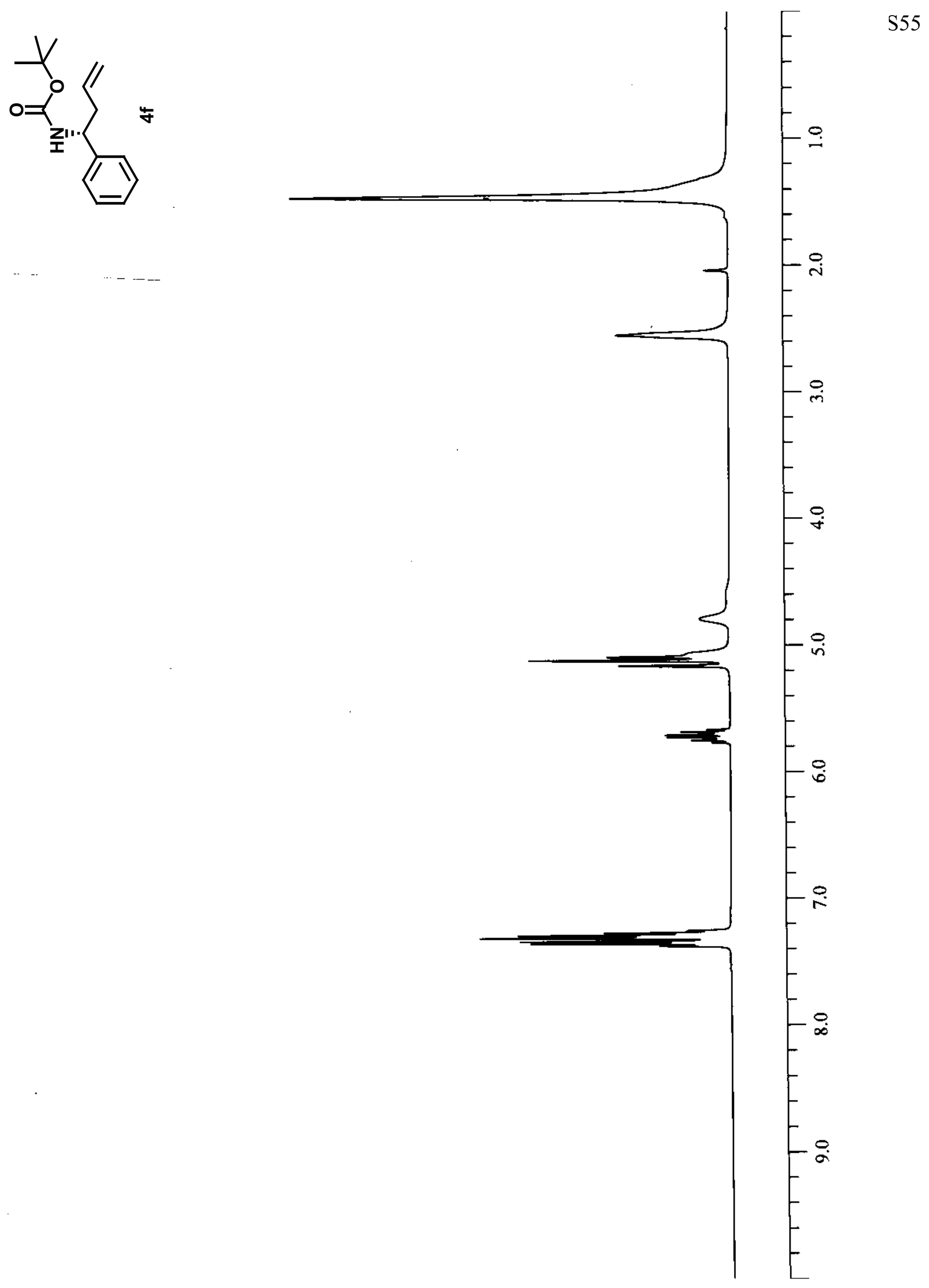

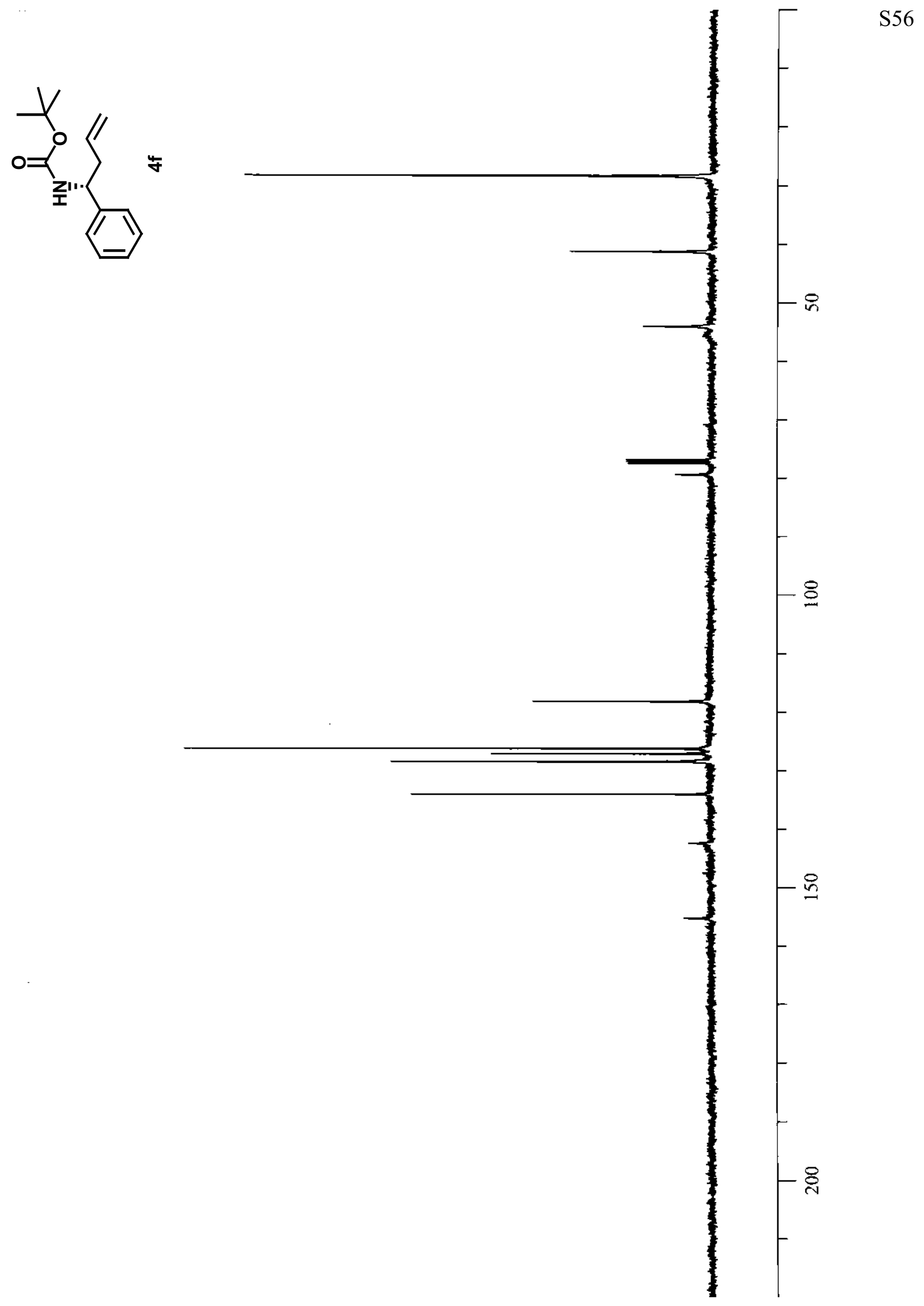

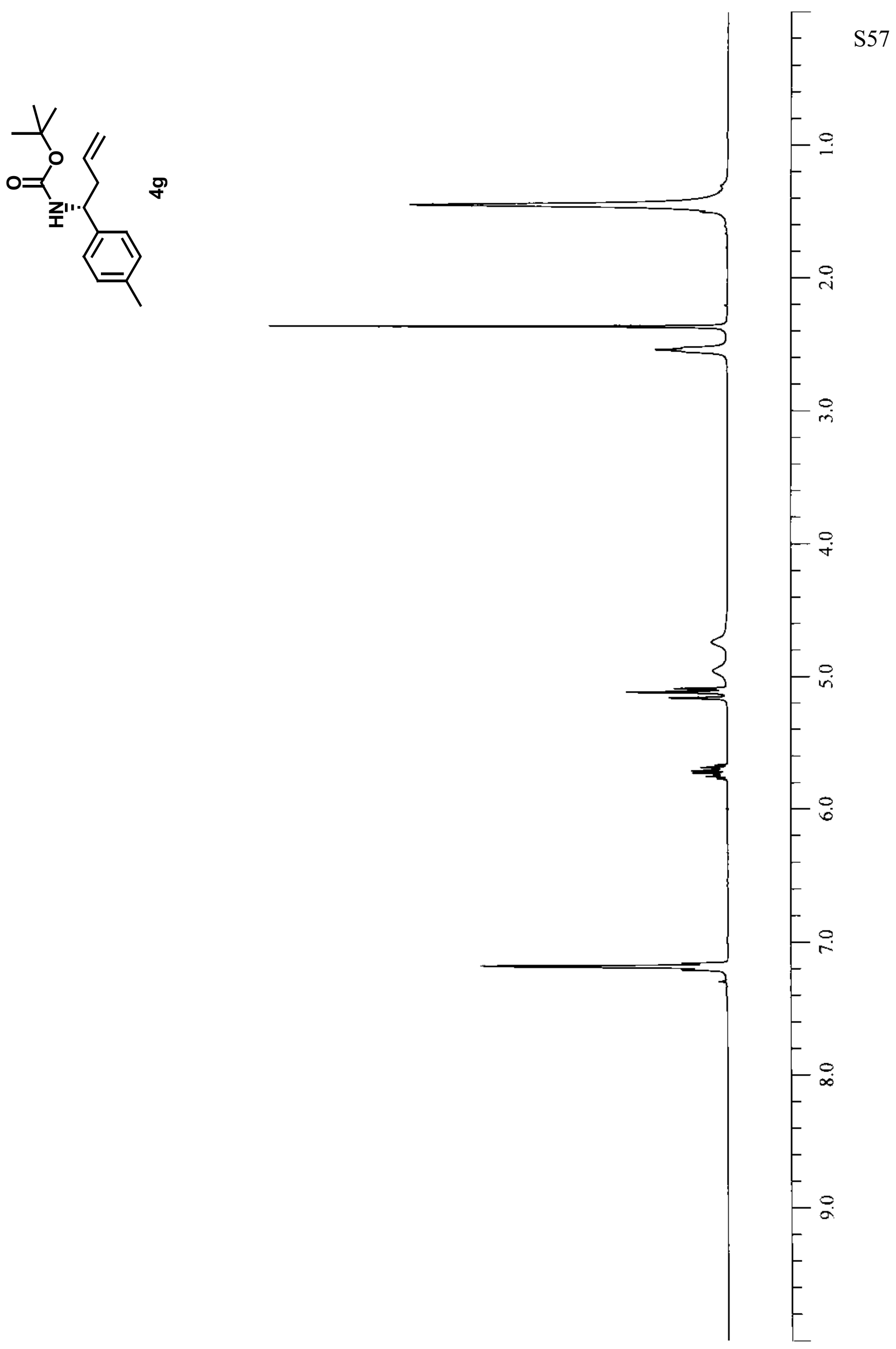


$$
9
$$



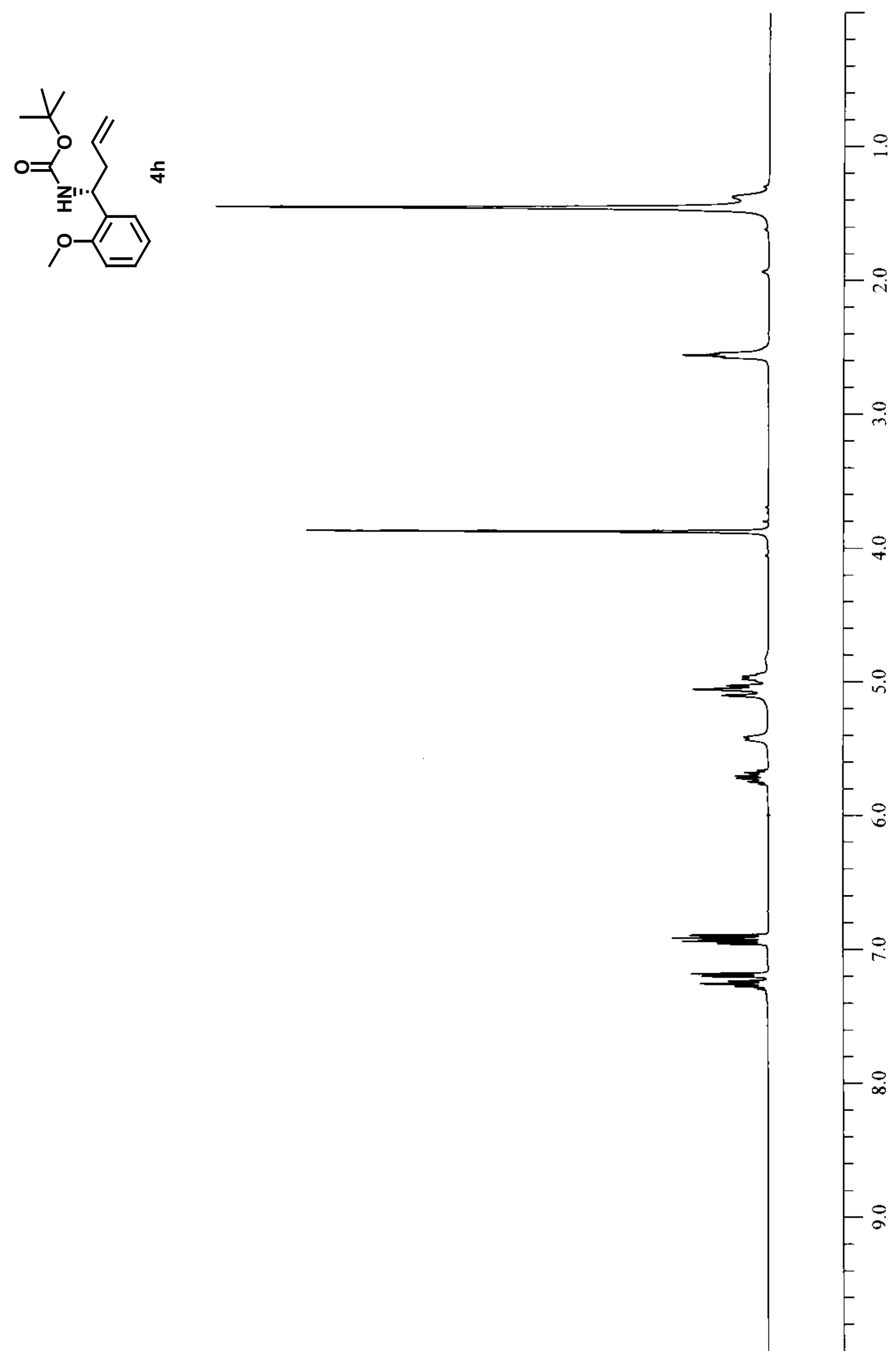

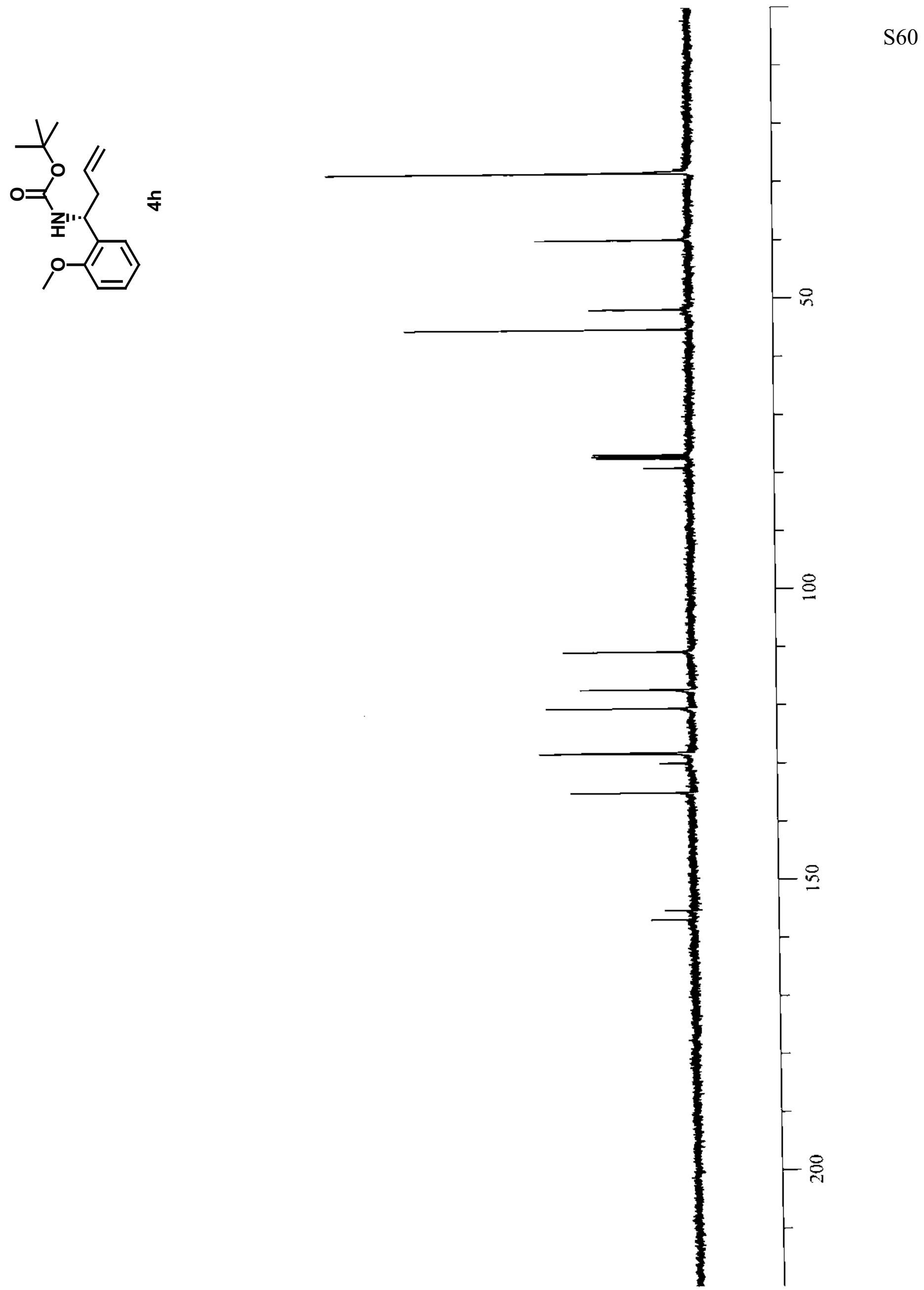

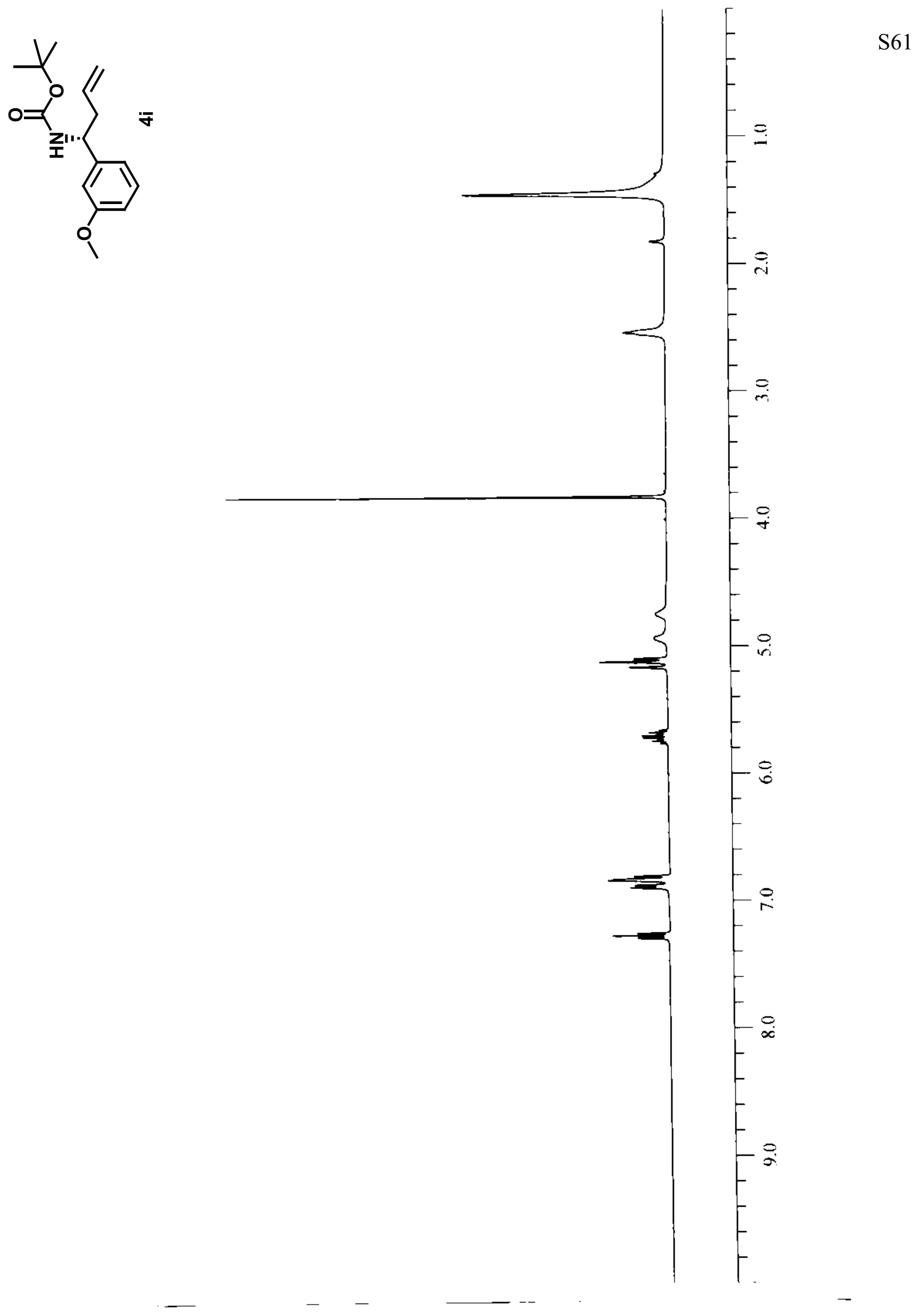

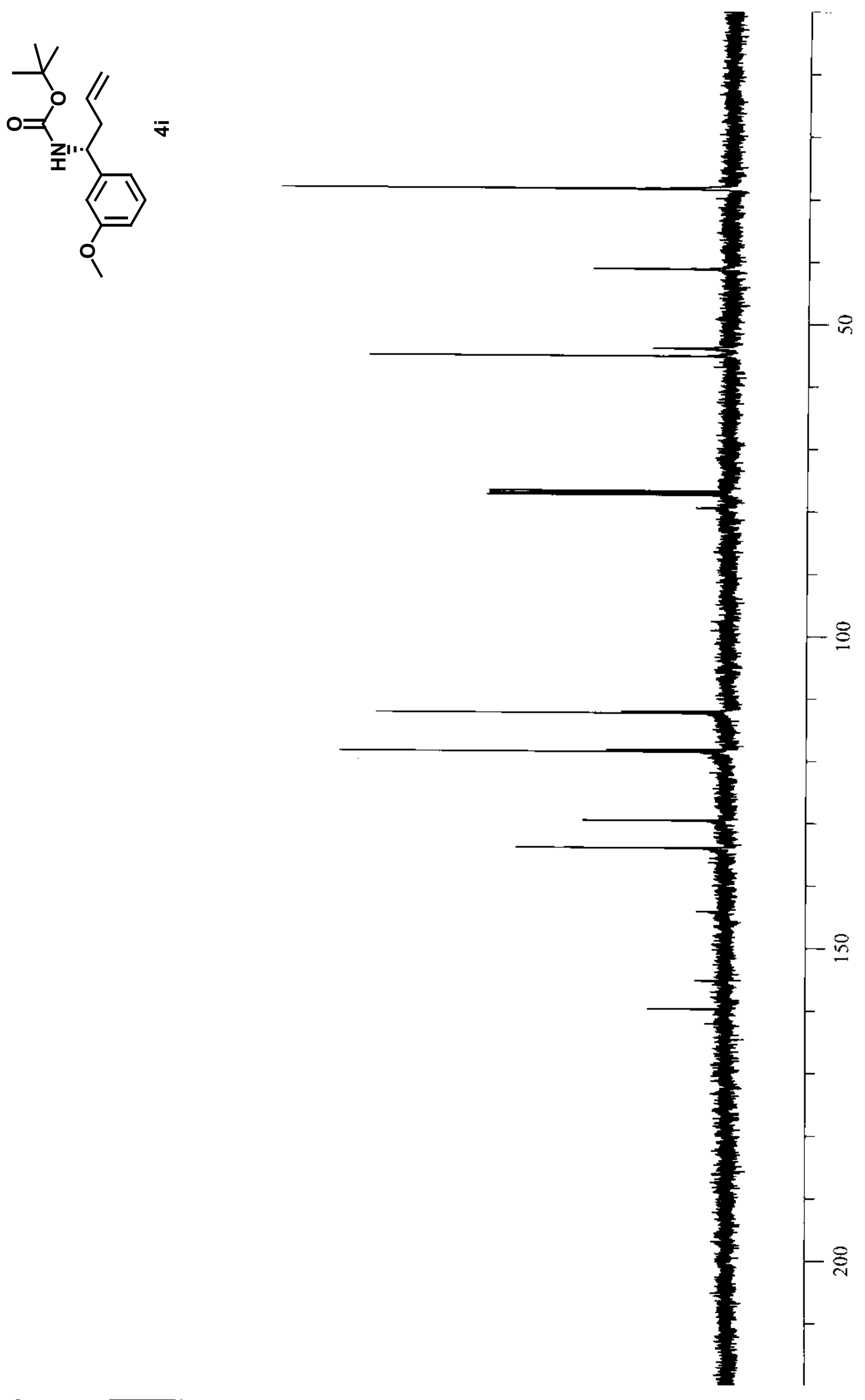

S62 

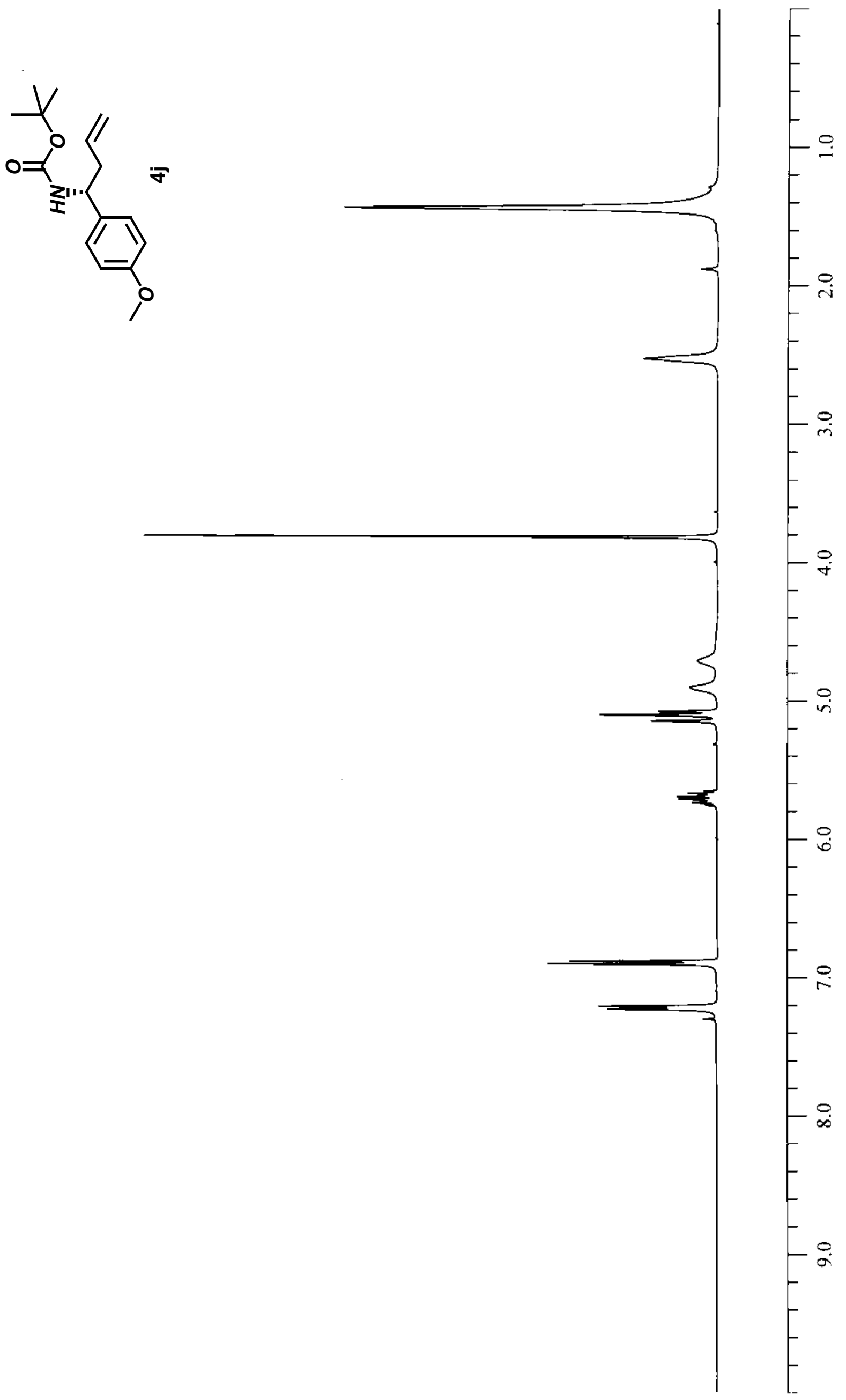

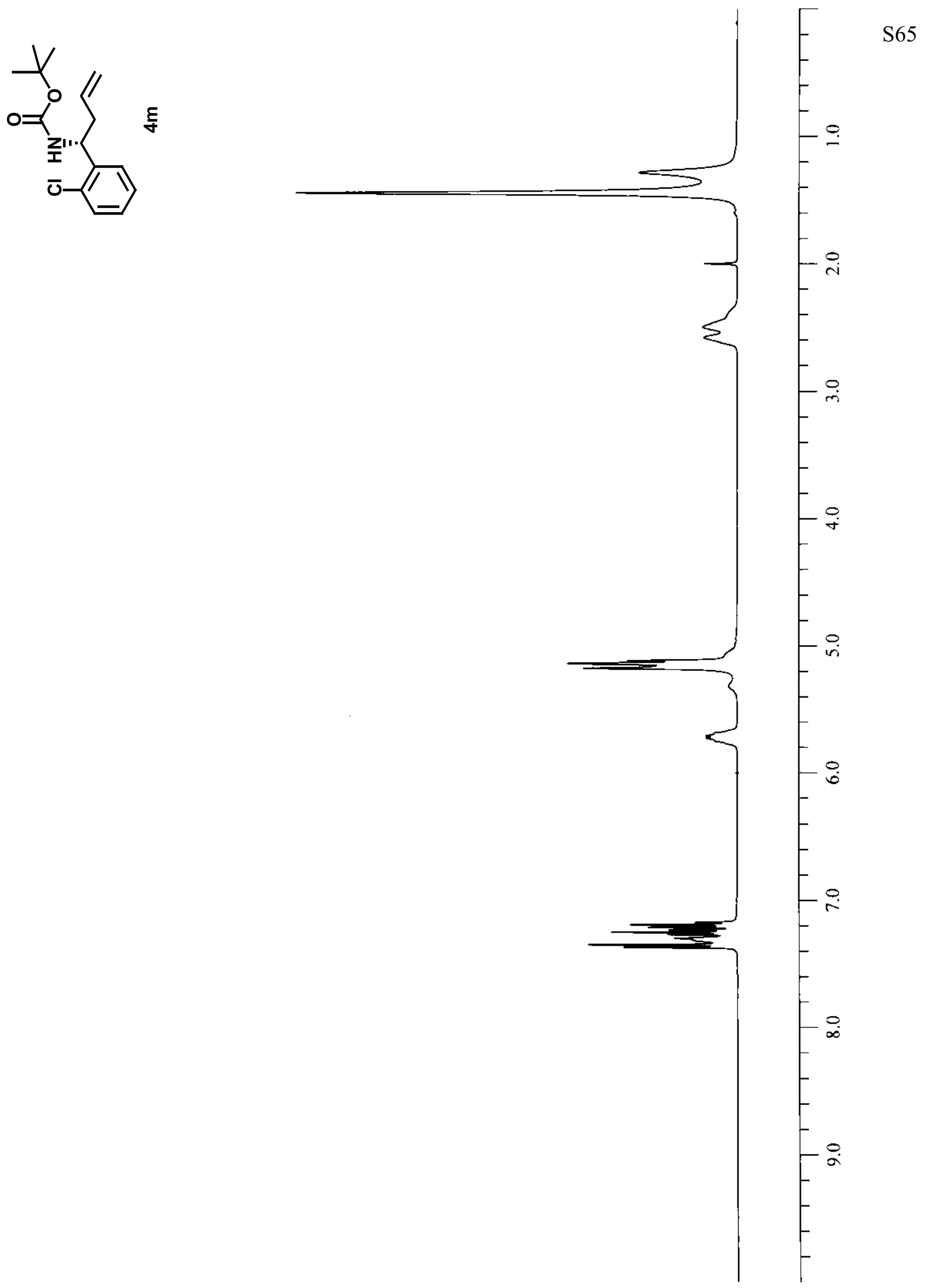
$V_{2}$
$0=1$
$0=1$
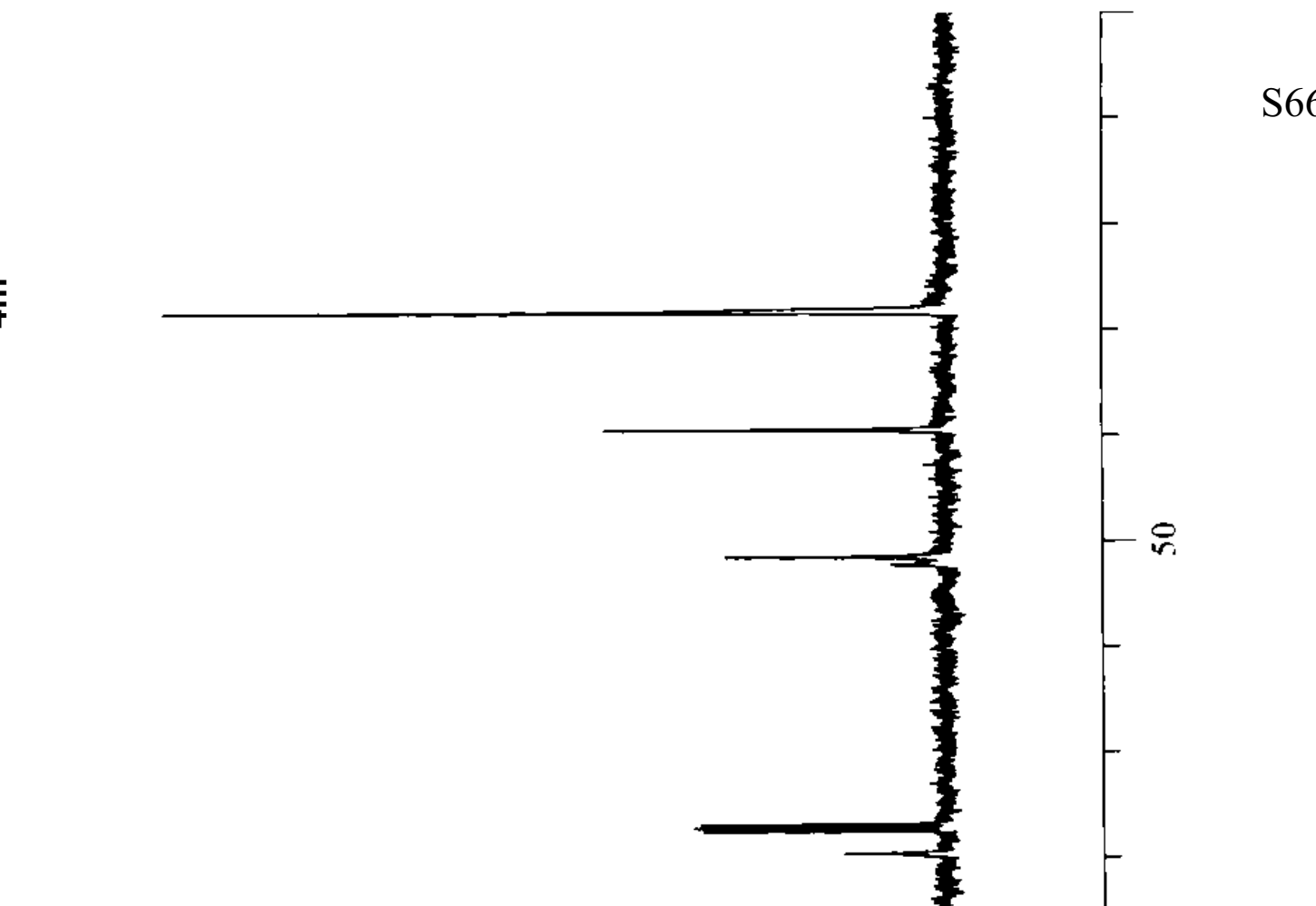

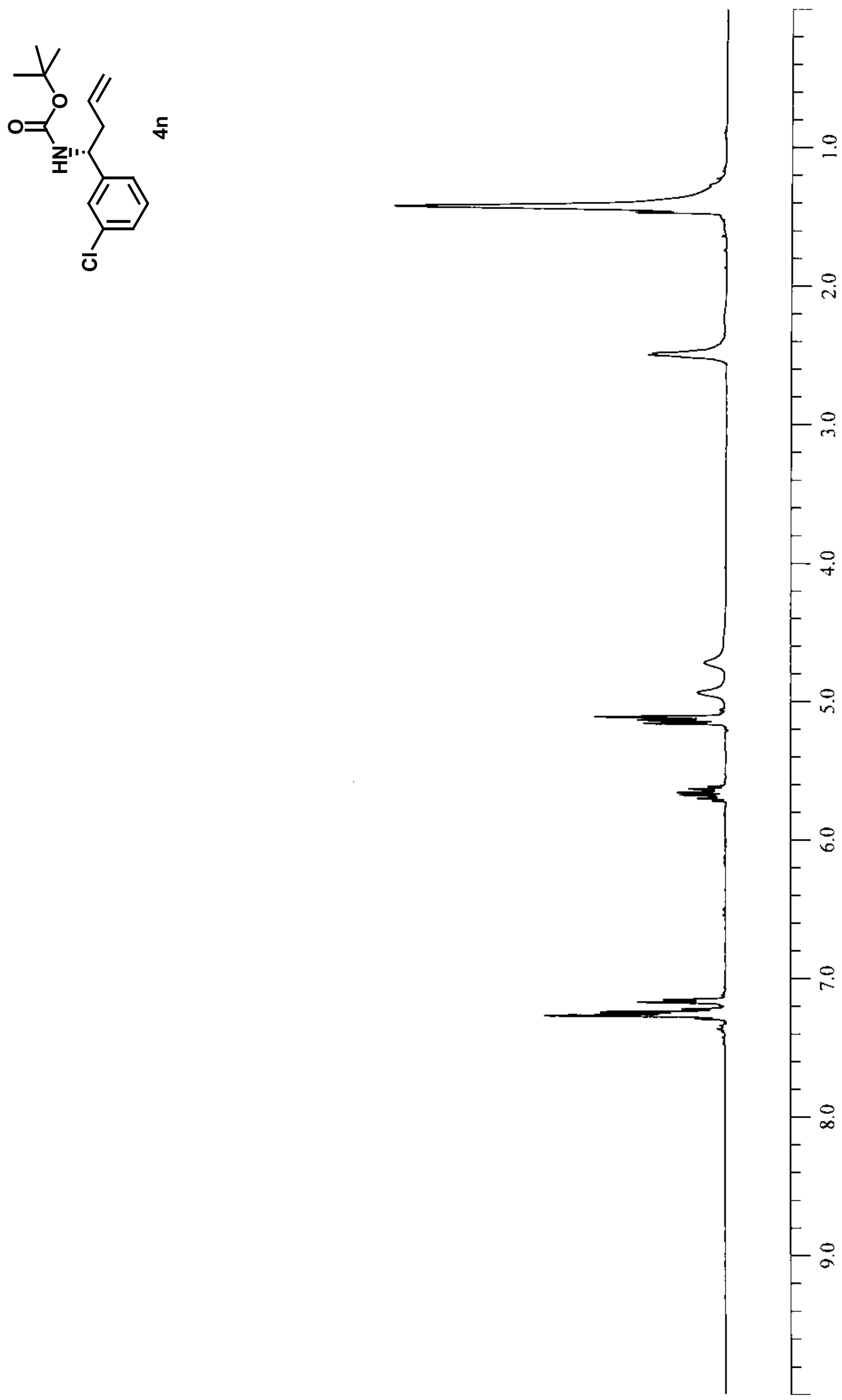


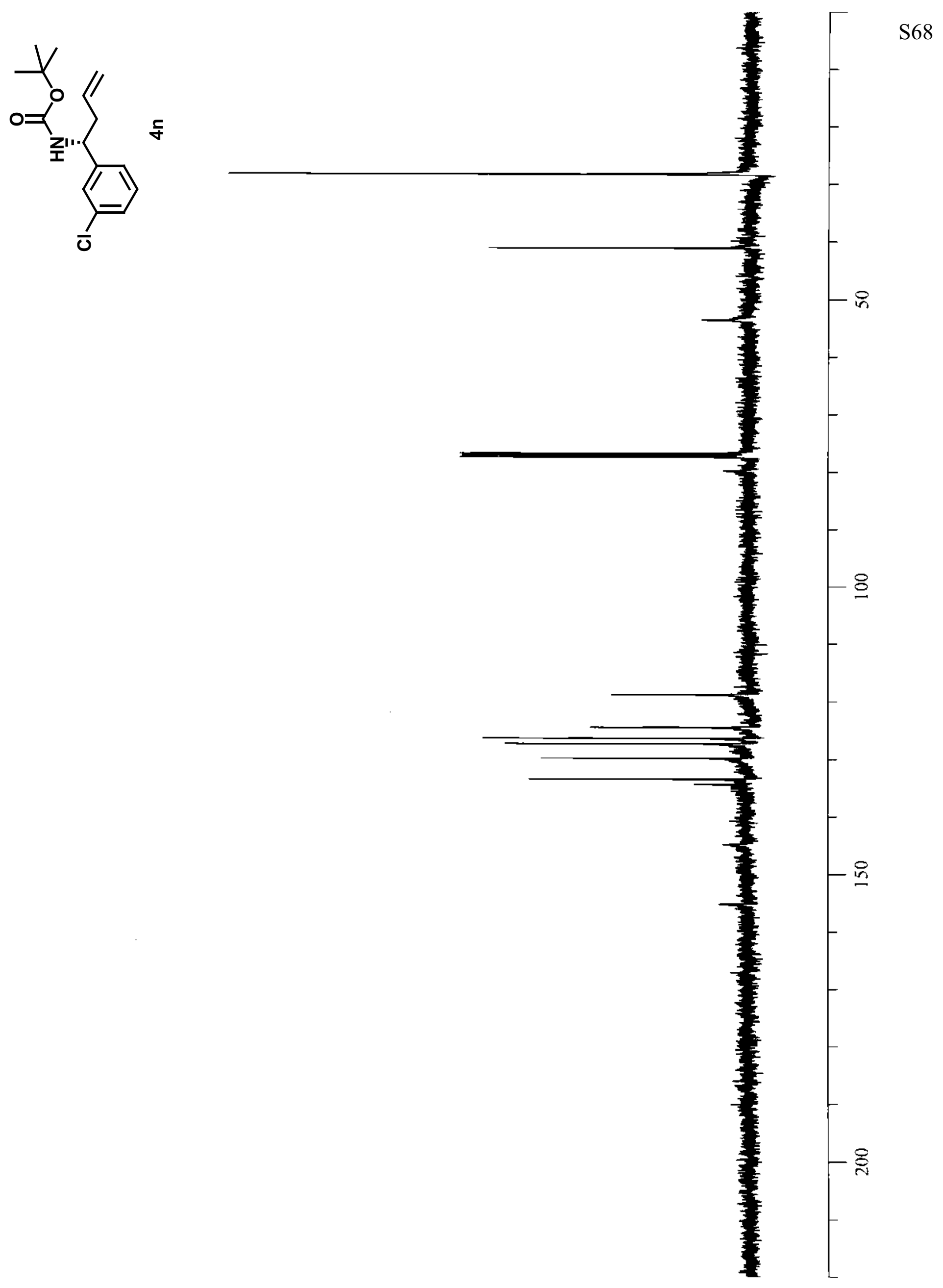



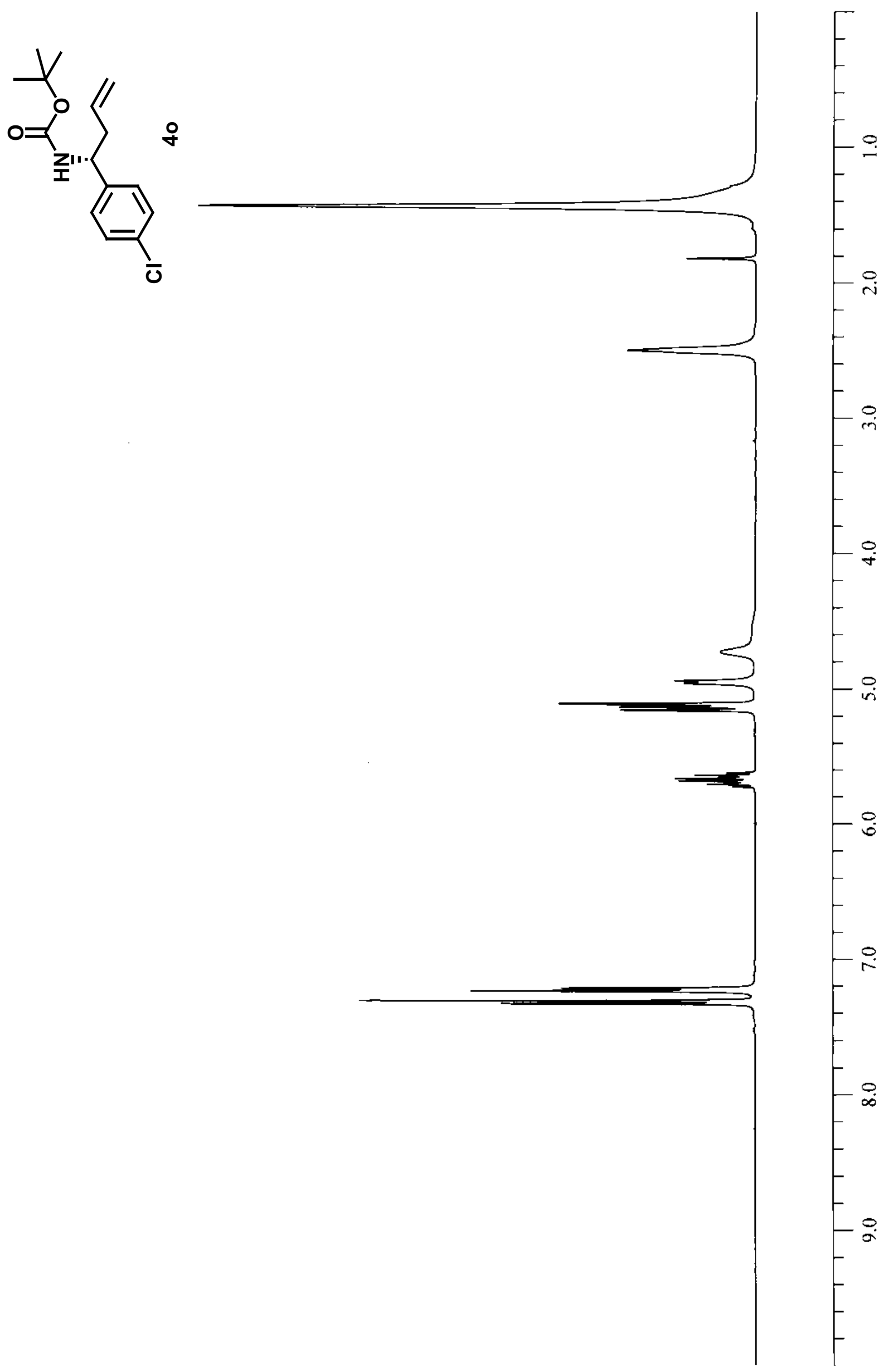

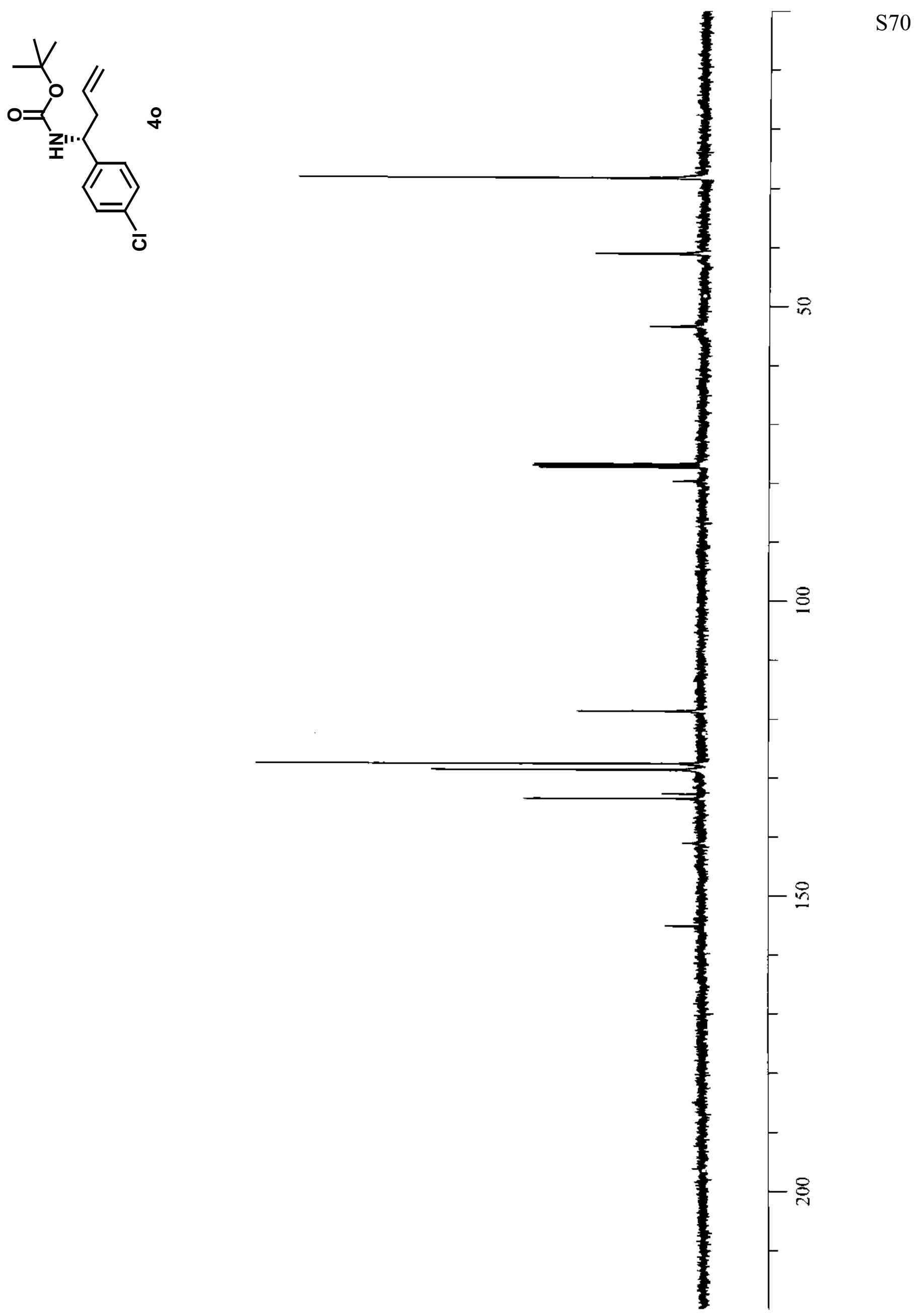

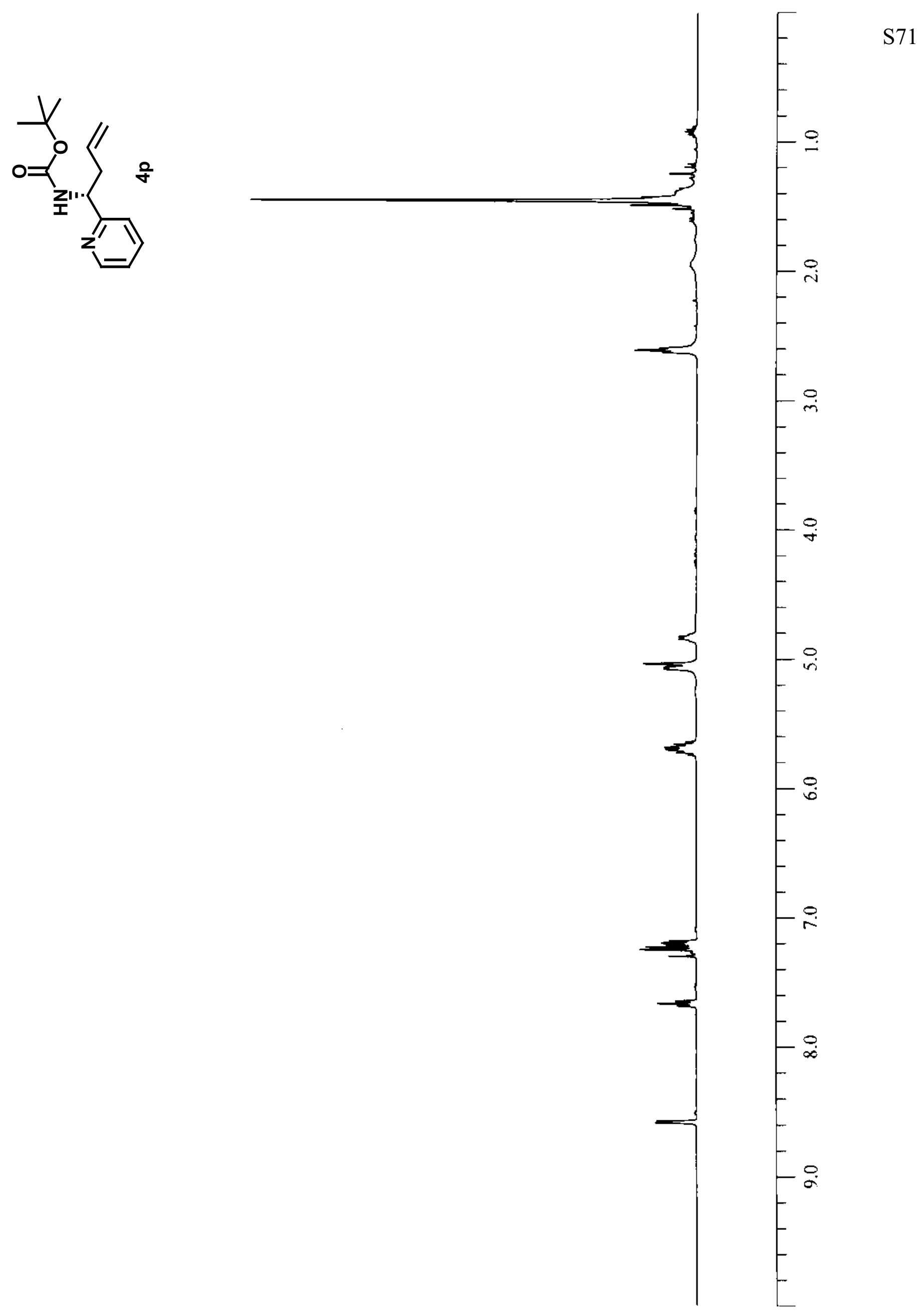


$$
1
$$




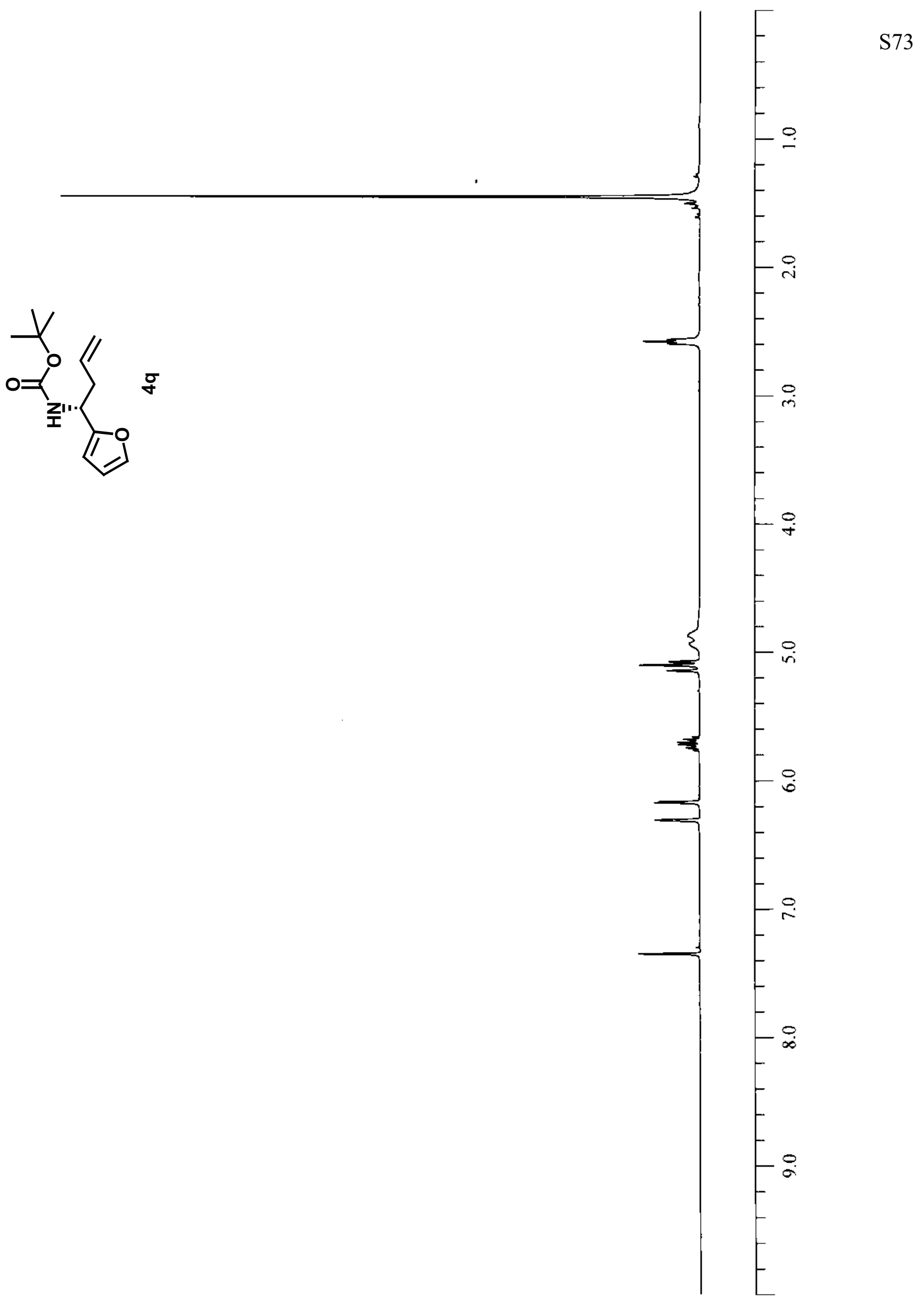




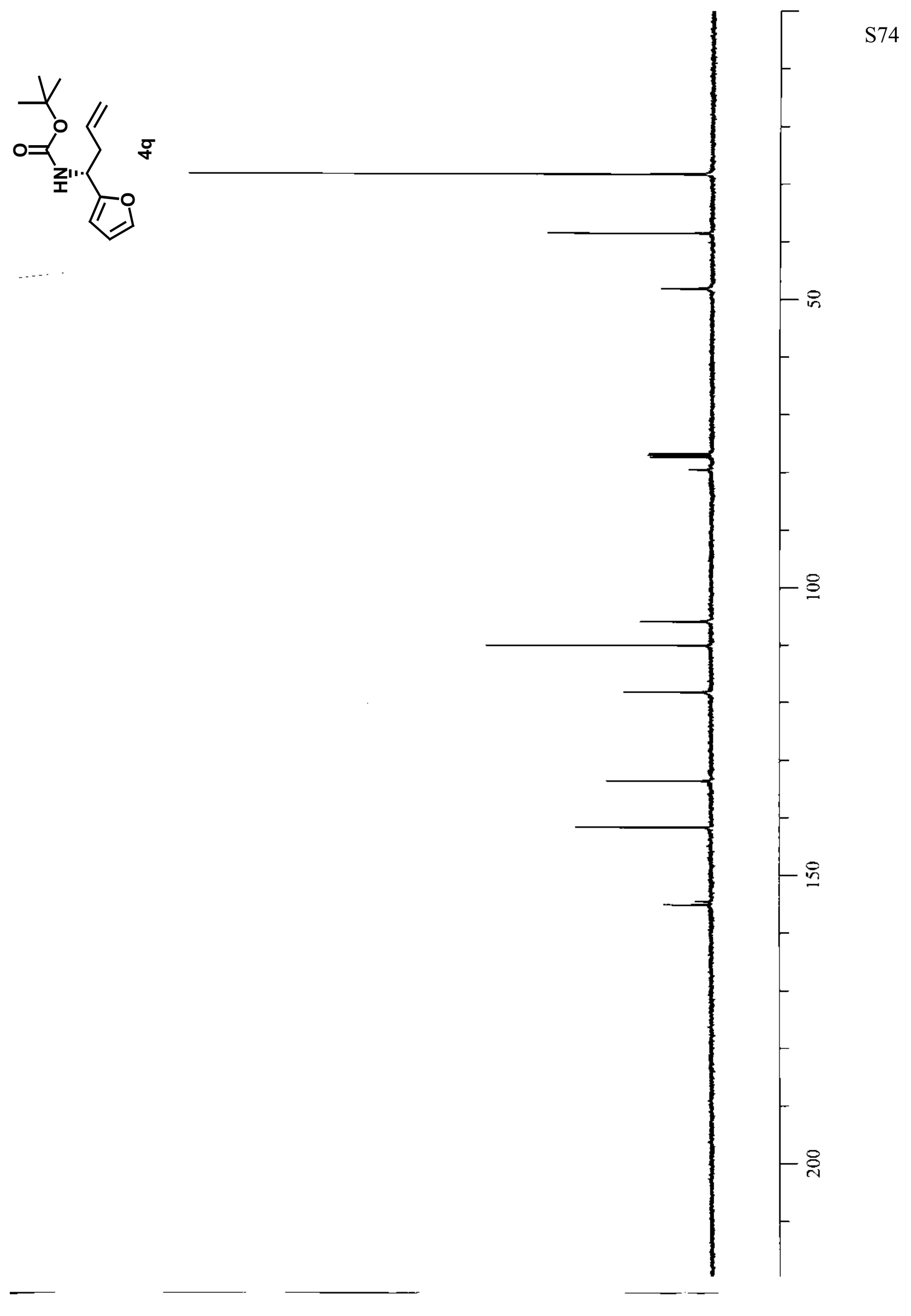




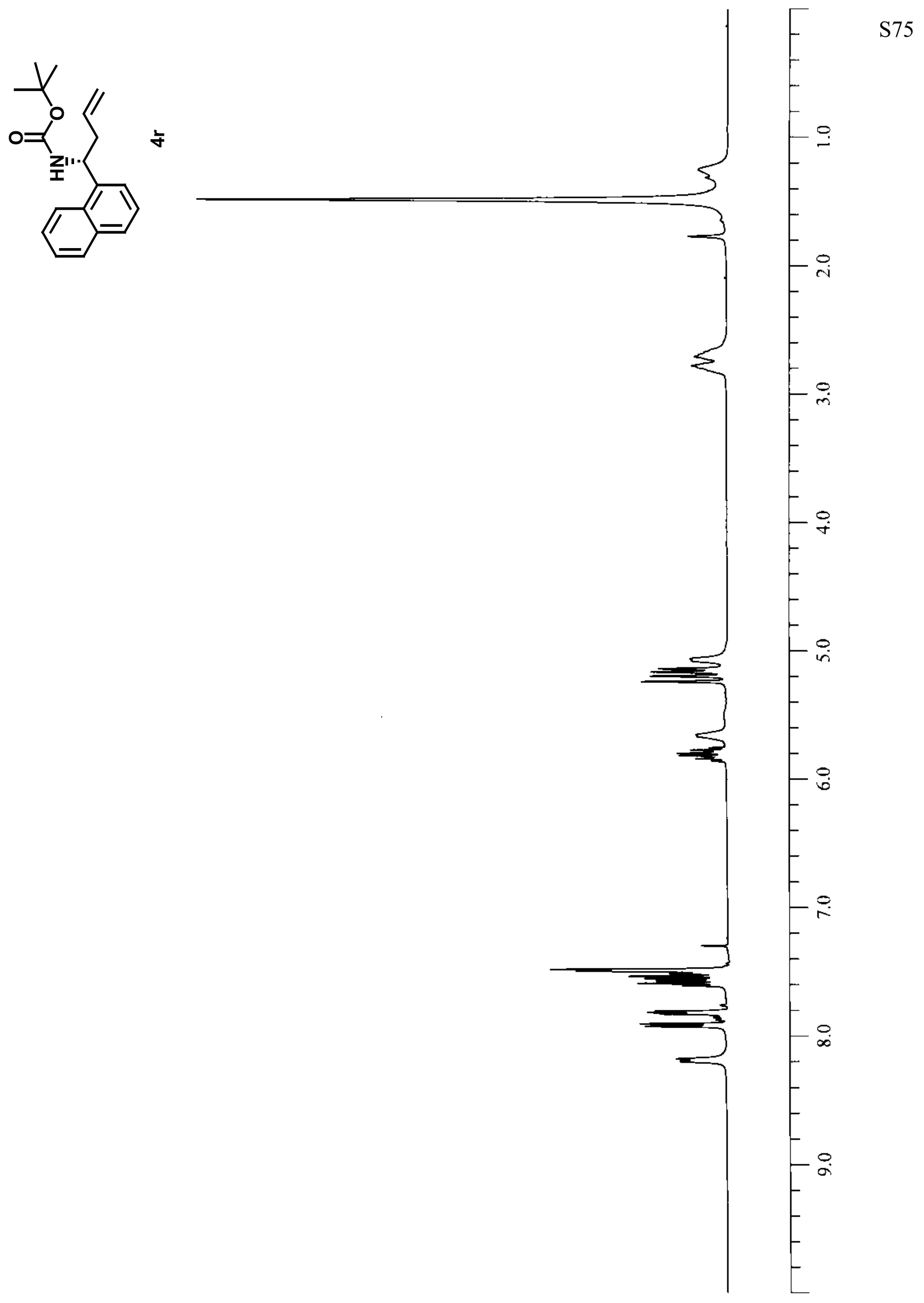



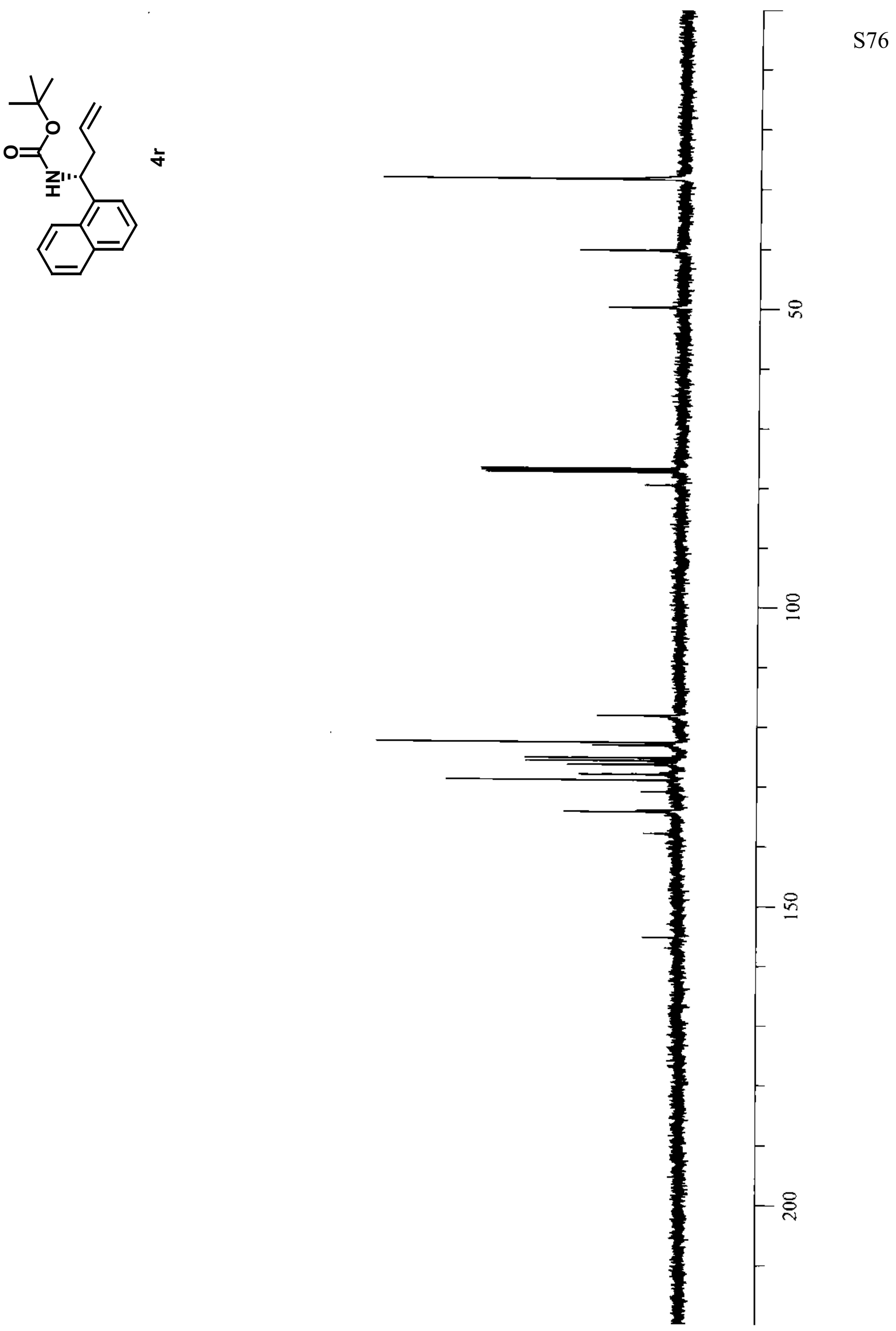


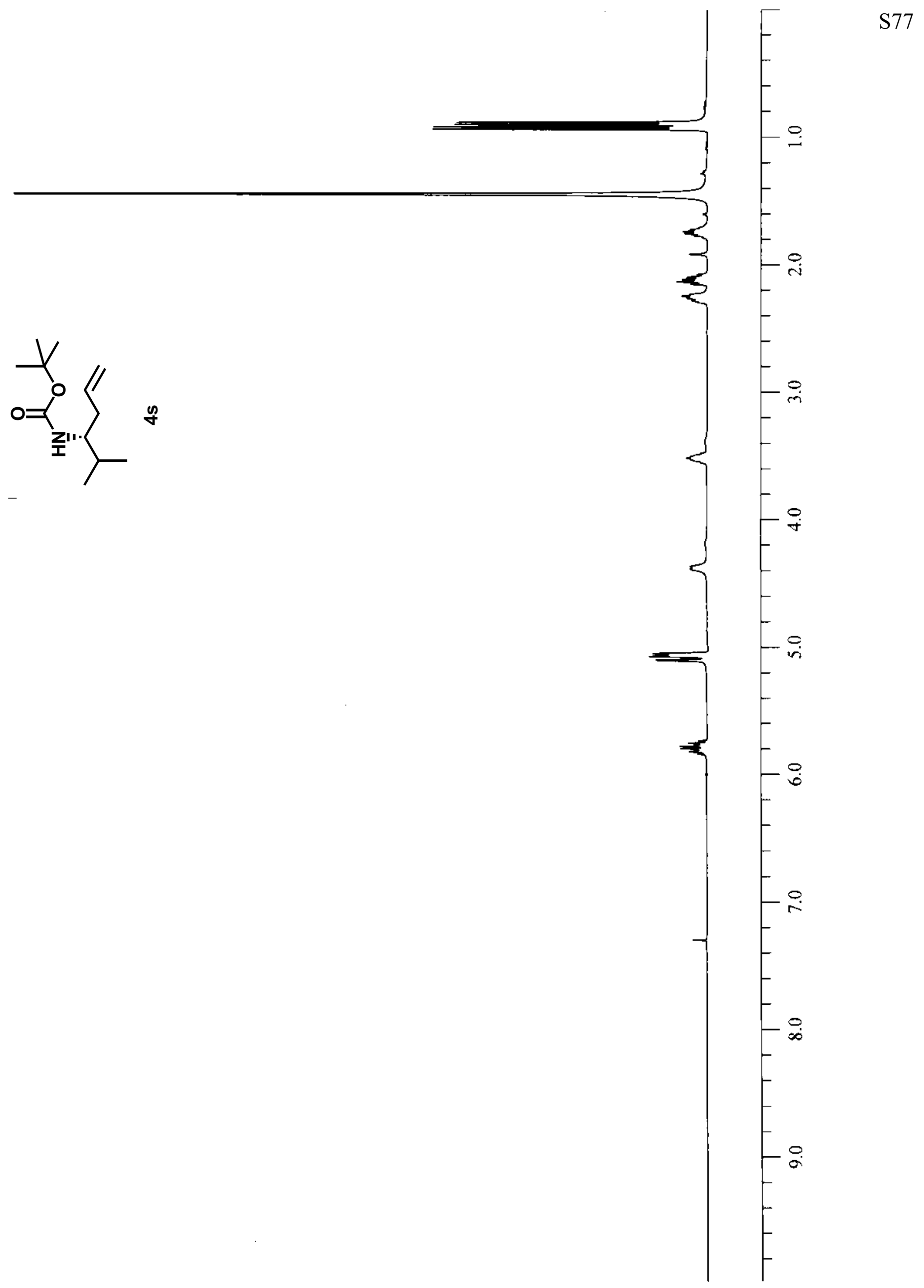




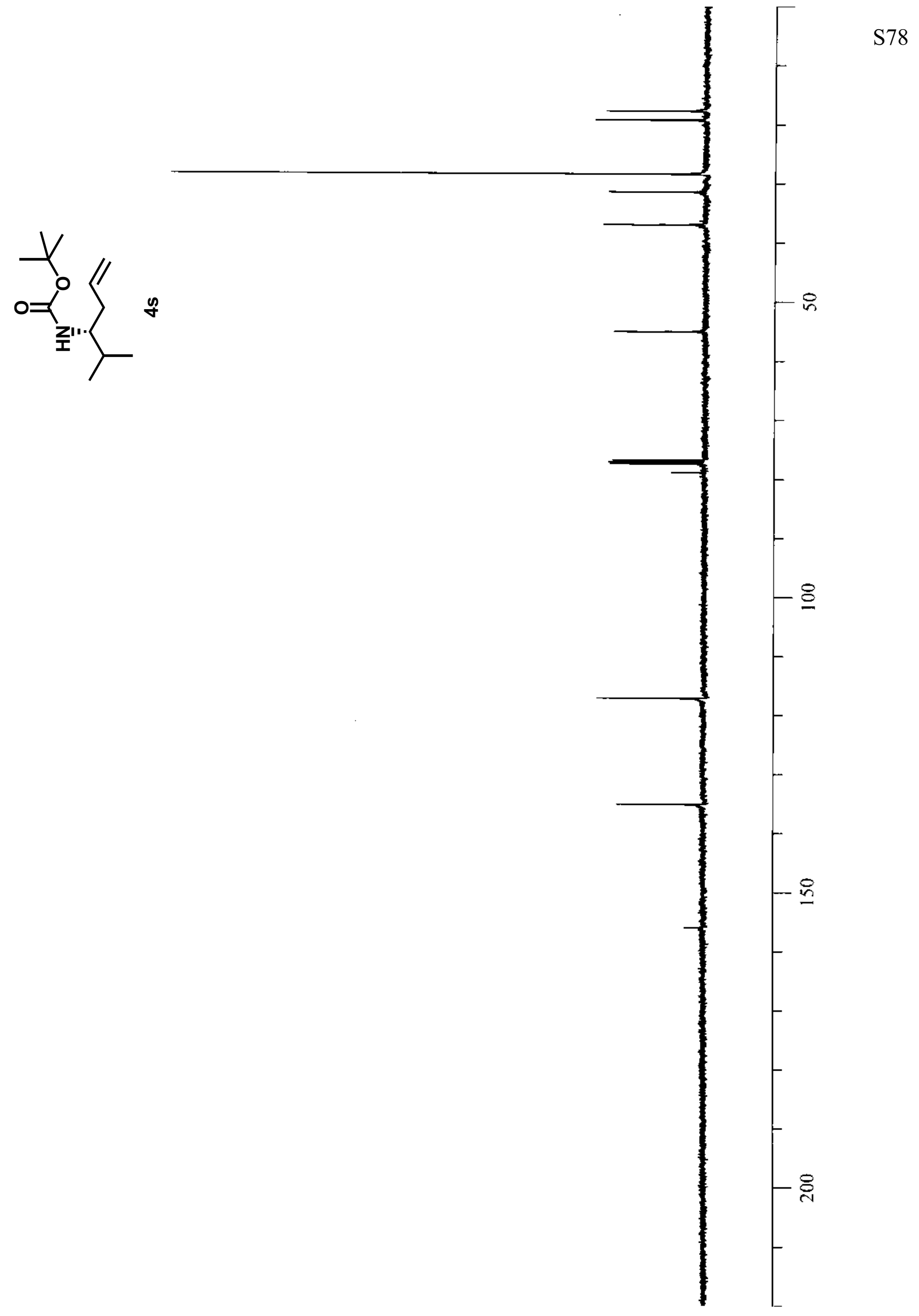




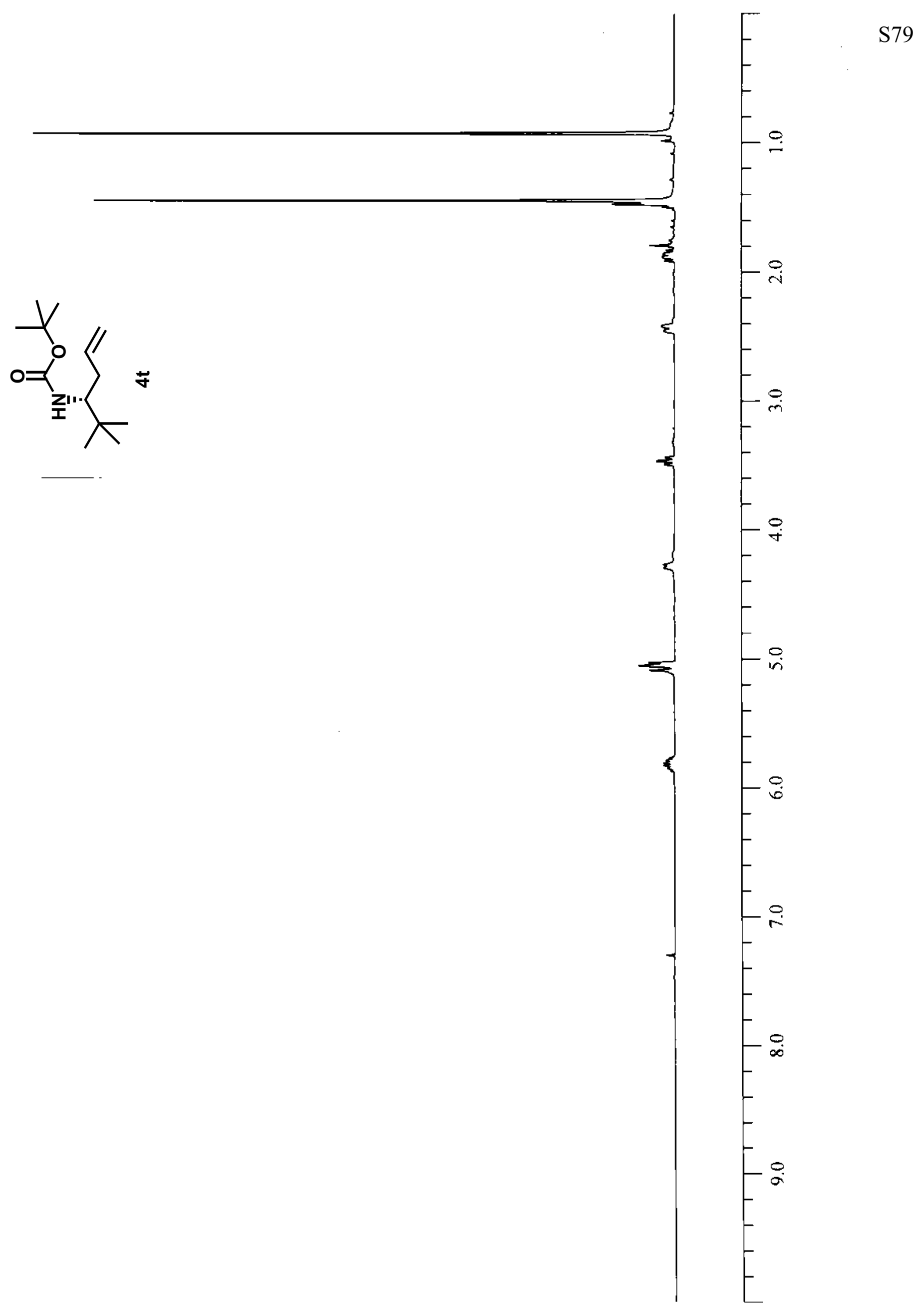




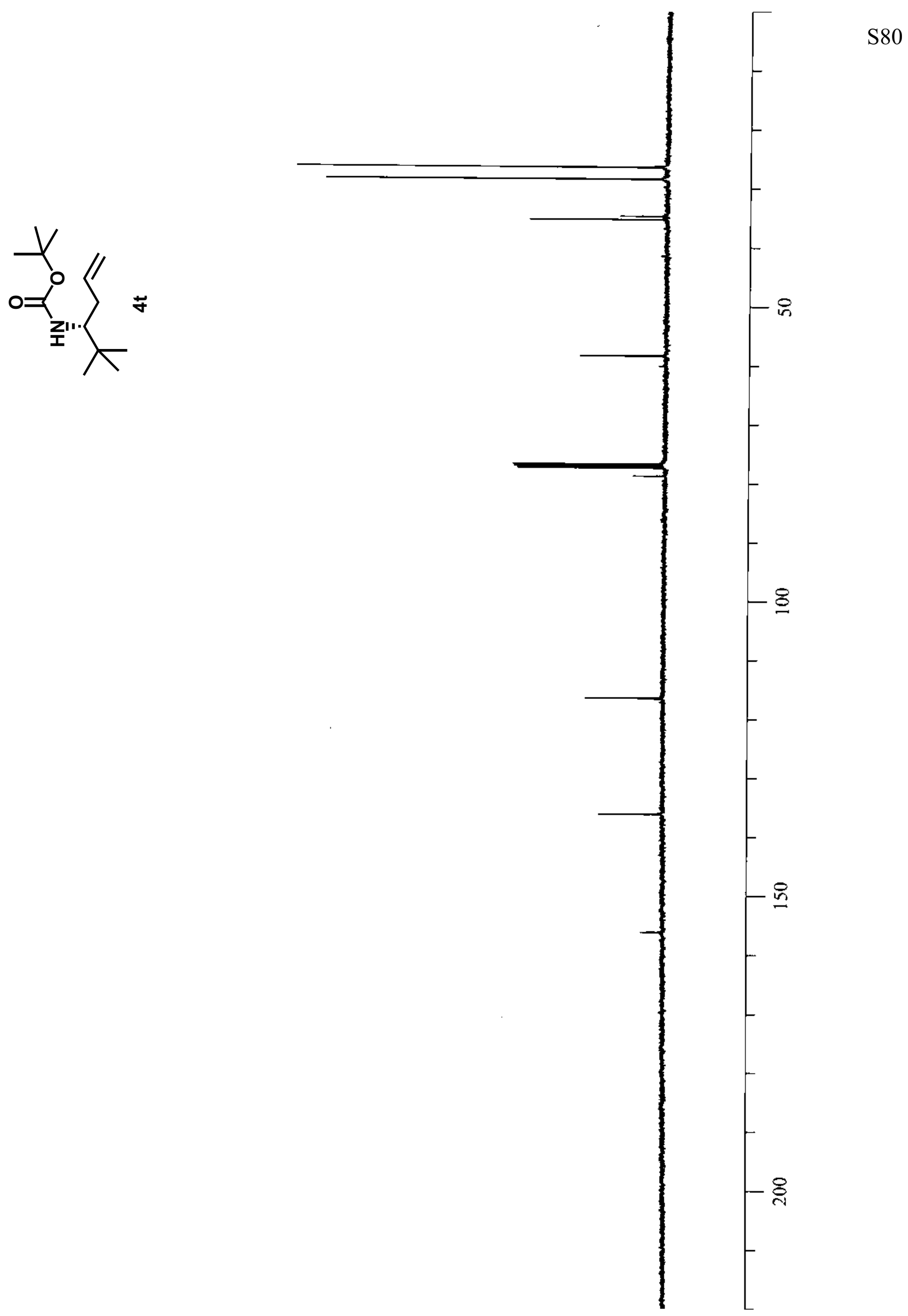




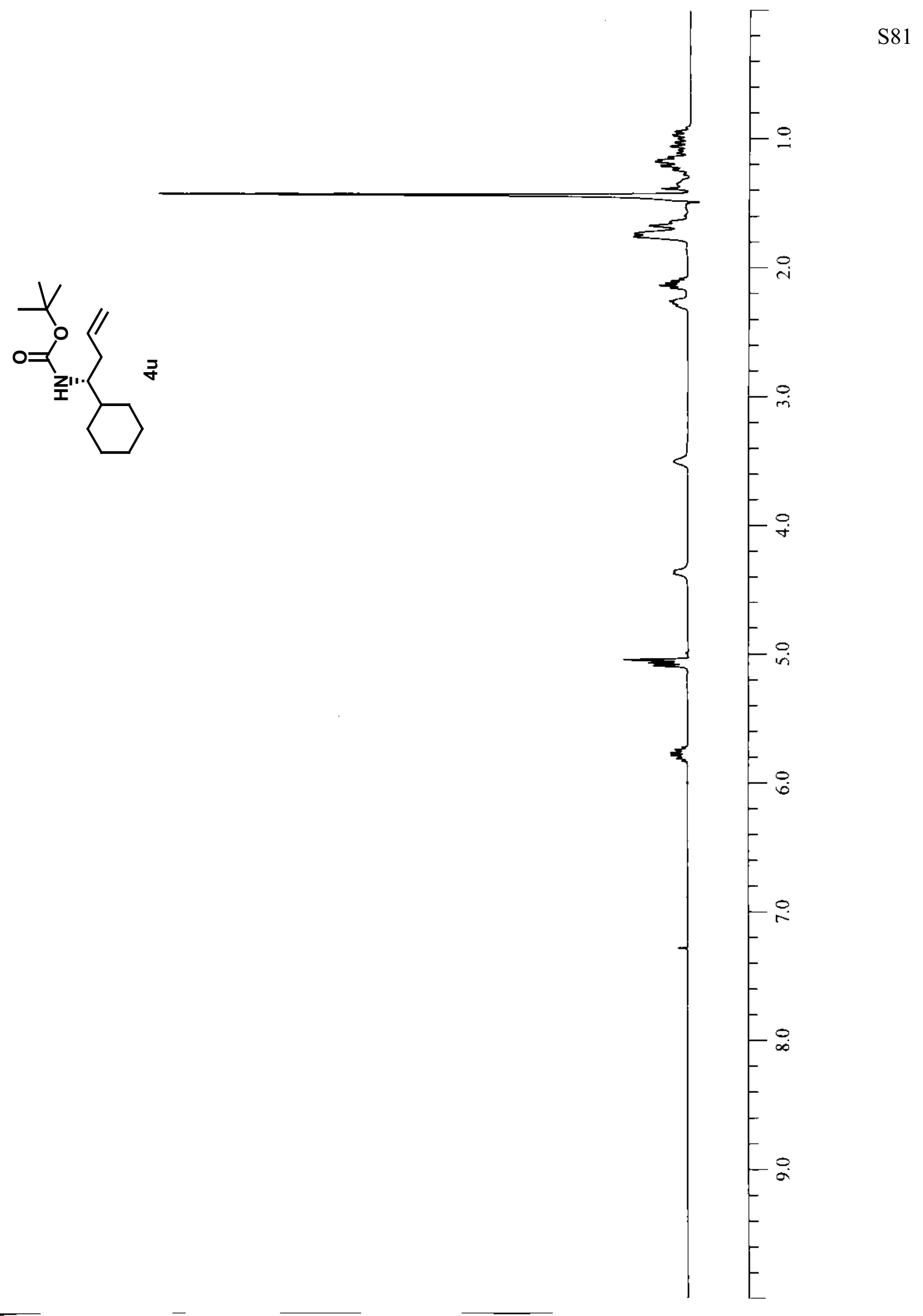



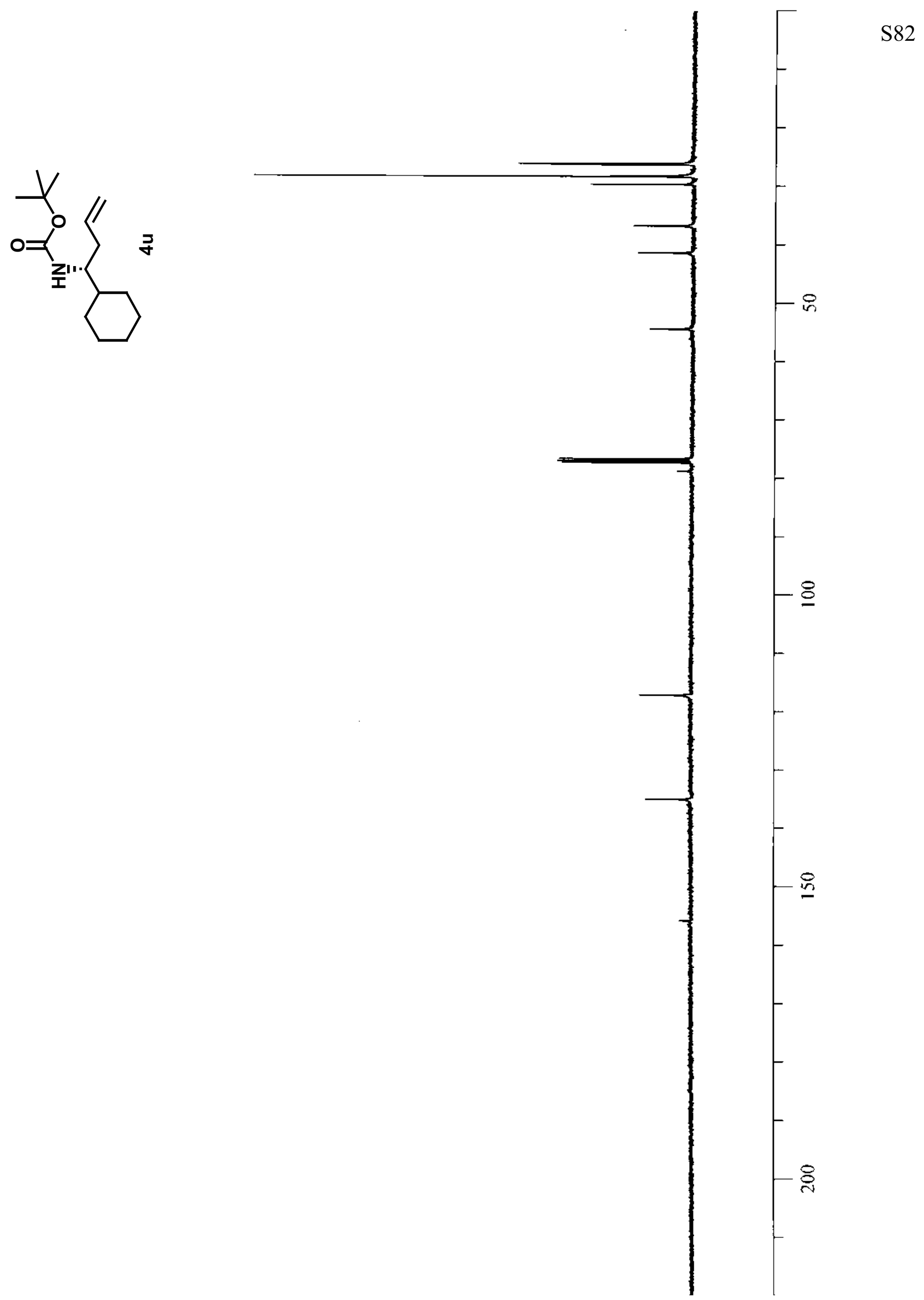


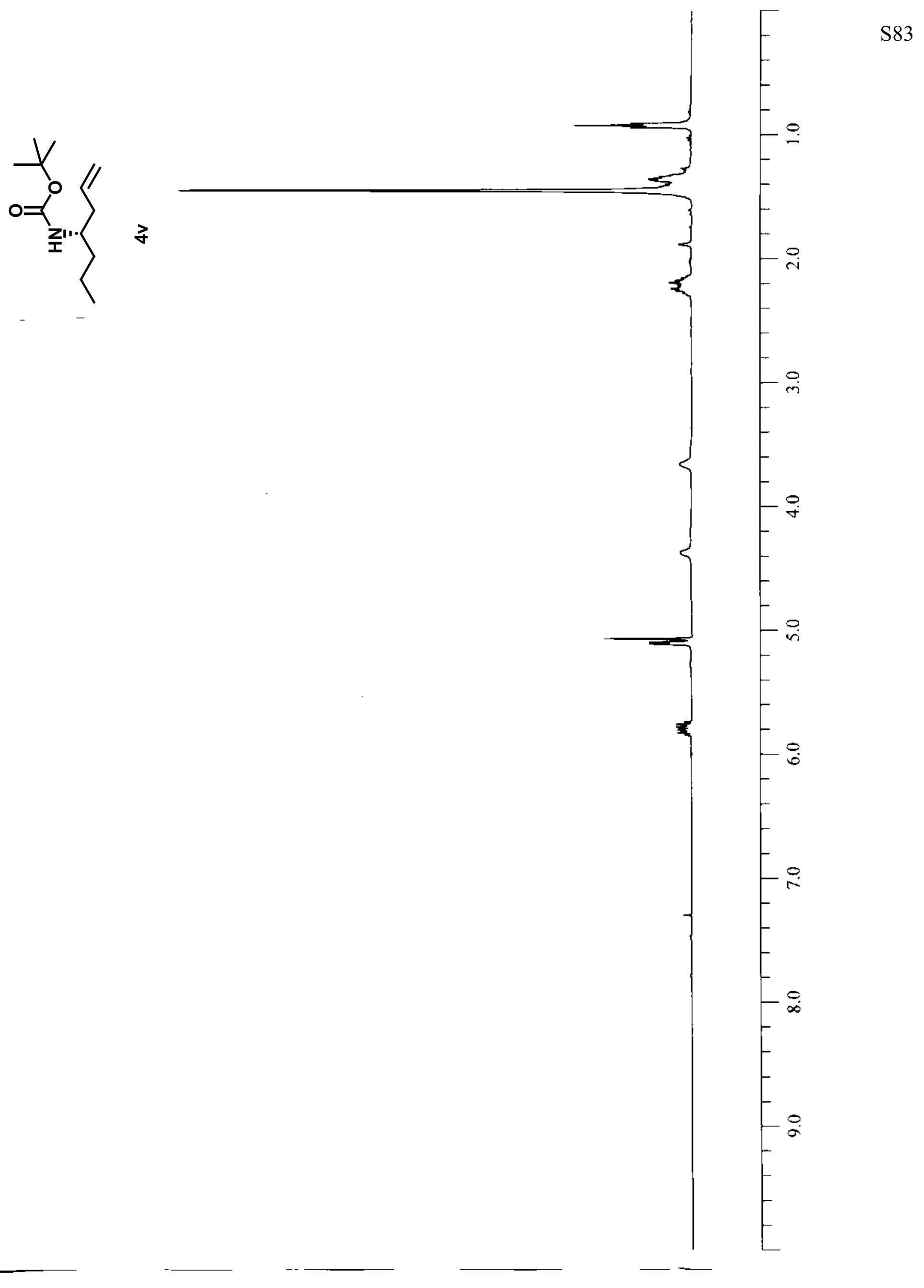




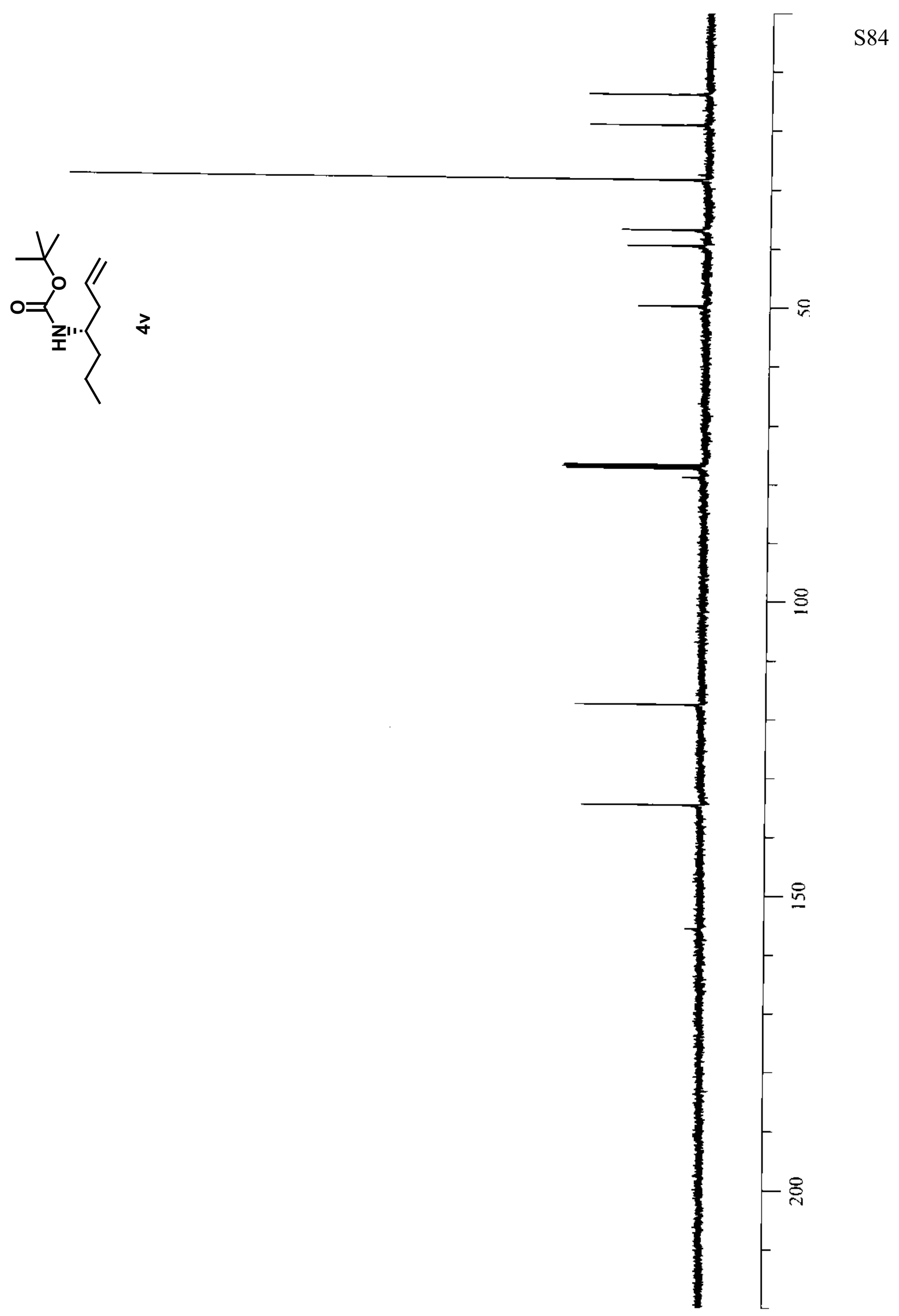




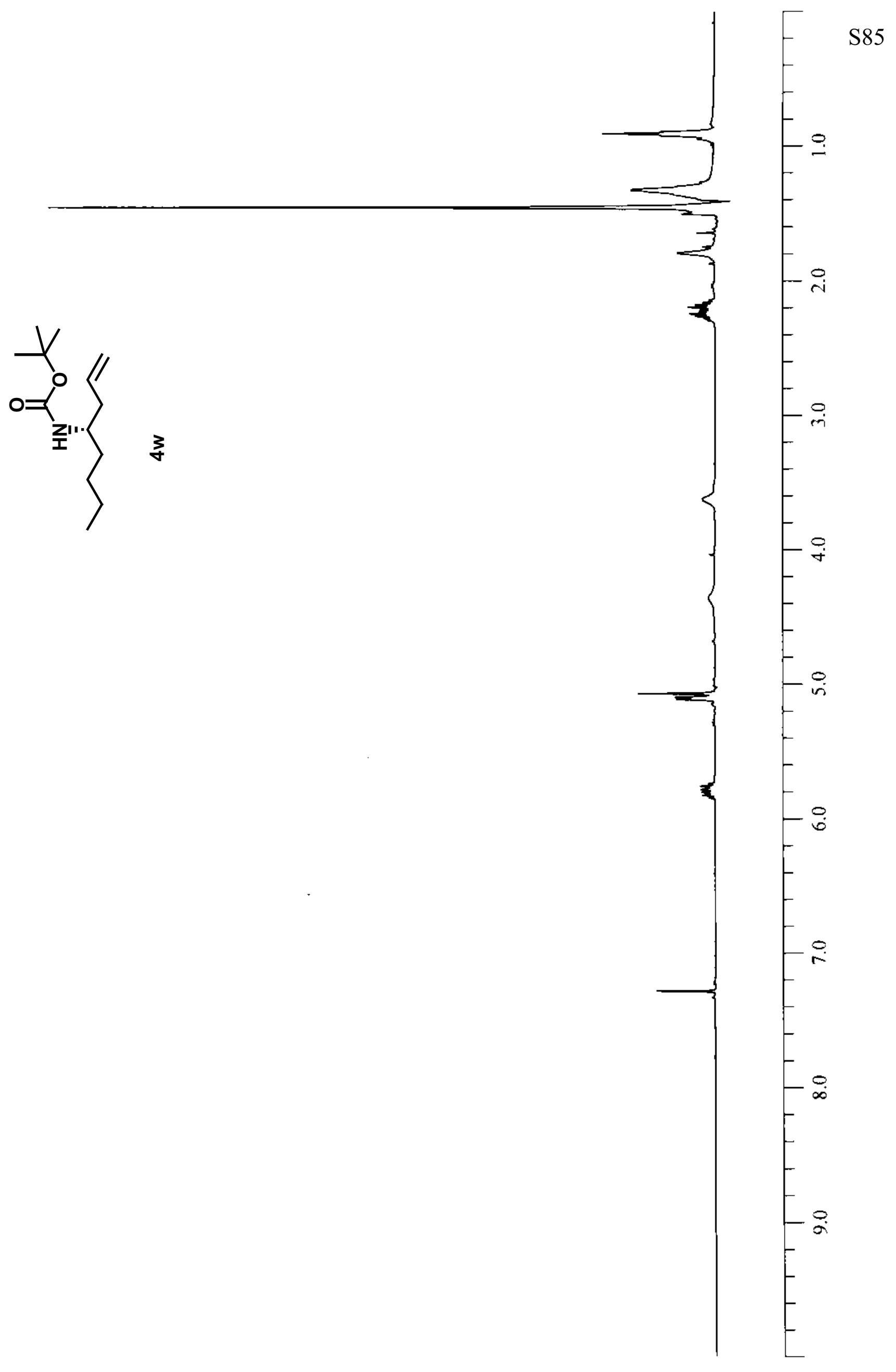




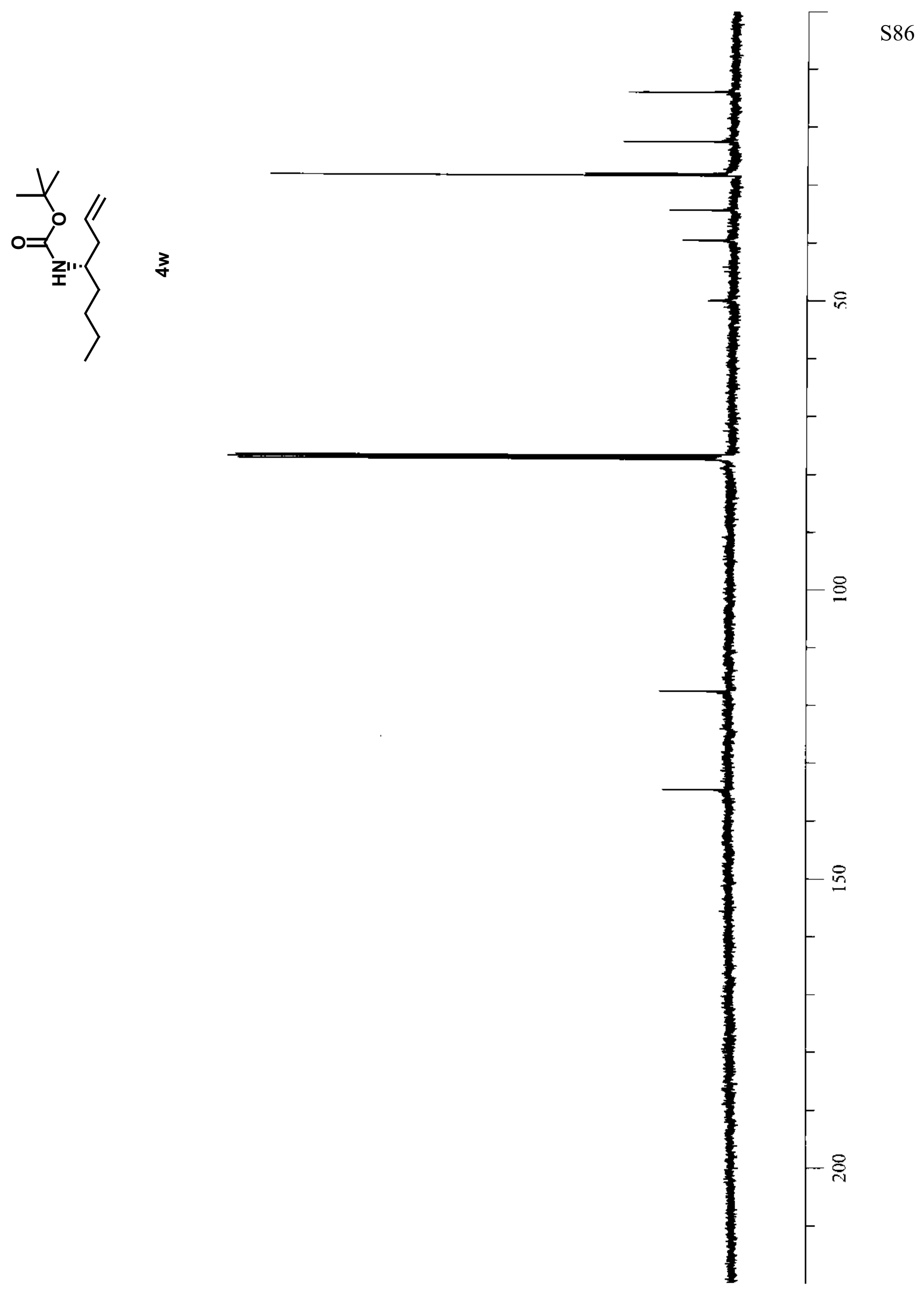




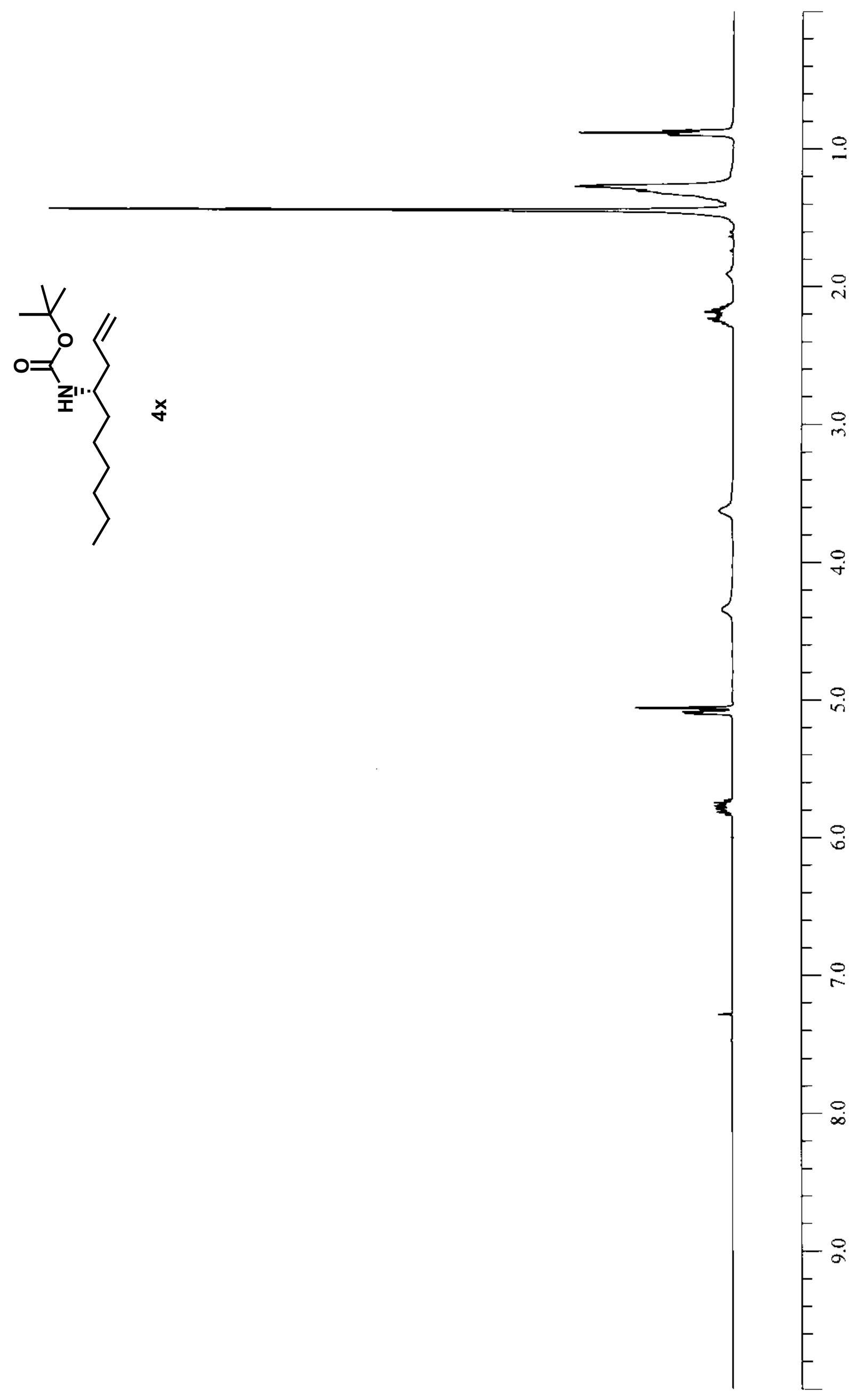




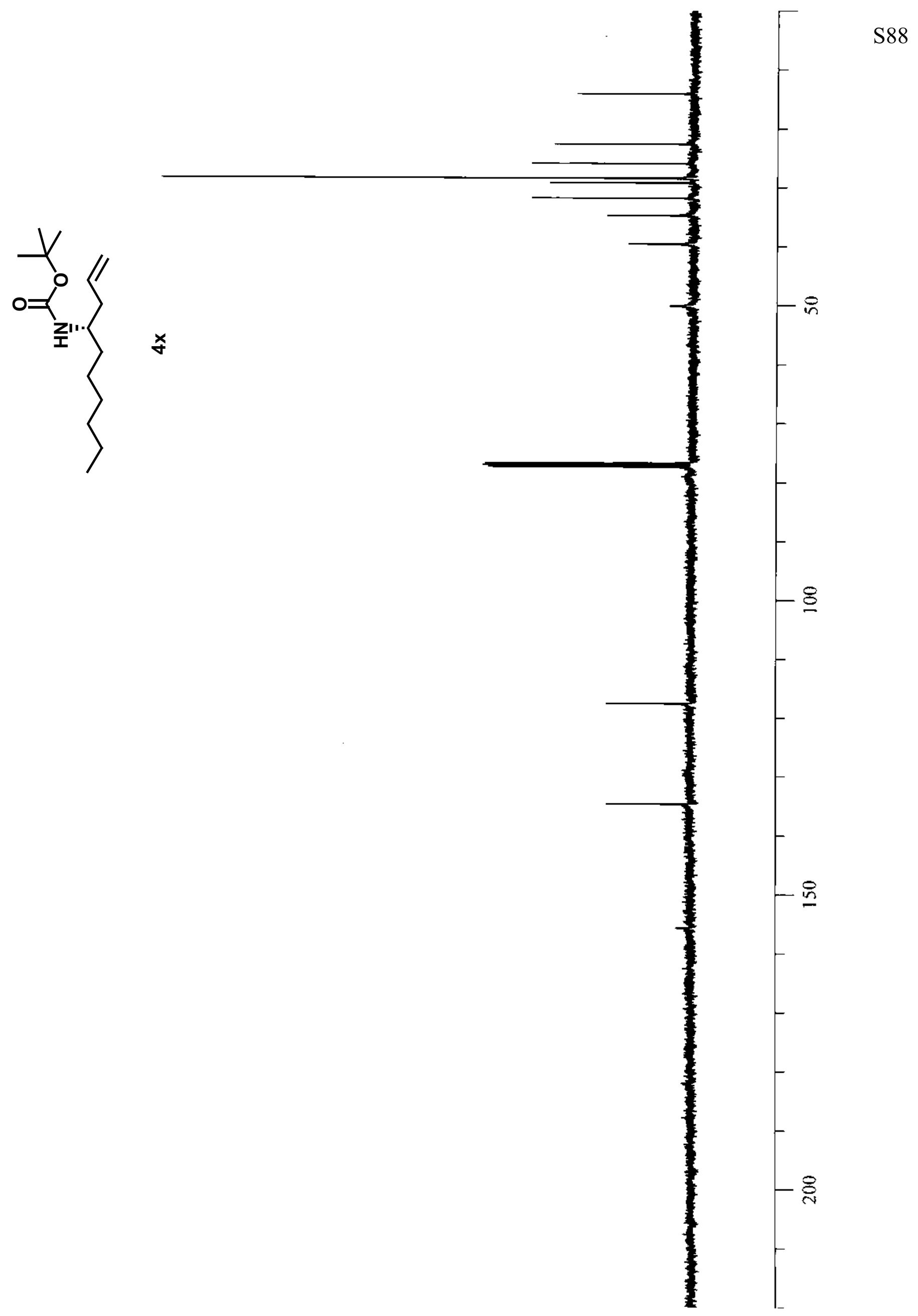




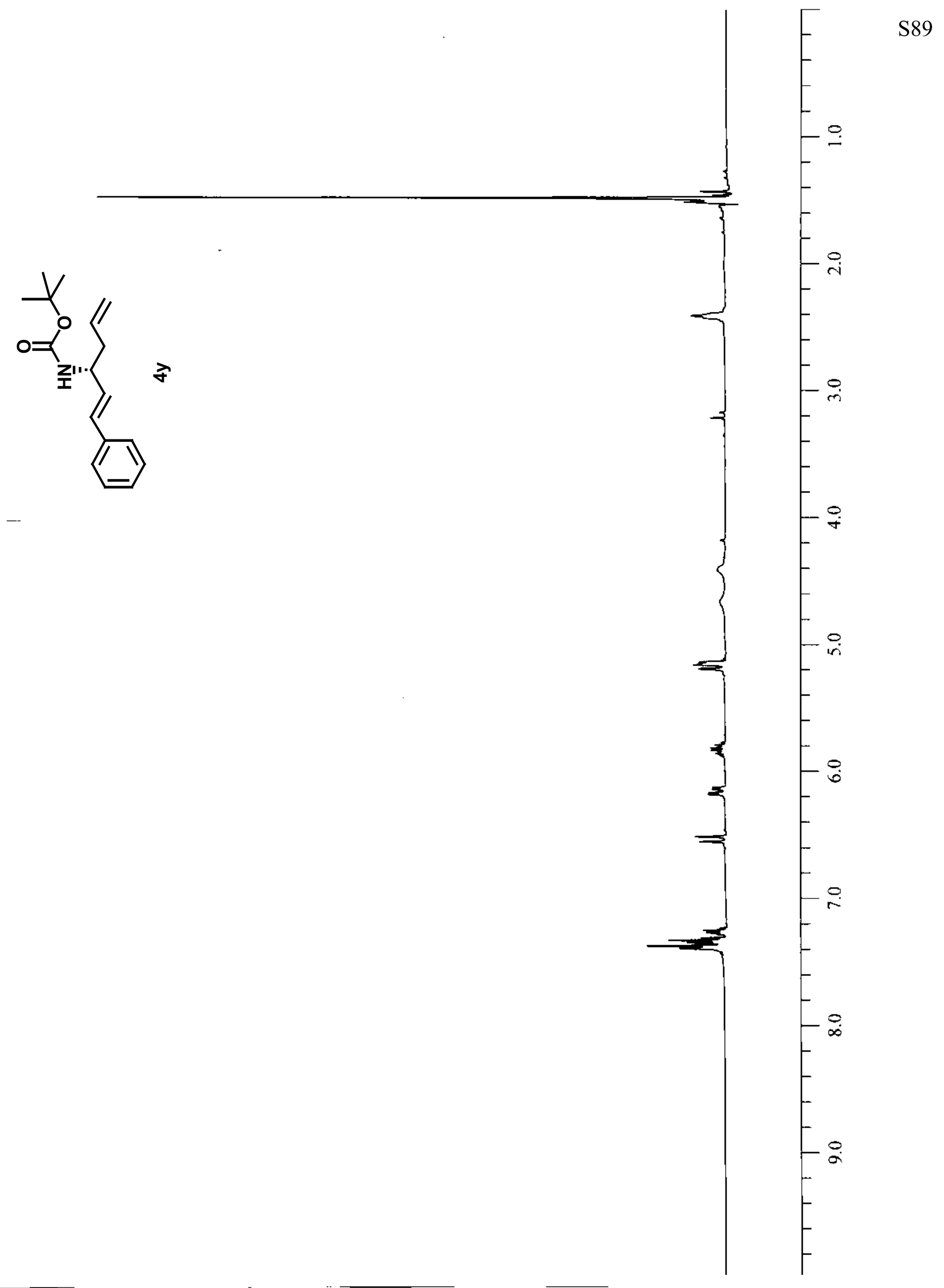




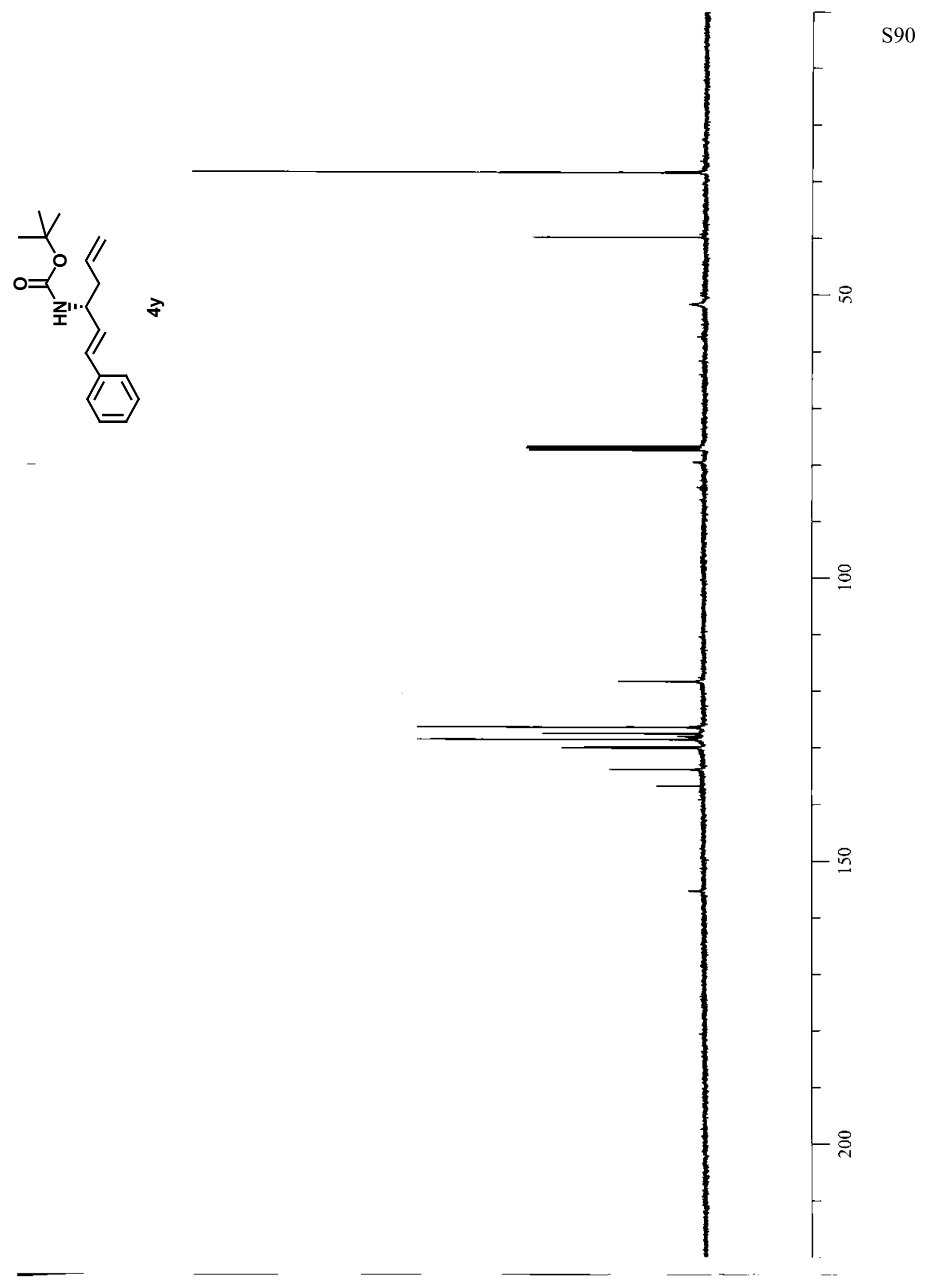




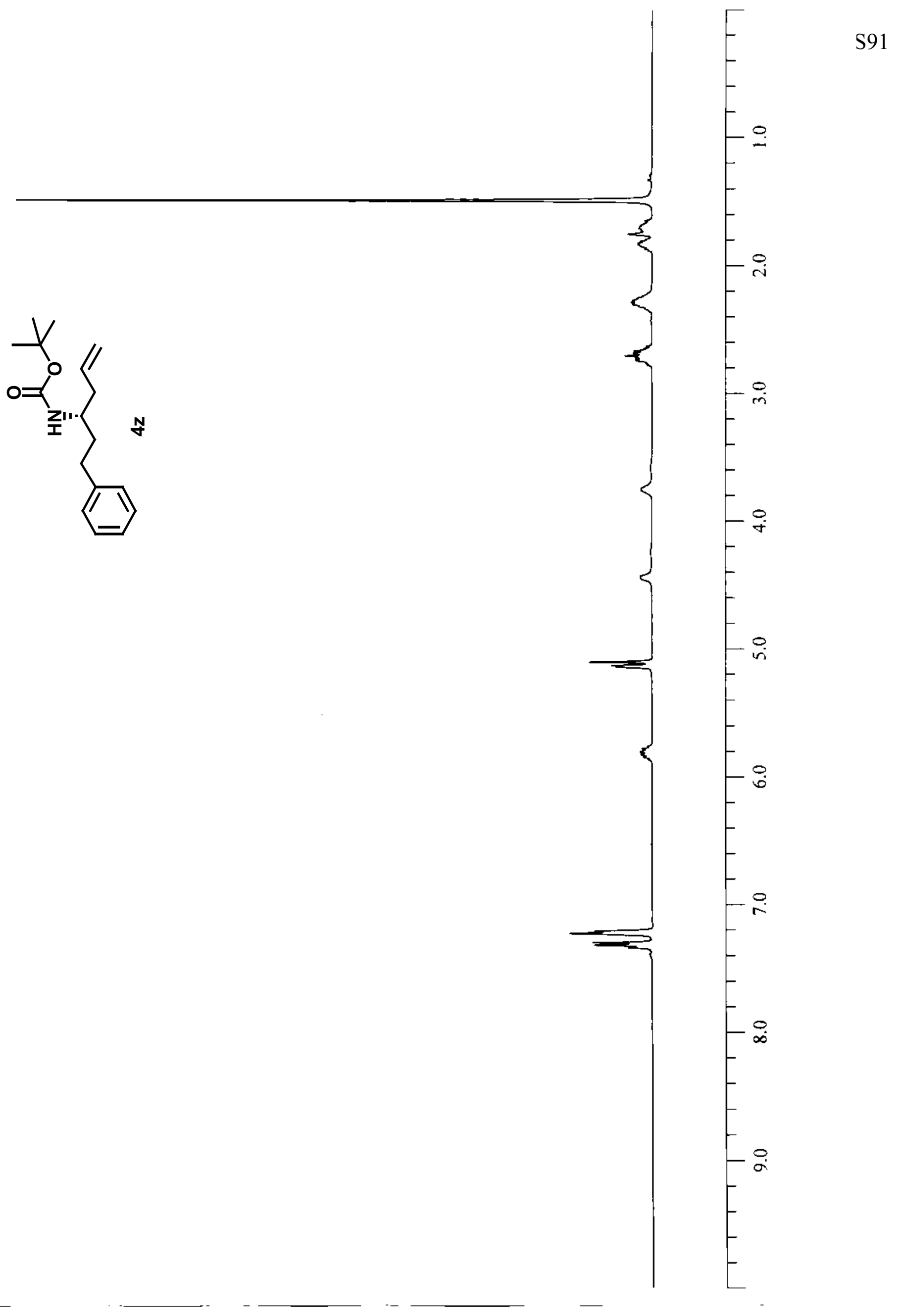




$$
y
$$

